\title{
Raman spectroscopy-based analysis of cartilage composition with applications in finite element modeling \\ By
}

\section{Daniel Ralph Mason, B.Eng.}

\begin{abstract}
A thesis submitted to the Faculty of Graduate and Postdoctoral Affairs in partial fulfillment of the requirements for the degree of
\end{abstract}

\section{Master of Applied Science \\ in}

Mechanical Engineering

Carleton University

Ottawa, Ontario

(C2020, Daniel Mason 


\section{Abstract}

Articular cartilage possesses unique material properties due to a complex depth-dependent composition of sub-components. Raman spectroscopy has proven valuable in quantifying this composition through cartilage cross-sections. However, cross-sectioning requires tissue destruction and is not practical insitu. In this thesis, Raman spectroscopy-based multivariate curve resolution was employed in porcine cartilage samples $(n=12)$ to measure collagen II, glycosaminoglycan, and water distributions throughthe-surface and in cross-sections. These data were then used to create depth-dependent material property finite element models of cartilage, optimized to match experimental results. Through-thesurface Raman measurements could predict composition distributions up to a depth of approximately $0.5 \mathrm{~mm}$. Depth-dependent FE models averaged an $18 \%$ reduction in error for predicted reaction force compared to simplified homogeneous distribution models. Use of a fructose-based optical clearing agent was found ineffective in homogenizing scattering. This measurement technique could be applicable for non-destructively modeling the evolution of joint diseases such as osteoarthritis. 


\section{Acknowledgements}

I would like to firstly thank Dr. Andrew Speirs and Dr. Sangeeta Murugkar for their assistance and mentorship through the development of this thesis. Their expertise and guidance were invaluable, and provided me with a solid foundation in academic research.

I would like to thank Harry Allen and Connor McNairn for their assistance with processing of Raman data, and operation of spectrometers.

I would also like to thank my parents Anne-Marie and Ralph Mason, and my partner Dayna Adriaansen for their continued support over the course of this degree.

Finally, I would like to thank the entire department of Mechanical \& Aerospace Engineering at Carleton University for the educational experience I was privileged to obtain. 


\section{Table of Contents}

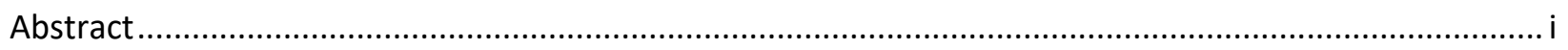

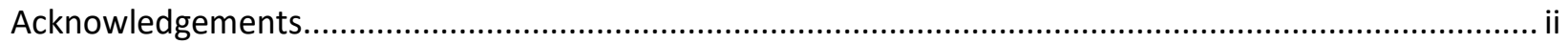

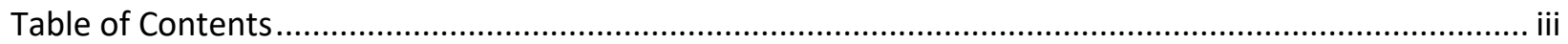

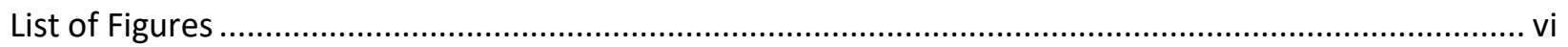

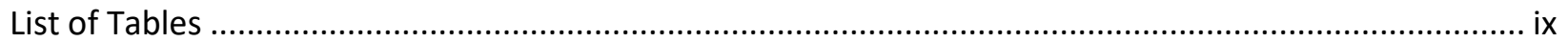

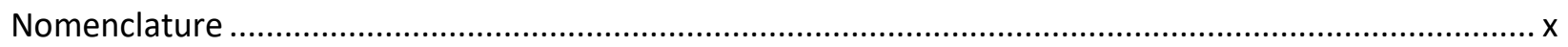

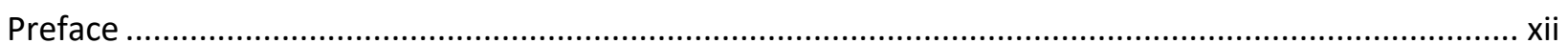

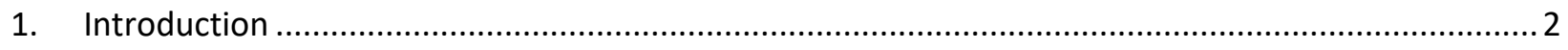

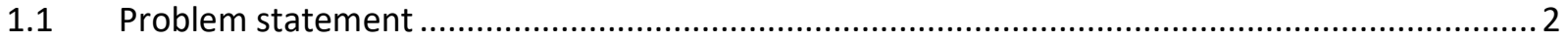

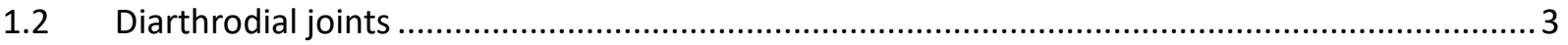

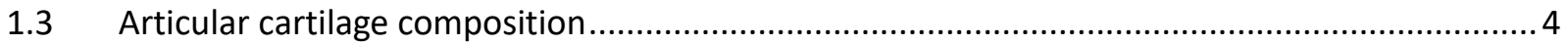

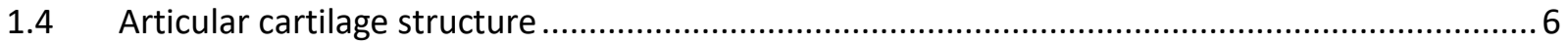

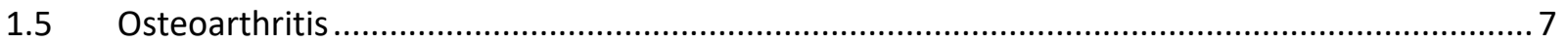

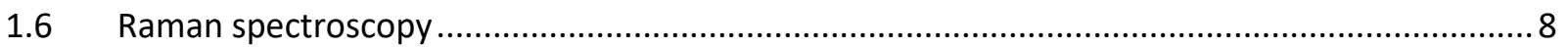

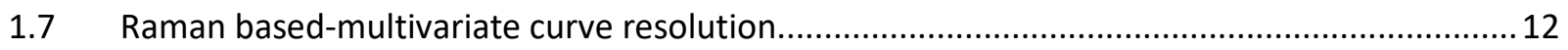

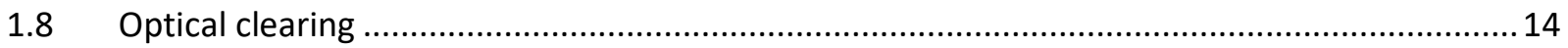

1.9 Cartilage Modeling with Finite Element Analysis ................................................................ 15

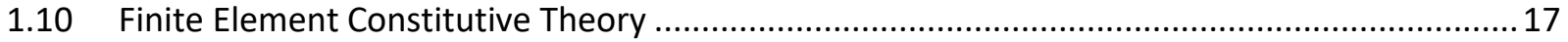

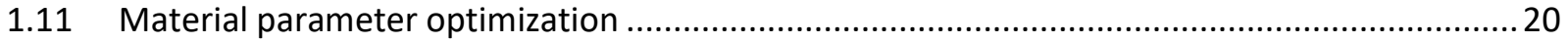

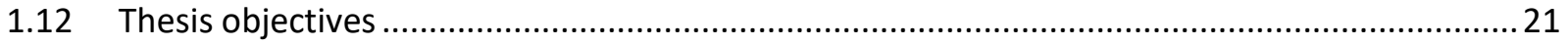

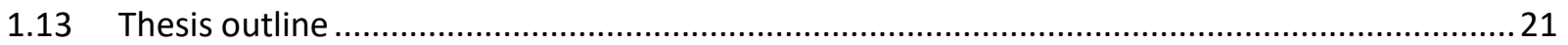

2. Measurement of cartilage sub-component distributions through the surface by Raman

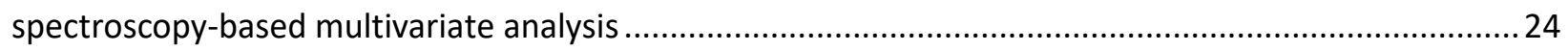

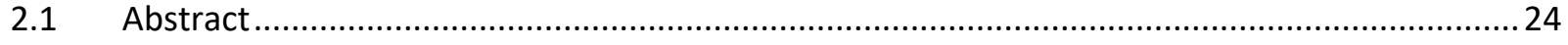

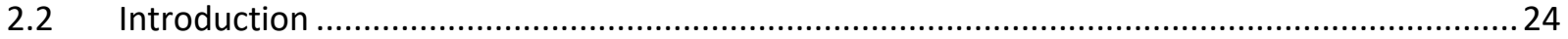

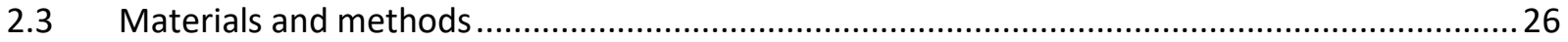

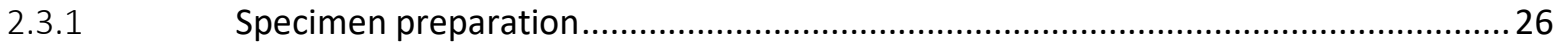

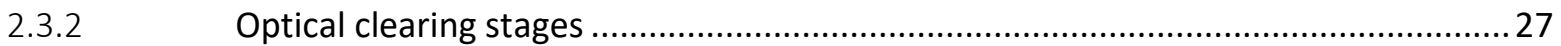

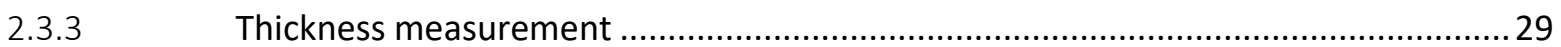

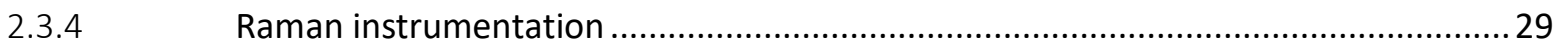

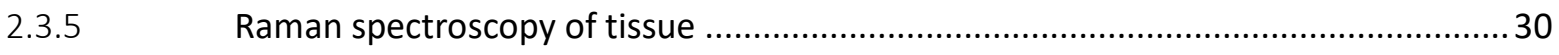




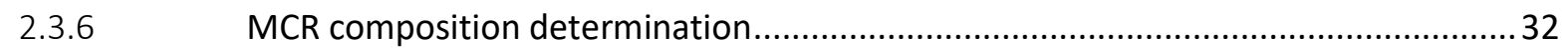

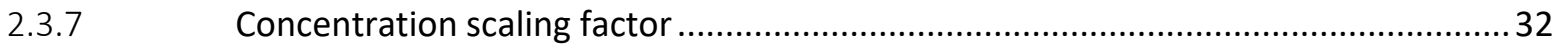

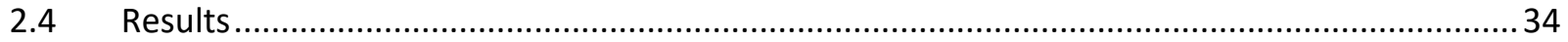

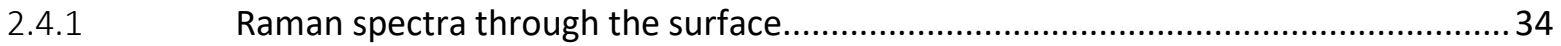

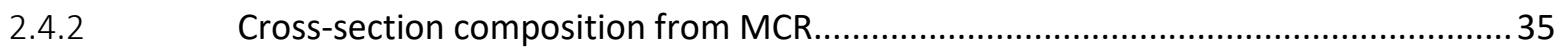

Composition through-the-surface and clearing effects............................................36

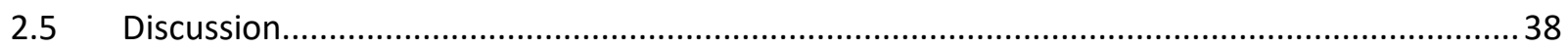

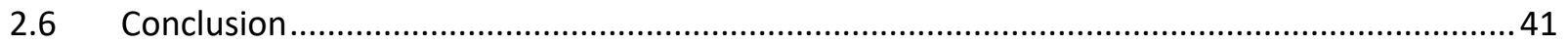

3. Raman spectroscopy-based MCR for applying depth-dependent material properties to articular

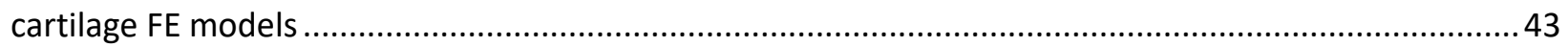

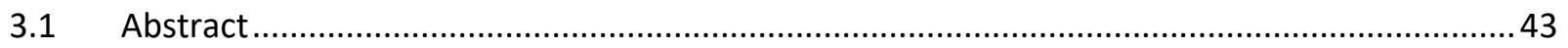

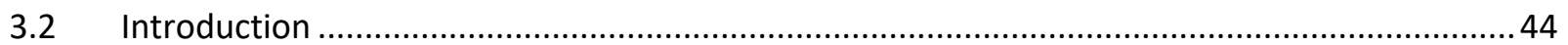

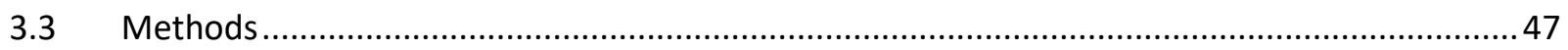

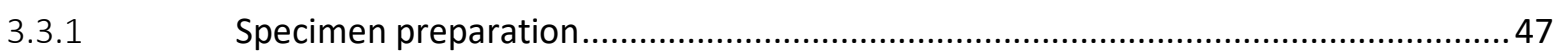

3.3.2 Mechanical loading and testing regime ........................................................... 47

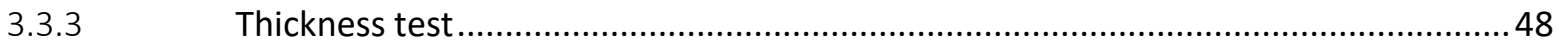

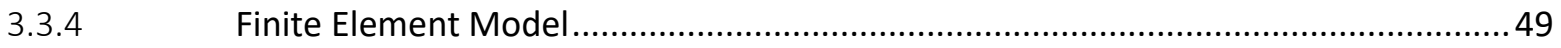

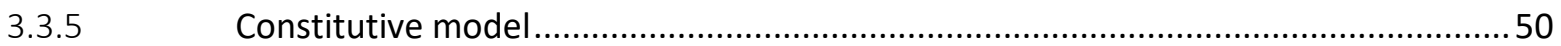

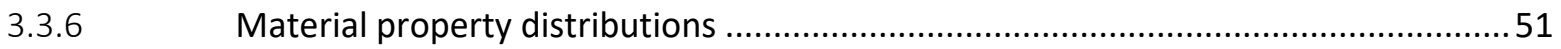

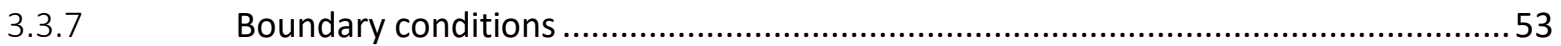

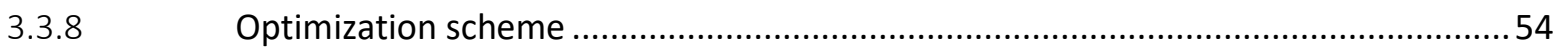

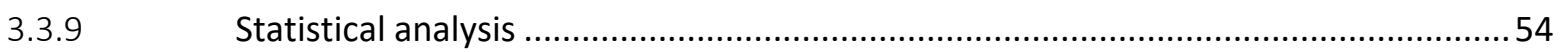

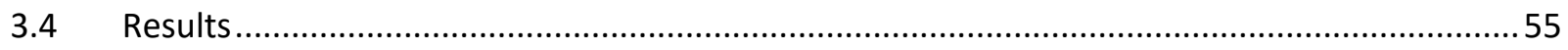

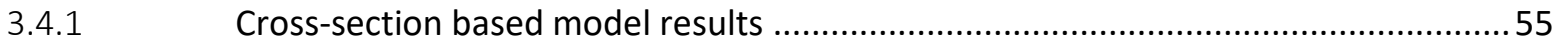

3.4.2 Through-the-surface based model results ..........................................................56

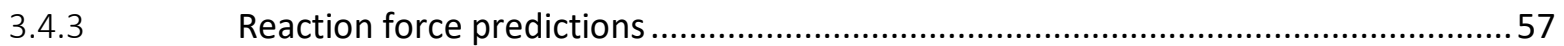

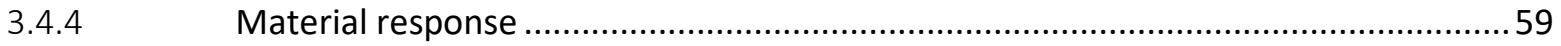

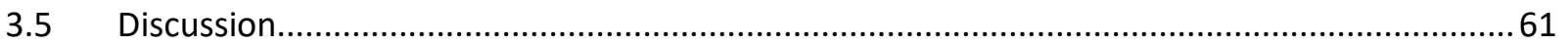

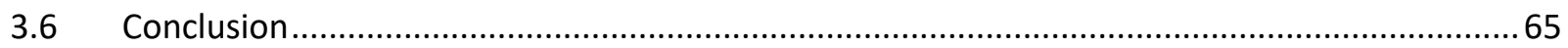

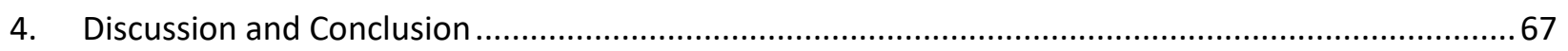

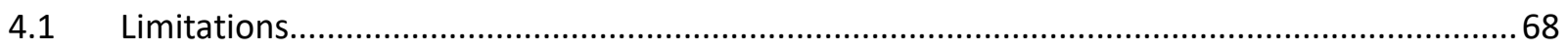

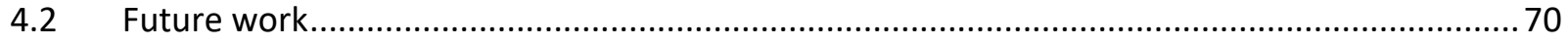

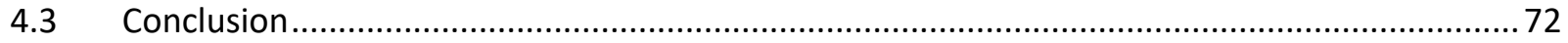




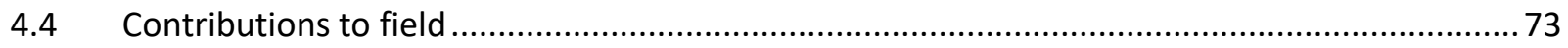

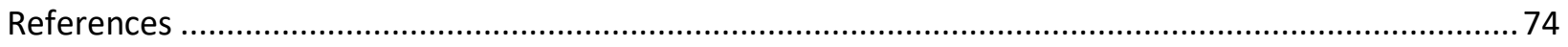

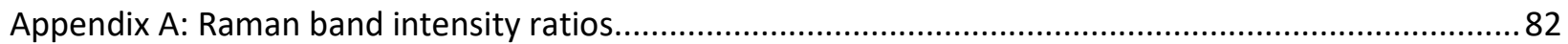

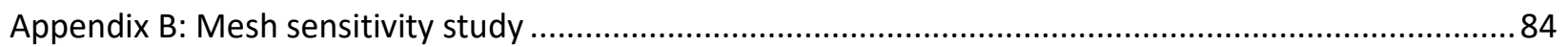

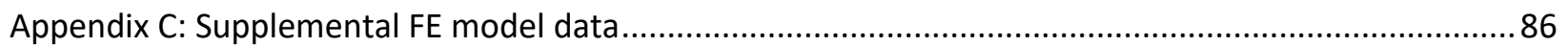

Appendix D: Depth-dependent permeability FE models ....................................................................91

Appendix E: Raman spectra processing scripts (pseudo code)........................................................ 94

E.1 Function for removing cosmic rays from spectra ............................................................ 95

E.2 Function for removing fluorescent background from spectra .............................................96

E.3 Script for processing Raman spectra from cartilage .............................................................96

Appendix F: FEBio input files \& DataMap alterations (pseudo code) ....................................................98

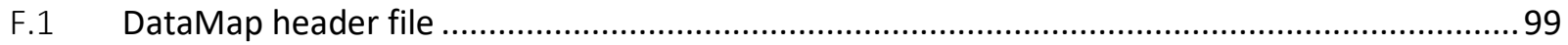

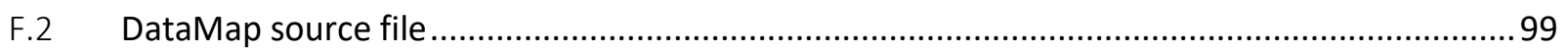

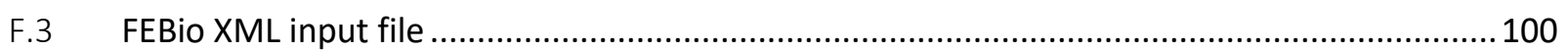

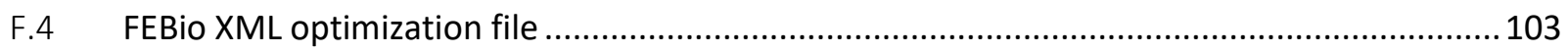




\section{List of Figures}

Figure 1.1: Diagram of diarthrodial joint with labeled components. Adapted with permission from D. Wilkin, and J. Brainard, "Human Biology",CK-12 Foundation, Copyright(2015).

Figure 1.2: (a) Articular cartilage ECM sub-components interacting within the tissue. (b) Proteoglycan structure and scale. Reprinted from Biomaterials, 13(2), V.C. Mow, A. Ratcliffe, A. R. Poole, "Cartilage and diarthrodial joints as paradigms for hierarchical materials and structures", pg. 74-75, Copyright (1992), with permission from Elsevier.

Figure 1.3: Depth dependent structure and composition of articular cartilage. Reprinted from Phys Ther Sport, 16(4), Brody L.T., "Knee osteoarthritis: Clinical connections to articular cartilage structure and function", pg. 302, Copyright (2015), with permission from Elsevier.

Figure 1.4: Molecular energy level diagram illustrating the difference between Rayleigh and Raman scattering $\left(\mathrm{E}_{0}=\right.$ electronic energy state, $\mathrm{E}=$ scattering photon energy, $\mathrm{v}=$ vibrational energy state, $v_{m}=$ shift in scattering frequency associated with a change in vibrational energy levels, $v_{0}=$ incident electric field vibrational frequency, $\mathrm{h}=$ Planck's constant).

Figure 1.5: Typical loading configurations used for testing of cartilage. Adapted from J. Biomech, 34(4), M. DiSilvestro and J. Suh, "A cross-validation of the biphasic poroviscoelastic model of articular cartilage in unconfined compression, indentation, and confined compression", pg. 521, Copyright (2001), with permission from Elsevier.

Figure 2.1: (a) Specimen setup for Raman measurements through-the-surface of cartilage. A wire was placed on the bearing surface parallel to the diameter (within $200 \mu \mathrm{m}$ of measurement location) to determine the reference height. (b) Raman measurement of cross-sectioned cartilage plug alongside plug schematic designating spectral measurement locations with $S$ and $B$ indicating the bearing surface and bone respectively. A wire was placed perpendicular to the bearing surface to determine the reference height. (c) Timeline of optical clearing stages. Images (a) and (b) are repeated in (c).............28

Figure 2.2: Raman spectrometer schematic for measurement of immersed cartilage samples. The specifications of filters, and the CCD camera used, are further detailed in a report by Hansson et al.[76].

Figure 2.3: (a) Raw preclear Raman spectra through-the-surface with no background removal or normalization. (b) Offset pre-processed preclear spectra for treated sample through-the-surface. (c) Offset pre-processed cross-section spectra for comparison. Red labeled wavenumbers specify peaks most associated with collagen II, blue with GAG, and black for shared or other sub-components. 
Figure 2.4: Reference vs. MCR deconvolved sub-component spectra for control sample through crosssection for (a) Water, (b) GAG, (c) Collagen II. Control and treated sample sub-component concentrations based on normalized MCR distribution curves through the cross-section for (d) Water, (e) GAG, (f) Collagen II. Error bars represent the $95 \%$ C.I. Depth measurements within a $10 \%$ thickness range were averaged into one depth based on negligible change in properties. 36

Figure 2.5: MCR deconvolved ratio of cartilage sub-component contributions to the sum of the main three molecules for (a) Water - Treated Samples, (b) Water - Control Samples, (c) GAG - Treated Samples, (d) GAG - Control Samples, (e) Collagen II - Treated Samples, and (f) Collagen II - Control Samples. Error bars represent the $95 \%$ C.I. Depth measurements within a 10\% thickness range were averaged into one depth based on negligible change in properties.

Figure 2.6: (a) Reference $40 \%$ FRUIT OCA Raman spectrum alongside cartilage spectrum at $100 \mu \mathrm{m}$ through-the-surface, (b) MCR deconvolved sub-components from postclear stage of a treated sample. Labeled wavenumbers represent OCA-related peaks.

Figure 3.1: (a) Cartilage sample undergoing thickness test using custom-built device. (b) Typical reaction force plot for thickness test where (+) indicates tissue/bone intersection chosen as thickness value. .... 49

Figure 3.2: Finite element wedge model and boundary constraints used for stress-relaxation indentation testing of articular cartilage. The boundary conditions applied to the wedge reproduce the axisymmetric nature of the test. Yellow material represents the impermeable indenter, white material represents the BPVE cartilage material.

Figure 3.3: Unscaled material property distributions mapped to FE model mesh for an individual control sample. The absolute material parameters applied to the model were scaled by their respective values obtained by optimization as explained in the text.

Figure 3.4: Recovered stage through-the-surface MCR prediction of GAG content divided by the equivalent cross-section prediction for treated and control samples alongside regression lines. Error bars represent the $95 \%$ C.I. for the sample distribution. Depth measurements within a $10 \%$ thickness range were averaged into one depth based on negligible change in properties.

Figure 3.5: (a) Reaction force plot for individual treated sample over the duration of the indentation test. (b) Reaction forces around the end of the ramp displacement showing a comparison of the peak experimental and simulated reaction forces. (c) Comparison of reaction forces near the end of the transient phase of the indentation test. .58 
Figure 3.6: (a) First Principal stress ( $\mathrm{MPa}$ ) at peak reaction force time for the depth-dependent and homogeneous models in a treated sample. Higher stresses were concentrated near the surface in the depth-dependent model compared to the homogeneous model. (b) First Principal strain at peak reaction force time for the depth-dependent and homogeneous models in a treated sample. (c) Effective fluid pressure at peak reaction force time for the depth-dependent and homogeneous models in a treated sample. Higher fluid pressures were observed in the depth-dependent model compared to the homogeneous model.

Figure 3.7: Percent fluid load support of the stress-relaxation test for a single treated sample.

Figure A.1: Difference in intensity ratio of collagen peaks between preclear and recovered stages for treated and control samples (preclear ratio - recovered ratio). Collagen peaks ratios are related to collagen stability as described in Section 1.6.

Figure B.1: Mesh sensitivity study for FE models quantifying the change in percent difference of the maximum effective stress observed in continually refined meshes. .85

Figure D.1: Alterations to the cartilage mesh made to accommodate material property surface-tosurface maps in FEBioStudio 1.0.0. Tetrahedral element deletions are highlighted by the red square....92 


\section{List of Tables}

Table 1.1: Tentatively assigned Raman bands with molecular vibration modes in articular cartilage based on past studies $[16,21,34,39]$.

Table 3.1: Material parameters for FE models determined from optimizations ( $D D=$ Depth-dependent model, $\mathrm{H}=$ Homogeneous model, SVF = Solid volume fraction, $\mathrm{SZ}$ = superficial zone, $\mathrm{MZ}$ = middle zone, DZ = Deep zone). Ranges represent the $95 \%$ confidence interval. All parameters listed were constrained to be positive.

Table C.1: Control sample FE model properties ( $D D=$ depth-dependent, $\mathrm{H}=$ homogeneous, SVF = solid volume fraction). Percent difference in objective function values represent DD models subtracted from $\mathrm{H}$ models.

Table C.2: Treated sample FE model properties (DD = depth-dependent, $\mathrm{H}=$ homogeneous, $\mathrm{SVF}=$ solid volume fraction). Percent difference in objective function values represent DD models subtracted from $\mathrm{H}$ models.

Table C.3: Control sample reaction forces in preclear vs. recovered stages (RF = reaction force). Equilibrium RF represents average of last $10 \mathrm{~s}$ of $1800 \mathrm{~s}$ indentation test.

Table C.4: Treated sample reaction forces in preclear vs. recovered stages ( $\mathrm{RF}=$ reaction force). Equilibrium RF represents average of last $10 \mathrm{~s}$ of $1800 \mathrm{~s}$ indentation test. .....

Table C.5: Control sample FE model material response ( $\mathrm{RF}=$ reaction force, $\mathrm{EXP}=$ experimental, $\mathrm{DD}=$ depth-dependent model, $\mathrm{H}$ = homogeneous model, $\mathrm{FLS}=$ fluid load support). Equilibrium RF represents average of last $10 \mathrm{~s}$ of $1800 \mathrm{~s}$ indentation test.

Table C.6: Treated sample FE model material response $(\mathrm{RF}=$ reaction force, $\mathrm{EXP}=$ experimental, $\mathrm{DD}=$ depth-dependent model, $\mathrm{H}$ = homogeneous model, $\mathrm{FLS}=$ fluid load support). Equilibrium RF represents average of last $10 \mathrm{~s}$ of $1800 \mathrm{~s}$ indentation test.

Table D.1: Standard and altered mesh model properties (std = standard depth-dependent model with constant permeability, alt-CP = altered mesh model with constant permeability, alt-DDP = altered mesh model with depth-dependent permeability). 


\section{Nomenclature}

The following is a list of acronyms used within this thesis:

\begin{tabular}{|l|l|}
\hline ALS & Alternating least squares \\
BPVE & Biphasic poroviscoelastic \\
CFD & Continuous fiber distribution \\
ECM & Extracellular matrix \\
FE & Finite element \\
GAG & Glycosaminoglycans \\
LoF & Lack of fit value, measure of un-explained variance in MCR deconvolution \\
MCR & Multivariate curve resolution \\
MRI & Magnetic resonance imaging \\
OA & Osteoarthritis \\
OCA & Optical clearing agent \\
PCM & Pericellular matrix \\
\hline
\end{tabular}

The following is a list of the symbols used in this thesis:

\begin{tabular}{|c|c|c|}
\hline Symbol & Description & SI Units \\
\hline$\alpha$ & Electric polarizability & $\mathrm{C} \cdot \mathrm{m}^{2} / \mathrm{V}$ \\
\hline $\boldsymbol{B}$ & Left Cauchy-Green deformation tensor & Dimensionless \\
\hline$b_{\text {component }}$ & Scaling factor for normalized density value of sub-component & $\mathrm{kg} / \mathrm{m}^{3}$ \\
\hline$b^{f}$ & Body force on fluid phase per unit mass & $\mathrm{N} / \mathrm{kg}$ \\
\hline C & Concentration matrix of sub-component spectra & Dimensionless \\
\hline$\overline{\overline{\boldsymbol{C}}}_{\boldsymbol{f}}$ & Spatial elasticity tensor in fibers & $\mathrm{Pa}$ \\
\hline$\overline{\bar{C}}_{m}^{\prime}$ & Spatial elasticity tensor in ground substance & $\mathrm{Pa}$ \\
\hline$\overline{\bar{C}}_{n}$ & Spatial elasticity tensor & $\mathrm{Pa}$ \\
\hline $\boldsymbol{D}$ & Experimental mixture spectra matrix & Dimensionless \\
\hline$d_{i j}$ & Element of the experimental data matrix & Dimensionless \\
\hline $\boldsymbol{E}$ & Incident electric field & $\mathrm{V} / \mathrm{m}$ \\
\hline $\boldsymbol{e}_{i j}$ & Residual of experimental and deconvolved spectra fit & Dimensionless \\
\hline$\overline{\overline{\boldsymbol{F}}}$ & Deformation gradient tensor & Dimensionless \\
\hline$f$ & Objective function of Levenberg-Marquardt algorithm & $\mathrm{N}^{2}$ \\
\hline $\boldsymbol{H}$ & Step function enforcing fiber contributions only in tension & Dimensionless \\
\hline I & Identity tensor & Dimensionless \\
\hline$I_{1}$ & First invariant of the right Cauchy-Green deformation tensor & Dimensionless \\
\hline$I_{n}$ & Square of the fiber stretch & Dimensionless \\
\hline$J$ & Determinant of the deformation gradient tensor (Jacobian) & Dimensionless \\
\hline $\boldsymbol{k}$ & Hydraulic permeability & $\mathrm{m}^{4} / \mathrm{N} \cdot \mathrm{s}$ \\
\hline$M_{\text {Error }}$ & Matrix of error remaining in deconvolution fit & Dimensionless \\
\hline$n^{r}$ & Reference configuration unit vector along fiber orientation & Dimensionless \\
\hline$\widehat{\boldsymbol{P}}_{\boldsymbol{d}}^{s}$ & Momentum exchange between solid and fluid constituents & $\mathrm{kg} \cdot \mathrm{m} / \mathrm{s}$ \\
\hline$\nabla p$ & Fluid pressure gradient & $\mathrm{Pa} / \mathrm{m}$ \\
\hline$\dot{Q}$ & Nuclear displacement & $\mathrm{m}$ \\
\hline$R^{2}$ & Coefficient of determination & Dimensionless \\
\hline
\end{tabular}




\begin{tabular}{|c|l|l|}
\hline $\boldsymbol{S}$ & Sub-component spectra matrix & Dimensionless \\
$\boldsymbol{t}$ & Time & $\mathrm{S}$ \\
$\overline{\boldsymbol{t}}_{\boldsymbol{n}}$ & Cauchy stress tensor & $\mathrm{Pa}$ \\
$\boldsymbol{w}$ & Fluid flux & $\mathrm{kg} / \mathrm{m}^{2} \cdot \mathrm{s}$ \\
$\boldsymbol{y}_{\boldsymbol{i}}, \boldsymbol{x}_{\boldsymbol{i}}$ & Experimental reaction force data points & $\mathrm{N}$ \\
$\boldsymbol{y}\left(\boldsymbol{x}_{\boldsymbol{i}} ; \boldsymbol{a}\right)$ & Predicted reaction force data points as a function of input parameters & $\mathrm{N}$ \\
$\boldsymbol{\beta}$ & Exponential term in fiber strain energy density function & Dimensionless \\
$\boldsymbol{\lambda}$ & Lamé's first parameter & $\mathrm{Pa}$ \\
$\boldsymbol{\lambda}_{\boldsymbol{i}}$ & Wavelength of incident light & $\mathrm{m}$ \\
$\boldsymbol{\lambda}_{\boldsymbol{s h i f t}}$ & Wavelength of scattered light & $\mathrm{m}$ \\
$\boldsymbol{\mu}$ & Lamé's second parameter & $\mathrm{Pa}$ \\
$\boldsymbol{\mu}_{\boldsymbol{f}}$ & Coefficient of friction & $\mathrm{Dimensionless}$ \\
$\boldsymbol{\mu}_{\boldsymbol{m}}$ & Electric dipole moment & $\mathrm{C} \cdot \mathrm{m}$ \\
$\boldsymbol{\xi}$ & Fiber tensile modulus parameter & $\mathrm{Pa}$ \\
$\boldsymbol{\rho}_{\boldsymbol{c o m p o n e n t}}$ & Normalized density value of sub-component & $\mathrm{Dimensionless}$ \\
$\boldsymbol{\rho}^{\boldsymbol{f}}$ & True fluid density & $\mathrm{kg} / \mathrm{m}^{3}$ \\
$\overline{\overline{\boldsymbol{\sigma}}}_{\boldsymbol{f}}$ & Stress in the continuous fiber distribution & $\mathrm{Pa}$ \\
$\overline{\boldsymbol{\sigma}}_{\boldsymbol{m}}$ & Stress in the ground substance material & $\mathrm{Pa}$ \\
$\boldsymbol{\sigma}_{\boldsymbol{t}}$ & Total stress in the biphasic material & $\mathrm{Pa}$ \\
$\boldsymbol{\phi}$ & Phase of the nuclear mode vibration & $\mathrm{Rad}$ \\
$\boldsymbol{\varphi}_{\boldsymbol{s}}$ & Solid phase porosity & $\mathrm{Dimensionless}$ \\
$\boldsymbol{\Psi}$ & Strain energy density function & $\mathrm{N} / \mathrm{m}^{2}$ \\
$\boldsymbol{\omega}_{\boldsymbol{0}}$ & Vibrational frequency of an incident electric field & $\mathrm{Rad} / \mathrm{s}$ \\
$\boldsymbol{\omega}_{\boldsymbol{m}}$ & Nuclear resonance frequency & $\mathrm{Rad} / \mathrm{s}$ \\
\hline
\end{tabular}




\section{Preface}

This integrated thesis contains two manuscripts, one published and the other in the process of being published to a scientific journal. The first manuscript has been published in the Journal of Biophotonics as:

Mason, D.R., Murugkar, S. and Speirs, A.D. (2020), Measurement of cartilage sub-component distributions through the surface by Raman spectroscopy-based multivariate analysis. J. Biophotonics. Accepted Author Manuscript. doi:10.1002/jbio.202000289

Chapter 2 reflects a version of this published manuscript, adapted for formatting within the thesis and based on examiner recommendations.

The second manuscript is being submitted to the Journal of Osteoarthritis and Cartilage as:

Mason D.R., Murugkar S., Speirs A.D., "Raman spectroscopy-based MCR for applying depth-dependent material properties to articular cartilage FE models".

Chapter 3 reflects the draft prepared for submission of this manuscript, with alterations made to follow formatting within the thesis and based on examiner recommendations. 
Chapter 1:

Introduction and literature review 


\section{Introduction}

\subsection{Problem statement}

Everyday locomotion in humans results in their musculature exerting forces on the surrounding bones. The resulting movement is dependent on bone-to-bone connections to transfer these forces and moments to the desired location. The linkages that make this possible in the majority of the body are diarthrodial, or synovial, joints [1]. Within diarthrodial joints the bearing surfaces enabling this motion are made up of a tissue called articular cartilage [2]. Failure of these joints leads to arthritis [1] which is a term used to describe over 100 joint related diseases [3]. When this joint failure is specifically related to the destruction of articular cartilage, and without inflammation, it is known as osteoarthritis (OA) [4]. $\mathrm{OA}$ is a chronic and debilitating disease which can severely limit the quality of life in those affected [5]. It is also the most common joint disease among adult humans [6], with some studies suggesting up to a third of the adult population show radiological symptoms [7]. Reports from Statistics Canada estimate $10 \%$ of the population above the age of 15 is affected by OA [8]. While inflammation is a major side effect of $O A$, it is not considered a classic inflammatory disease due to the lack of a systemic inflammatory response [9]. The average age of symptom onset in Canadians is approximately 47 years old, with the majority of cases (55\%) being reported in the people above the age of 49 [8]. While OA is reported in numerous joints, the high load bearing knees and hips are more commonly affected $[6,8]$. Based on these statistics alone it is clear that joint diseases in general, and OA specifically, represent a significant burden to the Canadian health care system. Direct costs of OA on the Canadian economy are expected to exceed $\$ 500$ billion by the year 2040 [5]. While its symptoms are well documented, the specific origins of the disease are not well understood. However, its pathogenesis is intimately linked to slowly developing changes in the complex structure and composition of articular cartilage [4]. Research into better understanding the role sub-components play, along with their distributions, in this 
composition is essential to gaining information about the etiology of joint diseases like OA. It also offers key insight into the general functionality of this remarkable tissue.

\subsection{Diarthrodial joints}

Diarthrodial joints, for example shown in Figure 1.1, are a feat of evolutionary biomechanics enabling nearly friction-free $\left(\mu_{\mathrm{f}}=0.005-0.05\right)$ movement for hours a day under loads up to ten times body weight $[1,10]$. The simplified structure of these joints consists of adjacent bone ends surrounded by synovial fluid and encapsulated by a fibrous tissue [11]. The contacting bone ends are covered in hyaline cartilage which acts as the bearing surface for load transmission. Lubrication of these contacting surfaces relies on a complex interaction of tissue and hydrodynamic properties [12]. The non-Newtonian synovial fluid in the joint cavity, produced by the synovium, provides a lubricating film less than $15 \mu \mathrm{m}$ thick [1] for these contacting surfaces. The lubricating properties of this fluid are mainly due to the presence of glycoproteins like hyaluronate and lubricin $[1,10,12,13]$. The synovial fluid also serves as the primary medium of nutrient delivery for the cartilage cells due to their lack of a direct blood supply [14]. This lack of direct blood supply means cartilage is limited in its healing capacity. This also means the tissue must rely on its complex load transfer mechanisms, derived from its composition, to prevent degradation. 


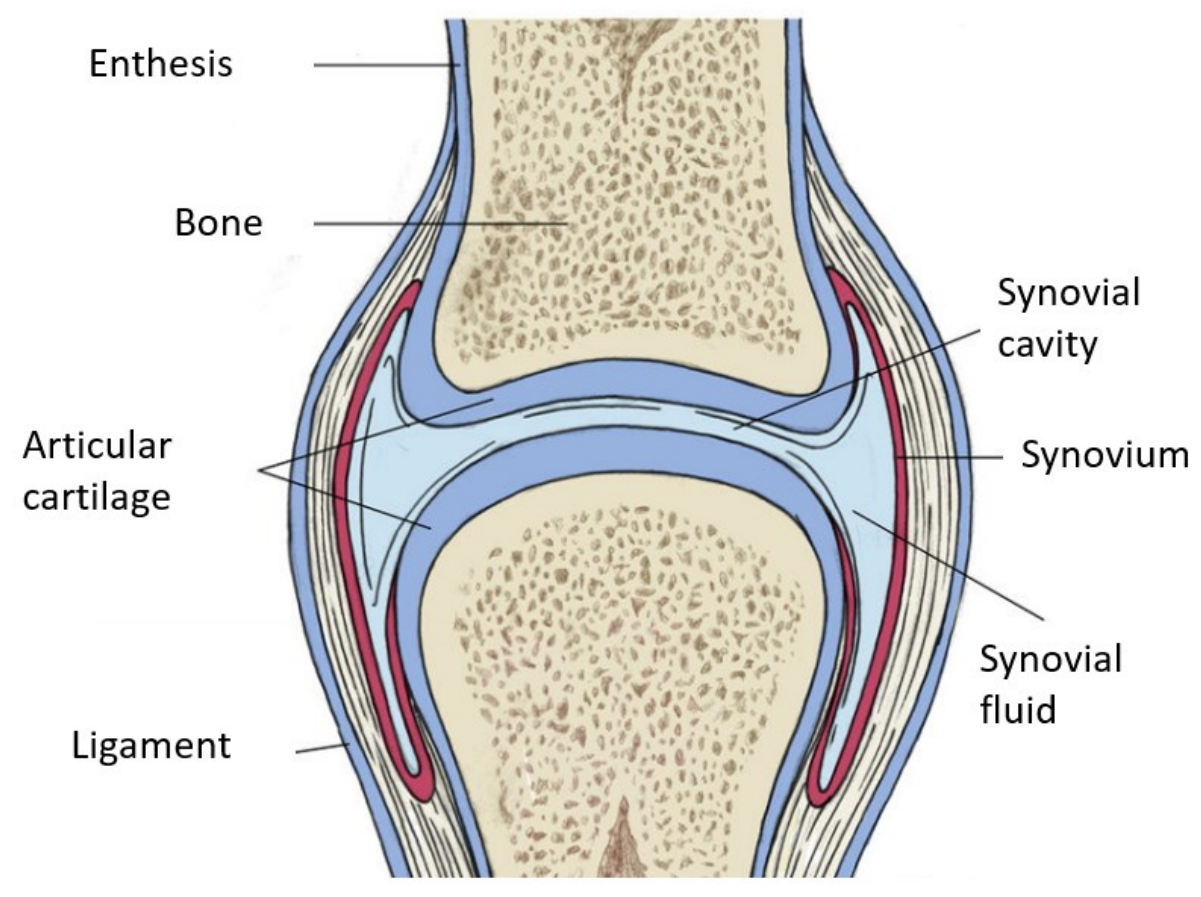

Figure 1.1: Diagram of diarthrodial joint with labeled components. Adapted with permission from D. Wilkin, and J. Brainard, "Human Biology",CK-12 Foundation, Copyright(2015).

\subsection{Articular cartilage composition}

Within the human body there are three forms of cartilage, which are hyaline, elastic, and fibrocartilage [10]. Hyaline cartilage is the predominant form present in diarthrodial joints, where it is referred to as articular cartilage when present on the articulating ends of bones [1]. Articular cartilage is a porous material made up an extra cellular matrix (ECM) filled with interstitial fluid and a multitude of macromolecules. The interstitial fluid phase of articular cartilage accounts for approximately $80 \%$ of the tissue wet weight [15]. The solid structure is predominantly formed from linked collagen II fibrils creating a scaffold, with embedded chondrocyte cells that produce ECM components [2]. The ECM is also comprised of proteoglycans which make up the second largest portion of organic material in the tissue [1] along with traces of different collagen types (V, VI, IX, XI) as well as elastin. Proteoglycans consist of a protein core to which are covalently-bound branches of glycosaminoglycans (GAG) shown in Figure 
1.2(b). These GAG branches consist of highly charged hydrophilic polymer chains, made from disaccharide subunits, which fan out to cover a large area within the ECM pores [16]. Negative charges present on these polymer chains attract the positive ends of bipolar water molecules which impedes flow. This causes the tissue to swell in its attempts to balance the charges present on the GAG side chains and ion concentration gradients in the interstitial fluid. The resulting pressures this swelling induces are known as Donnan osmotic pressures, contributing to the overall compressive stiffness and permeability of cartilage $[17,18]$.

When a load is applied to the tissue surface the ECM begins to deform and the reduction in volume will cause an outflow of interstitial fluid. However, the extremely low tissue permeability (on the order of $\left.10^{-15} \mathrm{~m}^{4} / \mathrm{N} \cdot \mathrm{s}[15]\right)$ results in high fluid pressure. This flow regulation is critical to reducing stress in the ECM as the pressurized fluid typically provides $90 \%$ of the bearing capacity in dynamic loading situations [19]. With the load held constant, the fluid will gradually flow out, reducing the pressure and transferring the load support to the ECM.
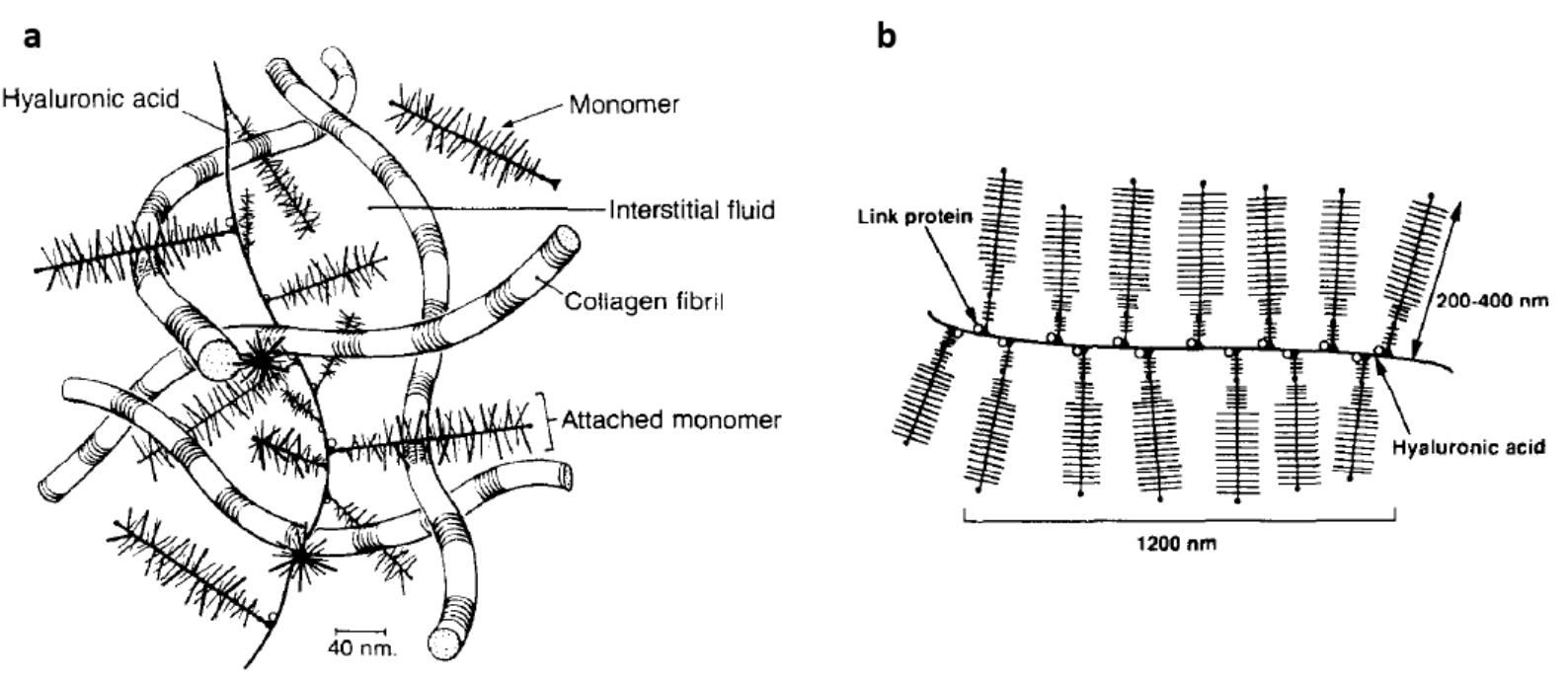

Figure 1.2: (a) Articular cartilage ECM sub-components interacting within the tissue. (b) Proteoglycan structure and scale. Reprinted from Biomaterials, 13(2), V.C. Mow, A. Ratcliffe, A. R. Poole, "Cartilage and diarthrodial joints as paradigms for hierarchical materials and structures", pg. 74-75, Copyright (1992), with permission from Elsevier. 


\subsection{Articular cartilage structure}

The macroscale structure of articular cartilage is traditionally sub-divided into three main zones: The superficial zone, the middle zone, and the deep zone [10]. The superficial zone comprises the top 10$20 \%$ of the tissue thickness. In this zone collagen fibrils are primarily arranged parallel to the articular surface to aid in resisting the shear forces present during joint movement [10]. The next $40-60 \%$ of tissue depth is classified as the middle zone where collagen fibrils are more randomly oriented. The remaining $30-40 \%$ of the tissue makes up the deep zone. In this region collagen fibrils are primarily oriented perpendicular to the articulating surface and insert into the subchondral bone below $[1,2]$. The tissue-bone interface is bridged by a calcified zone, starting at the 'tide mark' illustrated in Figure 1.3. Throughout these zones the proteoglycan content gradually increases with depth [20,21]. Water content reaches its maximum ( $90 \%$ wet mass) near the bearing surface before dropping and maintaining approximately $75 \%$ of the wet mass in the middle and deep zones [22]. The chondrocytes distributed throughout these regions begin with an elliptical shape in the superficial zone and gradually transition to a spherical shape in the deep zone [14]. These chondrocytes embed themselves in the ECM through the less stiff pericellular matrix (PCM) with an approximate thickness of 3-4 $\mu \mathrm{m}$ [23]. As noted by Darling et al. [23] the exact function of the PCM is not entirely known, but it is thought to aid in protection of the chondrocyte as well as transmit mechanical stimuli from the surrounding tissue. 


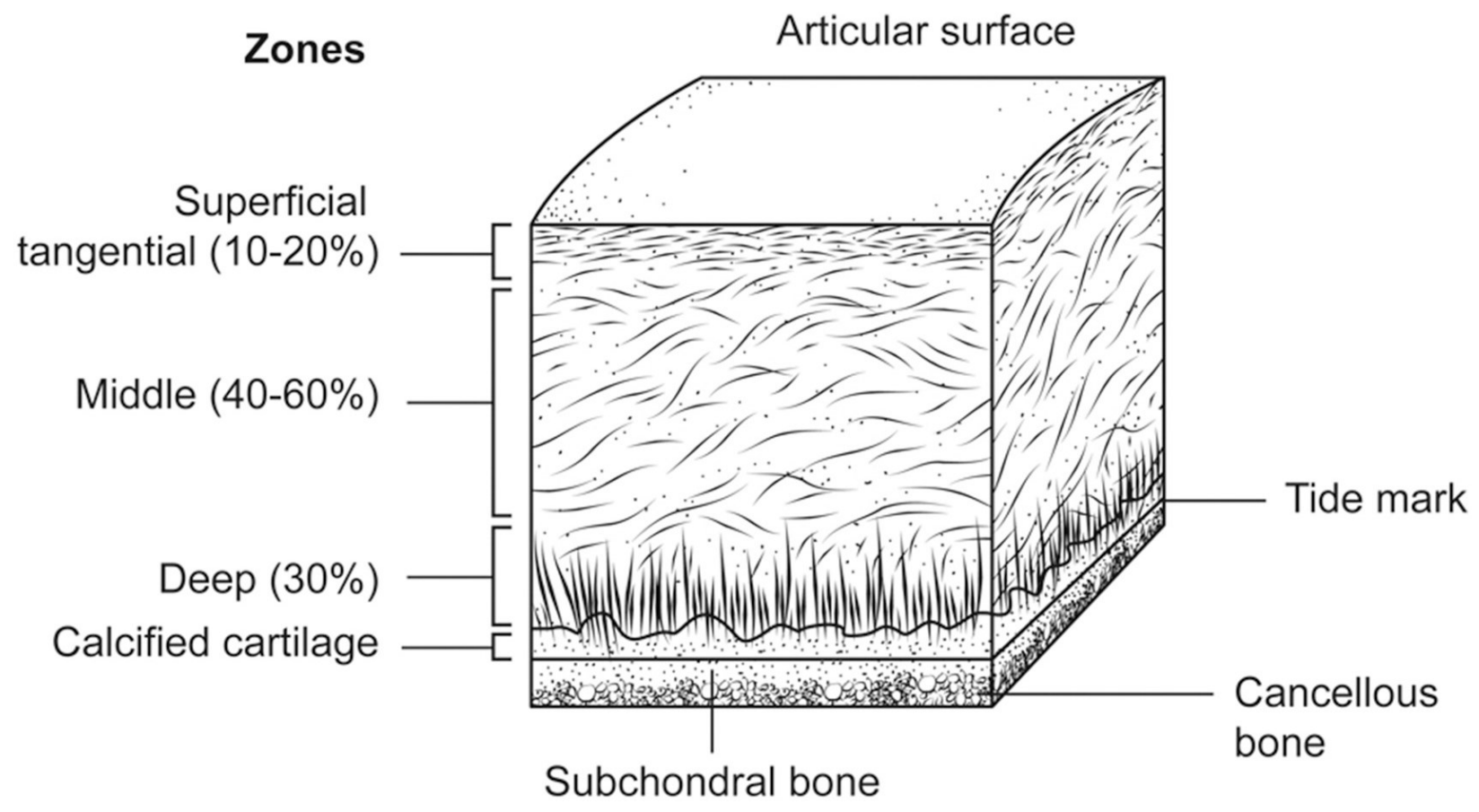

Figure 1.3: Depth dependent structure and composition of articular cartilage. Reprinted from Phys Ther Sport, 16(4), Brody L.T., "Knee osteoarthritis: Clinical connections to articular cartilage structure and function", pg. 302, Copyright (2015), with permission from Elsevier.

\subsection{Osteoarthritis}

In spite of its prevalence the specific causes of OA are not well understood [24]. The onset of the disease is typically classified as either primary or secondary with the latter being an indirect result of trauma, obesity, malposition, or other sources [6]. The degeneration of the cartilage is typically accompanied by a remodeling of the underlying subchondral bone, development of osteophytes, narrowing of the joint space, and various biochemical changes in the joint as a whole [4, 9]. Degradation of the synovial fluid is also typical in osteoarthritic joints, hindering its fluid film lubrication properties [13].

The early stages of the disease are thought to be a result of localized disturbances in the catabolic and anabolic regulation of the ECM by chondrocytes and matrix proteinases [9, 25]. Once these disruptions to regular tissue maintenance pass a threshold the damage becomes irreversible [6]. As this progresses the associated destruction of the ECM is accompanied by a depletion in proteoglycan content, 
particularly near the surface, which further exacerbates the matrix degradation [4, 9]. This alteration in tissue composition results in an increase in water content and tissue permeability. This has been shown to be a side effect of the reduced flow regulating capabilities by the remaining proteoglycan content [26, 27]; dramatically altering the mechanical properties of the tissue [28]. The net result of these changes is reduced interstitial fluid pressurization of the cartilage and a reduction of its compressive stiffness, particularly in the dynamic phases of loading [29]. In late stages of OA, the tensile stiffness of cartilage can be reduced up to $90 \%$ compared to healthy tissue [19].

Once symptoms develop, damage is typically graded using scales associated with physical evaluations and medical imaging [6]. A commonly used scale for OA classification is the Kellgren and Lawrence grade based on radiological assessments of the joint [30]. Diagnosis and monitoring of the disease in early stages of development is difficult due to subtle changes thought to be mainly biochemical in nature. However, use of vibrational spectroscopy in arthroscopic joint surgeries has recently shown potential in this area [31-33]. Near-infrared spectroscopy, with the use of machine learning, has demonstrated reliable predictions of proteoglycan content in the superficial zone of cartilage [31] and related mechanical properties arthroscopically [32]. The use of Raman spectroscopy has also shown similar potential arthroscopically for the differentiation of heathy vs. OA cartilage [33].

\subsection{Raman spectroscopy}

Raman spectroscopy has been shown capable of not only discerning minute changes to the structure of cartilage $[24,34,35]$, but also determining macro-scale composition and organization in tissue crosssections $[20,21]$.

In a simplified manner spontaneous Raman scattering of light can be explained as follows. When a material is irradiated with light, the oscillating electric field of the light sets the electrons of its 
constituent molecules into oscillatory motion. This induces an electric dipole moment, which can be expressed mathematically in the simplified case of a diatomic molecule as in Eq.(1). Here $\mu_{m}(t)$ represents the dipole moment as a function of time $t, \alpha(t)$ represents the polarizability, c. c. is the complex conjugate, and $E_{0}$ and $\omega_{0}$ represent the vibrational amplitude and frequency of an incident electric field $(E(t))$ respectively.

$$
\mu_{m}(t)=\alpha(t) E(t)=\alpha(t)\left(E_{0} e^{-j \omega_{0} t}+\text { c.c. }\right)
$$

However, the electrons are bound to the nuclei. As a result the electron polarizability depends on the nuclear motion $Q(t)$ and can be expressed as a Taylor series expansion as shown in Eq.(2)[36]. In this equation $\alpha_{0}$ represents the polarizability at the equilibrium position [37].

$$
\alpha(t)=\alpha_{0}+\left(\frac{\partial \alpha}{\partial Q}\right)_{0} Q(t)+\cdots
$$

The nuclear motion along $Q$ (shown in Eq. (3))can be assumed to be that of a classical harmonic oscillator [36], and specific vibrational modes have a non-zero rate of change in polarizability with respect to nuclear displacement. In Eq. (3) $Q_{0}$ is the amplitude of the nuclear motion, $\omega_{m}$ represents the nuclear resonance frequency, and $\phi$ is the phase of the nuclear mode vibration [36].

$$
Q(t)=2 Q_{0} \cos \left(\omega_{m} t+\phi\right)=Q_{0}\left[e^{j \omega_{m} t+j \phi}+e^{-j \omega_{m} t-j \phi}\right]
$$

The induced dipole moment radiating light can now be expressed through Eq. (4) [37].

$$
\mu_{m}(t)=\alpha_{0} E_{0} e^{-j \omega_{0} t}+E_{0}\left(\frac{\partial \alpha}{\partial Q}\right)_{0} Q_{0}\left[e^{-j\left(\omega_{0}-\omega_{m}\right) t+j \phi}+e^{-j\left(\omega_{0}+\omega_{m}\right) t-j \phi}\right]+c . c .
$$

The first term $\left(\alpha_{0} E_{0} e^{-j \omega_{0} t}\right)$ represents an oscillating dipole moment radiating light with the incident beam frequency $\omega_{0}$. This corresponds to elastic Rayleigh scattering. The second and third oscillating terms represent radiation of inelastic Raman scattered light with a shifted frequency; $\left(\omega_{0}+\omega_{m}\right)$ for 
anti-Stokes and $\left(\omega_{0}-\omega_{m}\right)$ for Stokes. These inelastic Raman scattering modes are only active if the rate of change of the polarizability with respect to the nuclear displacement $\left(\frac{\partial \alpha}{\partial Q}\right)_{0}$ is non-zero [37]. Raman scattering is inherently weak in comparison to Rayleigh scattering, with approximately 1 in $10^{8}$ of photons from the incident beam undergoing spontaneous Raman scattering [38]. Raman spectroscopy is based on detecting the intensity of this scattered light as a function of shifted frequency to understand molecular structure and composition in materials. The shift in frequency from Raman scattering, associated with the change in vibrational energy level, is typically conveyed through a wavenumber $\left(\mathrm{cm}^{-1}\right)$ value expressed through Eq.(5). In this equation $\lambda_{i}$ represents the wavelength of the incident beam, and $\lambda_{\text {shift }}$ represents the wavelength of the scattered light.

$$
\text { Wavenumber }\left(\mathrm{cm}^{-1}\right)=\frac{1}{\lambda_{i}}-\frac{1}{\lambda_{\text {shift }}}
$$

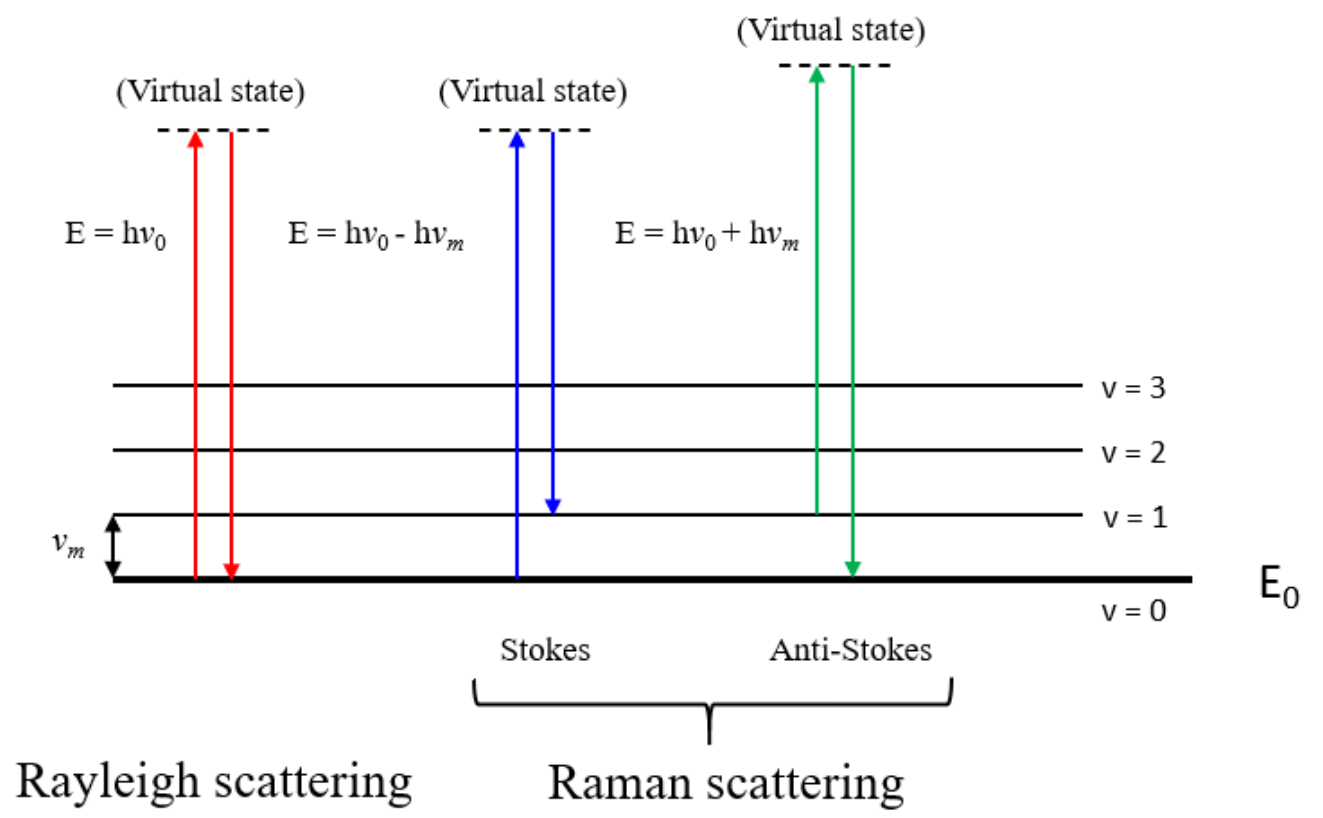

Figure 1.4: Molecular energy level diagram illustrating the difference between Rayleigh and Raman scattering $\left(\mathrm{E}_{0}=\right.$ electronic energy state, $\mathrm{E}=$ scattering photon energy, $\mathrm{v}=$ vibrational energy state, $v_{m}=$ shift in scattering frequency associated with a change in vibrational energy levels, $v_{0}=$ incident electric field vibrational frequency, $\mathrm{h}=$ Planck's constant). 
A simplified visual depiction of how elastic and inelastic scattering differ is shown in Figure 1.4, in

reference to the energy state of a molecule.

A table of tentatively assigned vibrational modes associated with articular cartilage Raman bands are given below based on past studies [16, 21, 34, 39].

Table 1.1: Tentatively assigned Raman bands with molecular vibration modes in articular cartilage based on past studies $[16,21,34,39]$.

\begin{tabular}{|c|c|}
\hline Raman shift $\left(\mathrm{cm}^{-1}\right)$ & Assignment \\
\hline 760 & Tryptophan ring deformation \\
\hline 816 & Collagen/proteins, C-C stretch protein backbone \\
\hline 856 & Collagen, C-C stretch (Proline) \\
\hline 875 & Collagen, C-C stretch (Proline/Hydroxyproline) \\
\hline 920 & Collagen, C-C stretch (Proline/Hydroxyproline) \\
\hline 938 & $\begin{array}{l}\text { Collagen, C-C stretch ( } \alpha \text {-helix, Proline); } \\
\text { GAG, C-O-C stretch (CS) }\end{array}$ \\
\hline 958 & Bone, $\mathrm{PO}_{4}{ }^{3-}$ stretch (phosphated hydroxyapatite) \\
\hline 1003 & C-C symmetric ring stretch (Phenylalanine) \\
\hline 1033 & C-C symmetric ring stretch (Phenylalanine) \\
\hline 1042 & Pyranose ring stretch \\
\hline $1063-1068$ & GAG, $\mathrm{SO}_{3}{ }^{-}$stretch (CS) \\
\hline 1126 & Proteins, $\mathrm{C}-\mathrm{C} / \mathrm{C}-\mathrm{OH} / \mathrm{C}-\mathrm{N}$ stretching; $\mathrm{C}-\mathrm{O}-\mathrm{C}$ stretch glycosidic linkages \\
\hline 1163 & Pyranose ring stretch; $\mathrm{C}-\mathrm{H}$ bending (Tyrosine) \\
\hline 1207 & $\mathrm{CH}_{2}$ twist (Hyaluronic acid) \\
\hline 1235 & Collagen, random coil (Amide III) \\
\hline 1245 & Collagen, $\mathrm{NH}_{2}$ bending (random coil, Amide III) \\
\hline 1269 & Collagen, $\mathrm{NH}_{2}$ bending ( $\alpha$-helix, Amide III) \\
\hline 1313 & $\mathrm{CH}_{2} / \mathrm{CH}_{3}$ twisting \\
\hline 1343 & GAG, $\mathrm{CH}_{2} / \mathrm{CH}_{3}$ wagging \\
\hline 1370 & COO symmetric stretch (Hyaluronic acid) \\
\hline 1380 & GAG, $\mathrm{CH}_{3}$ deformation \\
\hline 1424 & GAG, COO symmetric stretch \\
\hline 1450 & Collagen/proteins $\mathrm{C}-\mathrm{H}$ bending $\left(\mathrm{CH}_{2} / \mathrm{CH}_{3}\right)$ \\
\hline 1557 & Amide II/C=C aromatic ring stretch (Phenylalanine, Tryptophan) \\
\hline 1606 & $\mathrm{C}=\mathrm{C}$ aromatic ring stretch (Phenylalanine, Tryptophan) \\
\hline 1640 & Amide I/Collagen secondary structure \\
\hline 1655 & Amide I ( $\alpha$-helix) \\
\hline 1668 & Collagen, random coil (Amide I) \\
\hline 1685 & Amide I ( $\beta$ - sheet) \\
\hline
\end{tabular}


Several studies have taken advantage of ratios in vibrational mode intensity peaks, or integrated areas, to interpret structural changes in connective tissues and cartilage. One of these is the $938 / 922 \mathrm{~cm}^{-1}$ ratio to assess hydrogen bond formation between proline-hydroxyproline linkages and water molecules, related to collagen stability [40-42]. In another study Takahashi et al.[35] were able to draw a correlation between OA severity and disorder in the collagen secondary protein structure. This was done using the $1246 / 1269 \mathrm{~cm}^{-1}$ Amide III doublet ratio to quantify the abundance of random coil to $\alpha$-helix structures. Kumar et al.[24] also used this doublet ratio in a similar study to show that significant changes in magnitude were a potential marker for early OA development. However, Bonifacio et al.[43] were also able to demonstrate this doublet ratio is dependent on sample orientation, potentially influencing these findings. The $856 / 875 \mathrm{~cm}^{-1}$ ratio has also been used to assess collagen stability in mechanically stressed cartilage [34]. This ratio was used based on evidence of chondrocytes converting proline to hydroxyproline during cartilage synthesis in disrupted tissue.

While variations in the intensity of individual Raman bands are informative, many macro-molecules have multiple vibrational modes which can also overlap with others in a mixture. Because of this, multivariate analysis is a useful tool for quantifying signal composition in Raman spectroscopy [44]. One form of this analysis is multivariate curve resolution (MCR) [45].

\subsection{Raman based-multivariate curve resolution}

The aim of MCR is to determine the contributions of sub-components in a mixture to the overall sample signal [46]. This is possible with Raman spectroscopy due to the property that Raman scattering intensity is proportional to the density of scattering molecules $[37,47]$. Sub-component reference spectra, of known dilution, can then be used in a correlation curve to find the concentration of analyte in the Raman spectrum of a mixture. These individual spectral contributions can be expressed 
mathematically through Eq.(6). In this equation $D$ represents the combined mixture spectra, and C represents a single matrix of relative concentrations for the individual pure reference spectra represented by the matrix S. Finally, $M_{\text {Error }}$ represents the remaining error in the fit $[46,48]$.

$$
D=\sum C S+M_{\text {Error }}
$$

A MATLAB (MathWorks, Mass., U.S.A.) toolbox called MCR-ALS developed by Jaumot et al. [48] is specifically made for this deconvolution of spectra. The software uses an alternating least squares (ALS) algorithm to minimize error iteratively when curve fitting pure reference spectra and concentration matrices to the mixed signal. This error can be quantified by an $R^{2}$, or a percent lack of fit (LoF(\%)) value. The expressions for these terms are given by Eq.(7) and (8) respectively; where $d_{i j}$ is an element of the experimental data matrix, and $e_{i j}$ is the residual obtained from taking the difference between corresponding experimental and predicted values $[46,48]$.

$$
\begin{gathered}
R^{2}=100 \cdot \sqrt{\frac{\sum_{i, j} d_{i j}^{2}-\sum_{i, j} e_{i j}^{2}}{\sum_{i, j} d_{i j}^{2}}} \\
\operatorname{LoF}(\%)=100 \cdot \sqrt{\frac{\sum_{i, j} e_{i j}^{2}}{\sum_{i, j} d_{i j}^{2}}}
\end{gathered}
$$

A recent study by Bergholt et al.[20] applied Raman spectroscopy-based MCR to investigate crosssections of native and engineered cartilage for relative spectral contributions of collagen II, GAG, and water. This study also used anisotropic scattering properties to obtain information about collagen fibril orientation. The same group also showed that the absolute concentration was linearly proportional to MCR sub-component results [21].

These studies show the feasibility of Raman spectroscopy-based determination of composition using MCR analysis applied to tissue cross-sections. However, cross-sectioning requires the destruction of 
tissues and is not practical in-situ or in-vivo. Measurements through-the-surface keeps the morphology intact, but the optical signal attenuates significantly with depth as a consequence of the heterogenous composition of cartilage $[49,50]$.

\subsection{Optical clearing}

In a simplified description, transparency in materials such as glass or air is partly related to their homogeneous composition [49]. When incident light strikes these materials the molecules affected together in a plane will scatter light spherically in all directions, out of phase from the primary wave front $[49,51]$. The majority of scattered light not traveling along this primary wave front direction will destructively interfere with the scattered light from surrounding molecules. This is due to the proximity of scattering molecules in the material, resulting in most scattered wavelets meeting a destructively interfering counterpart. However, scattered light will constructively interfere in the forward direction of the primary wave front as a result of all scattering wavelets oscillating in the same phase $[49,51]$. This constructive interference allows the wave to propagate through the material with minimal scattering in other directions. In the case of biological tissues, the medium is typically a heterogeneous mixture of structures with varying composition and refractive indices. This differing composition means that scattered light from molecules in the same plane do not always have a destructively interfering counterpart. Numerous scattering molecules are then able to propagate light in all directions within the tissue, resulting in a turbid appearance [49].

Optical clearing of tissue to reduce this scattering heterogeneity is typically approached in three main ways [52]. The simplest mechanism involves passive immersion in an optical clearing agent (OCA). This replaces the interstitial fluid with a liquid that more closely matches the refractive index of the remaining ECM. The other two mechanisms involve the removal of lipids followed by refractive index 
matching through either hydration, dehydration, or the previously-mentioned immersion method [49, $50,52]$. These clearing mechanisms often lead to alterations in tissue morphology. However, studies involving passive immersion of cartilage in solutions have shown that a significant improvement in optical properties is possible with minimal damage to the ECM. Neu et al.[53] demonstrated this for cartilage up to a depth of $2.5 \mathrm{~mm}$ with a fructose-based OCA similar to SeeDB [54]. Another study by Bykov et al.[55] showed that optical clearing of cartilage with iohexol could enable visibility of the cartilage-subchondral bone interface with optical coherence tomography in as little as 15 minutes. This optically cleared cartilage offers improved scattering properties for spectroscopic measurements made through-the-surface.

\subsection{Cartilage Modeling with Finite Element Analysis}

A practical application of compositional information obtained from Raman spectroscopy is in finite element (FE) modeling of cartilage. Use of imaging techniques for applying the structural hierarchy in cartilage to FE models $[56,57]$ has proven invaluable in understanding the mechanical contributions of each sub-component. In particular the enhanced interstitial fluid pressurization, and associated fluid load support, generated in these depth-dependent models has been routinely reported due to its importance in reducing surface friction and wear in cartilage [56, 58-60]. To date, use of Raman spectroscopy for applying this structural hierarchy to FE models of cartilage has not been reported.

Accurate FE modeling of articular cartilage is challenging due to the complex structure and organization of the tissue described above. The tissue exhibits heterogeneity and anisotropy when loaded in tension and compression [61-64]. In addition the tissue is viscoelastic, exhibiting a time-dependent mechanical response $[15,65-67]$. Traditionally, the tissue has been computationally modeled as a biphasic material $[15,66]$. To capture the tension-compression nonlinearity in FE modeling more recent studies have 
simulated the tissue as a biphasic poroviscoelastic (BPVE) material with fibril reinforcement $[56-58,62$, 63, 68-70]. Constitutive models incorporating conewise linear elasticity have also been shown to reproduce accurate tissue response to loads $[61,62]$.

Adjusting the tissue response of these FE models to match experimentally recorded results offers a means of quantifying sample-specific material properties in cartilage. Three loading configurations commonly used for this purpose are confined compression, unconfined compression, and indentation (illustrated in Figure 1.5) $[63,71,72]$. These are typically used to load the tissue dynamically, or in stressrelaxation or creep. Indentation testing is of particular interest for this thesis as it offers the most convenient means of testing cartilage in-situ or in-vivo with minimal sample preparation and is less sensitive to the natural surface curvature. However, it is characterised by complex boundary conditions, and, with the exception of linear elastic materials, requires FE models to assess material parameters.

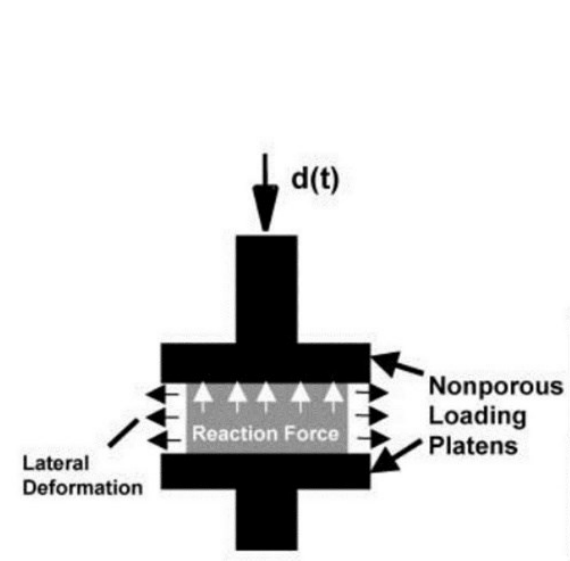

Unconfined compression

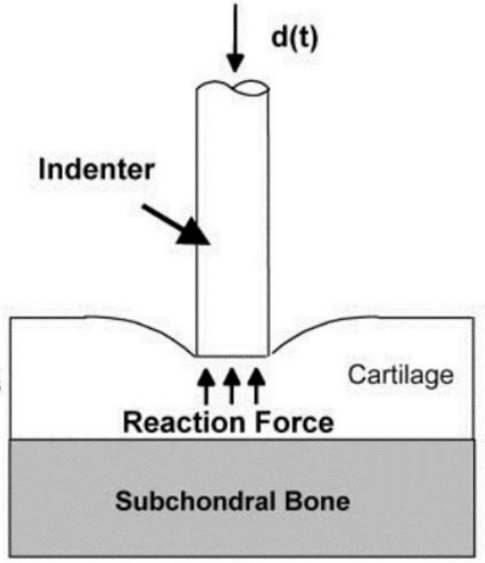

Indentation

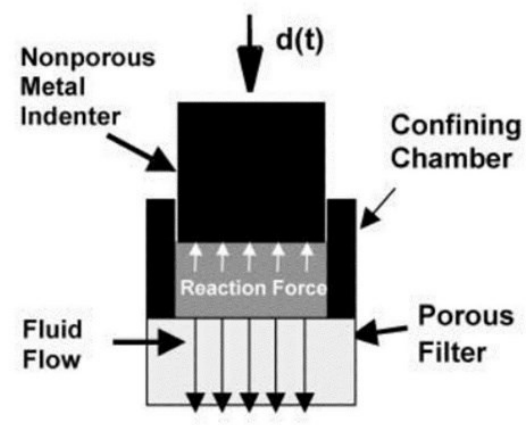

Confined compression

Figure 1.5: Typical loading configurations used for testing of cartilage. Adapted from J. Biomech, 34(4), M. DiSilvestro and J. Suh, "A cross-validation of the biphasic poroviscoelastic model of articular cartilage in unconfined compression, indentation, and confined compression", pg. 521, Copyright (2001), with permission from Elsevier. 
A specific fibril reinforced BPVE model for cartilage which has been shown to predict tissue response well is the ellipsoidal continuous fiber distribution (CFD) model proposed by Ateshian et al.[63]. This model has been shown to be particularly accurate in reproducing tissue response in the flowindependent stage of loading.

\subsection{Finite Element Constitutive Theory}

A commonly used software for non-linear finite element analysis of biological tissues is FEBio. This is an open source program which was developed between Columbia university and the university of Utah [73] specifically for the analysis of biological tissues. It implements the implicit finite element method with common tissue material models. To account for the solid structure of the articular cartilage the ellipsoidal CFD can be implemented within FEBio 2.9 in conjunction with a supporting ground substance. The Cauchy stress and spatial elasticity tensor in the fiber portion of the solid matrix can be described by Eq.(9) and (10), respectively $[63,73]$. In these equations $H$ represents a unit step function imposing contributions only in tension, $I_{n}$ represents the square of the fiber stretch, and $n^{r}$ represents a unit vector in the reference configuration which a fiber is oriented along (based on global orthonormal spherical coordinates $(\varphi, \theta))$. Finally, $\overline{\bar{t}}_{n}\left(n^{r}\right)$ and $\overline{\bar{C}}_{n}\left(n^{r}\right)$ represent the Cauchy stress and spatial elasticity tensors for fibers initially oriented along $n^{r}$ respectively [63].

$$
\begin{aligned}
& \overline{\bar{\sigma}}_{f}=\int_{0}^{2 \pi} \int_{0}^{\pi} H\left(I_{n}-1\right) \overline{\bar{t}}_{n}\left(n^{r}\right) \sin (\varphi) d \varphi d \theta \\
& \overline{\bar{C}}_{f}=\int_{0}^{2 \pi} \int_{0}^{\pi} H\left(I_{n}-1\right) \overline{\bar{C}}_{n}\left(n^{r}\right) \sin (\varphi) d \varphi d \theta
\end{aligned}
$$


These equations can be connected to the fiber strain energy density function through standard finite elasticity relations expressed by Eq.(11) and (12) [63]. In these equations J represents the determinant of the deformation gradient tensor $\overline{\bar{F}}$, and $N=n \otimes n=I_{n}{ }^{-1} \cdot \overline{\bar{F}} \cdot N^{r} \cdot \overline{\bar{F}}^{T}$ with $n=I_{n}{ }^{-1 / 2} \overline{\bar{F}} \cdot n^{r}$ representing the fiber direction in the deformed state [63]. In these equations $\otimes$ represents the tensor product.

$$
\begin{gathered}
\overline{\bar{t}}_{n}=2 J^{-1} I_{n} \cdot \frac{\partial \Psi}{\partial I_{n}} \cdot N \\
\overline{\bar{C}}_{n}=4 J^{-1} I_{n}{ }^{2} \frac{\partial^{2} \Psi}{\partial I_{n}{ }^{2}} N \otimes N
\end{gathered}
$$

The strain energy density function ( $\Psi$ ) for the fibers is given by Eq.(13) where $\beta \geq 2$ and $I_{n} \geq 1$. In this equation $\xi\left(n^{r}\right)$ and $\beta\left(n^{r}\right)$ are variable material properties along the direction $n^{r} \quad$ [63]. The former parameter is related to the fiber tensile modulus in units of force per unit area, the latter parameter is unitless.

$$
\Psi\left(n^{r} ; I_{n}\right)=\xi\left(n^{r}\right)\left(I_{n}-1\right)^{\beta\left(n^{r}\right)}
$$

$\xi\left(n^{r}\right)$ and $\beta\left(n^{r}\right)$ can be expressed in the local orthonormal coordinates $(\Theta, \Phi)$ as seen in Eq.(14) and $(15)[63,73] . \xi_{1-3}$ and $\beta_{1-3}$ represent the corresponding material parameters along the respective semiaxes of the ellipsoid in the local coordinate system [63].

$$
\begin{aligned}
& \xi\left(n^{r}\right)=\left(\frac{\cos ^{2} \Theta \sin ^{2} \Phi}{\xi_{1}^{2}}+\frac{\sin ^{2} \Theta \sin ^{2} \Phi}{\xi_{2}^{2}}+\frac{\cos ^{2} \Phi}{\xi_{3}^{2}}\right)^{-\frac{1}{2}} \\
& \beta\left(n^{r}\right)=\left(\frac{\cos ^{2} \Theta \sin ^{2} \Phi}{\beta_{1}^{2}}+\frac{\sin ^{2} \Theta \sin ^{2} \Phi}{\beta_{2}^{2}}+\frac{\cos ^{2} \Phi}{\beta_{3}^{2}}\right)^{-\frac{1}{2}}
\end{aligned}
$$


When $\beta=2$ this results in a jump at the strain origin for the elasticity modulus $\overline{\bar{C}}_{n}$, creating a piecewise-linear stress strain relationship for fibers transitioning to tension at small deformations [63]. The ground substance supporting the CFD, aimed at accounting for the proteoglycans, is modeled as a compressible neo-Hookean hyperelastic material. The strain energy density function governing this material is given by Eq.(16) [73]. In this equation $\mu$ and $\lambda$ represent Lamé parameters, and $I_{1}$ represents the first invariant of the right Cauchy-Green deformation tensor [73].

$$
\Psi=\frac{\mu}{2}\left(I_{1}-3\right)-\mu \ln (J)+\frac{\lambda}{2}(\ln J)^{2}
$$

The Cauchy stress and elasticity tensors in the ground substance can be described by Eq.(17) and (18) respectively [73]. In these equations $B$ represents the left Cauchy-Green deformation tensor, and $I$ represents the identity tensor. The $\overline{\bar{\otimes}}$ operator represents a variation of the tensor product, detailed by Eq. 2.1.24 in the FEBio theory manual.

$$
\begin{gathered}
\overline{\bar{\sigma}}_{m}=\frac{\mu}{J}(B-I)+\frac{\lambda}{J}(\ln J) I \\
\overline{\bar{C}}_{m}=\frac{\lambda}{J}(I \otimes I)+\frac{2}{J}(\mu-\lambda \ln (J)) I \underline{\otimes} I
\end{gathered}
$$

To adequately describe biphasic porous media an expression is needed to relate the momentum transfer between the solid phase deformation and the fluid phase pressure. This is done within FEBio using the linear momentum equation for the fluid phase, and adding a term $\left(\hat{P}_{d}^{s}\right)$ representing this momentum exchange through frictional forces [73]. Combined with the constitutive relationship of $\hat{P}_{d}^{s}=-\varphi^{s} k^{-1} \cdot w$ the momentum equation can be rearranged to express the fluid flux, $w$, as in Eq.(19) which is equivalent to Darcy's law [73]. Within these expressions $\varphi^{s}$ represents the solid phase porosity, $\nabla p$ represents the gradient of the fluid pressure, $k$ represents the symmetric hydraulic 
permeability tensor, $\rho^{f}$ represents the true fluid density, and $b^{f}$ represents the body force effects per units mass acting on the fluid phase [73].

$$
w=-k \cdot\left(\nabla p+\rho^{f} b^{f}\right)
$$

It is important to note that the viscous shear forces are neglected within this framework [73], and it is only appropriate for low Reynolds number flows. This is deemed a valid approximation due to the low permeability of the tissue, on the order of $1 \times 10^{-15} \mathrm{~m}^{4} / \mathrm{N} \cdot \mathrm{s}[15]$.

The total stress within the tissue can now be described as the sum of the fiber stress, ground substance stress, and the fluid pressure shown by Eq.(20).

$$
\sigma_{t}=\left(\sigma_{f}+\sigma_{m}\right)-p I
$$

\subsection{Material parameter optimization}

Material parameter estimations for the constitutive model described above can be carried out with the built-in FEBio optimization program. This optimization is based on the Levenberg-Marquardt non-linear least squares algorithm [73]. The goal of this algorithm is to minimize an expression for the difference, $f(a)$, between experimental data points $\left(y_{i}, x_{i}\right)$ and model data points $y\left(x_{i} ; a\right)$ created from iteratively varied input parameters. In this case the input parameters are material parameters for the ground substance Young's Modulus, the fiber tensile modulus parameter, and the hydraulic permeability. The experimental data points given to the algorithm are typically the reaction forces measured by a load cell.

$$
f(a)=\sum_{i=1}^{n}\left[y_{i}-y\left(x_{i} ; a\right)\right]^{2}
$$




\subsection{Thesis objectives}

This thesis has two objectives. First, to assess the feasibility of using Raman spectroscopy-based MCR for determining cartilage sub-component distributions through-the-surface. Further to this, assess if OCAs can improve the depth of resolution. Second, to apply this composition information to FE models of cartilage to assess accuracy in predicted tissue response.

The net result of achieving these objectives will assess the viability and application of Raman-based MCR for determining cartilage sub-component distributions in-situ or in-vivo. It will also enable FE modeling of cartilage based on composition. A more detailed understanding of depth-dependent mechanical response to loading, i.e. based on depth-dependent composition, is also required to study the mechanoregulation of chondrocyte metabolism in maintaining tissue health and in processes such as OA. This sample specific means of data acquisition may then facilitate better understanding of how observed compositional changes in diseased tissue affect predicted material responses. This is particularly advantageous in monitoring of early stages or mild OA, which can be characterized by a severe depletion of proteoglycan content $[4,9]$. As has been previously demonstrated, this measurement method would be applicable for assessment in-vivo [33].

\subsection{Thesis outline}

The thesis objectives will be assessed in the following chapters based on the work from two manuscripts as follows:

1) In the first manuscript, the feasibility of using Raman spectroscopy-based MCR composition information through-the-surface was evaluated. To do this Raman-based MCR results throughthe-surface, before and after optical clearing, are compared to those from cross-sections used as standards. Absolute sub-component concentrations are then estimated using cross-sectioned 
MCR results in conjunction with sample volumes and weights. The effects of a fructose-based OCA similar to FRUIT [74] are also evaluated to see if it can improve the depth of resolution for distribution measurements through-the-surface.

2) In the second manuscript, composition information obtained from cross-sections, and throughthe-surface, was applied to FE models of cartilage undergoing stress-relaxation indentation. These depth-dependent composition models were evaluated against homogeneous distribution models to compare accuracy in predicted tissue response. These comparisons will also determine to what extent models using Raman data obtained through-the-surface match their equivalent cross-section counterparts. 


\section{Chapter 2:}

\section{Measurement of cartilage sub-component distributions through the surface by Raman spectroscopy-based multivariate analysis}

This chapter was published in the Journal of Biophotonics as:

Mason, D.R., Murugkar, S. and Speirs, A.D. (2020), Measurement of cartilage sub-component

distributions through the surface by Raman spectroscopy-based multivariate analysis. J. Biophotonics.

Accepted Author Manuscript. doi:10.1002/ibio.202000289

The chapter presented reflects a version of this published manuscript, adapter for formatting within the thesis and based on examiner recommendations. 


\section{Measurement of cartilage sub-component distributions through the surface by Raman spectroscopy-based multivariate analysis}

\subsection{Abstract}

Articular cartilage possesses unique material properties due to a complex depth-dependent composition of sub-components. Raman spectroscopy has proven valuable in quantifying this composition in cartilage cross-sections. However, cross-sectioning requires tissue destruction and is not practical in-situ or in-vivo. In this work, Raman spectroscopy-based multivariate curve resolution (MCR) was employed in porcine cartilage samples $(n=12)$ to measure collagen, glycosaminoglycan (GAG), and water distributions through-the-surface for the first time; these were compared against cross-section standards. Through-the-surface Raman measurements proved reliable in predicting composition distribution up to a depth of approximately $0.5 \mathrm{~mm}$. A fructose-based optical clearing agent (OCA) was also used in an attempt to further improve depth-of-resolution of this measurement method. However, it did not, mainly due to a high spectral overlap with the Raman spectra of main cartilage subcomponents. This measurement technique potentially could be used in-situ or in-vivo, to better understand the etiology of joint diseases such as osteoarthritis (OA).

\subsection{Introduction}

Articular cartilage serves as the bearing surface for bone-to-bone load transmission, and enables nearly friction-free joint movement [2]. Fulfilling this role requires unique material properties that are derived from its complex composition and organization. Past studies using Raman spectroscopy have reported the relative distribution of this composition in-vitro with cross-sectioning [20,21], and related it to concentrations determined from assay tests. However, quantifying the composition with depth throughthe-surface will keep morphology intact, and enable in-situ or in-vivo measurements. This could offer 
better insight into how joint diseases such as OA develop, especially in the early stages, and how composition affects functionality.

Cartilage consists of a dense extracellular matrix (ECM) predominantly made up of collagens, proteoglycans, and water as well as other proteins and molecules in lower concentrations [2, 39].

Recently, tools such as Raman spectroscopy have proven capable of not only quantifying these subcomponent concentrations, but also observing subtle variations in their structural organization [34, 35].

While variations in the intensity of individual Raman bands are informative, many molecules have multiple vibrational modes which can also overlap with others in a mixture. Because of this, multivariate analysis is a useful tool for quantifying signal composition in Raman spectroscopy [44]. One form of this analysis is multivariate curve resolution (MCR). MCR takes advantage of the property that the intensity of Raman scattered light is proportional to the density of scattering molecules $[37,47]$, and uses this to decompose a mixed spectrum into relative concentrations of pure sub-component spectra. A recent study by Bergholt et al. [20] used MCR to investigate cross-sections of native and engineered cartilage for determining relative spectral contributions of collagen II, glycosaminoglycan (GAG), and water. This study also used anisotropic scattering properties to obtain information about collagen fibril orientation. The same group also showed that the absolute concentration of sub-components was linearly proportional to MCR results [21].

These studies show the feasibility of Raman spectroscopy-based determination of composition using MCR analysis applied to tissue cross-sections. However, cross-sectioning requires the destruction of tissues and is not practical in-situ or in-vivo. Measurements made through-the-surface keep morphology intact, but the optical signal attenuates significantly with depth as a consequence of the heterogenous composition of cartilage $[49,50]$. The use of an optical-clearing-agent (OCA) can overcome some of this optical attenuation in turbid tissues [49]. OCAs vary in clearing technique, but commonly involve passive 
immersion in a solution replacing the interstitial fluid with a liquid that more closely matches the refractive index of the remaining ECM [49]. Previous studies focusing on the effects of clearing on cartilage have shown that a significant improvement in optical properties is possible with minimal damage to the ECM. A study by Neu et al.[53] illustrated this using a fructose-based OCA to clear cartilage up to a depth of $2.5 \mathrm{~mm}$.

The aim of this study is to assess the feasibility of Raman spectroscopy and MCR for determining cartilage composition in a novel manner through-the-surface, and whether it could be improved using a fast-acting fructose-based clearing solution similar to FRUIT [74]. FRUIT has been successfully used in the past for clearing mammalian brain tissue [74], and is applied here in articular cartilage for the first time. Non-destructive Raman-based MCR results through-the-surface, before and after clearing, are compared to those from cross-sections used as standards. Absolute sub-component concentrations are then estimated using these cross-sectioned MCR results in conjunction with sample volumes and weights.

\subsection{Materials and methods}

\subsubsection{Specimen preparation}

Skeletally mature porcine knee joints were locally sourced from an abattoir up to 48 hours after slaughter. Cylindrical cartilage plugs $(\varnothing 10 \mathrm{~mm} \times 6 \mathrm{~mm})$ from the trochlear groove or patella were then excised $(n=12)$ from three joints and frozen in a 1xPBS solution. Each sample consisted of up to $2.5 \mathrm{~mm}$ of articular cartilage with the remaining height consisting of the subchondral bone below. Plugs frozen together and used in testing groups were mixed from the three joints to provide more variability. 


\subsubsection{Optical clearing stages}

The timeline of optical clearing stages is illustrated in Figure 2.1. A preclear stage involved samples $(n=$ 6) being thawed for one hour then placed in a stock 1xPBS solution supplemented with an EDTA-free protease inhibitor (SIGMAFAST ${ }^{\mathrm{TM}}$; Sigma-Aldrich Corp., Mo., U.S.A.). Samples then had Raman measurements made through the tissue surface. After being placed in a clearing solution overnight (12 h) at $4{ }^{\circ} \mathrm{C}$, samples were re-measured through-the-surface. This measurement stage was termed postclear. The clearing solution, similar to FRUIT [74], consisted of $40 \% \mathrm{wt} / \mathrm{vol}$ D-(-)-fructose and $24 \%$ wt/vol urea dissolved in distilled water. FRUIT was chosen as the OCA after preliminary tests with fructose required long clearing durations resulting in significant tissue degradation. The clearing window was reduced to $12 \mathrm{~h}$. to minimize this tissue degradation as samples were not fixed. The $40 \%$ FRUIT concentration was chosen to increase diffusion speed into the tissue, due to its lower viscosity, and ensure no precipitation of solutes at the low storage temperature.

Samples were then placed in the stock PBS solution overnight $(12 \mathrm{~h})$ to remove the OCA, and remeasured through-the-surface. This measurement stage was termed recovered. After a needle puncture test to obtain the true thickness, samples were cross-sectioned and spectral measurements repeated. A portion of the cross-sectioned tissue, with bone removed, was weighed wet and placed in an incubator at $37^{\circ} \mathrm{C}$ for 24 hours to dry. It was subsequently weighed and the total water content determined from the weight difference [75].

Control samples $(n=6)$ underwent a similar testing procedure but were not treated with any clearing solution. Raman measurements of the control samples were meant to quantify time-related changes to the cartilage. Only two samples had Raman measurements taken after an elapsed time equivalent to the postclear stage of treated specimens. No significant changes in the Raman spectra of samples was observed between the preclear and recovered stages for both treated and control groups. 
Total testing time lasted four days. Each sample remained at room temperature during testing for approximately 3-4 hours each day, with the remaining time spent at $4{ }^{\circ} \mathrm{C}$.

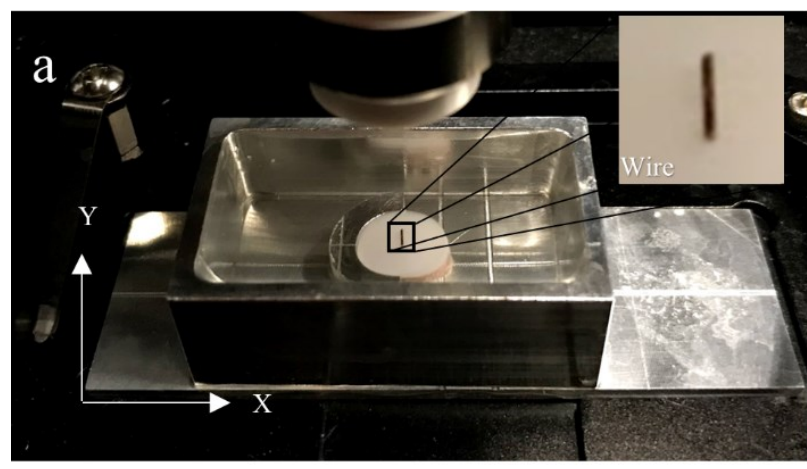

C

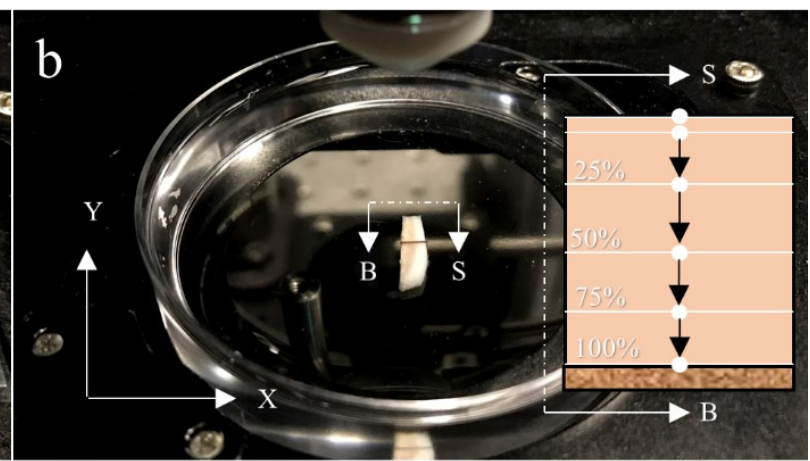

Day 2: Postclear
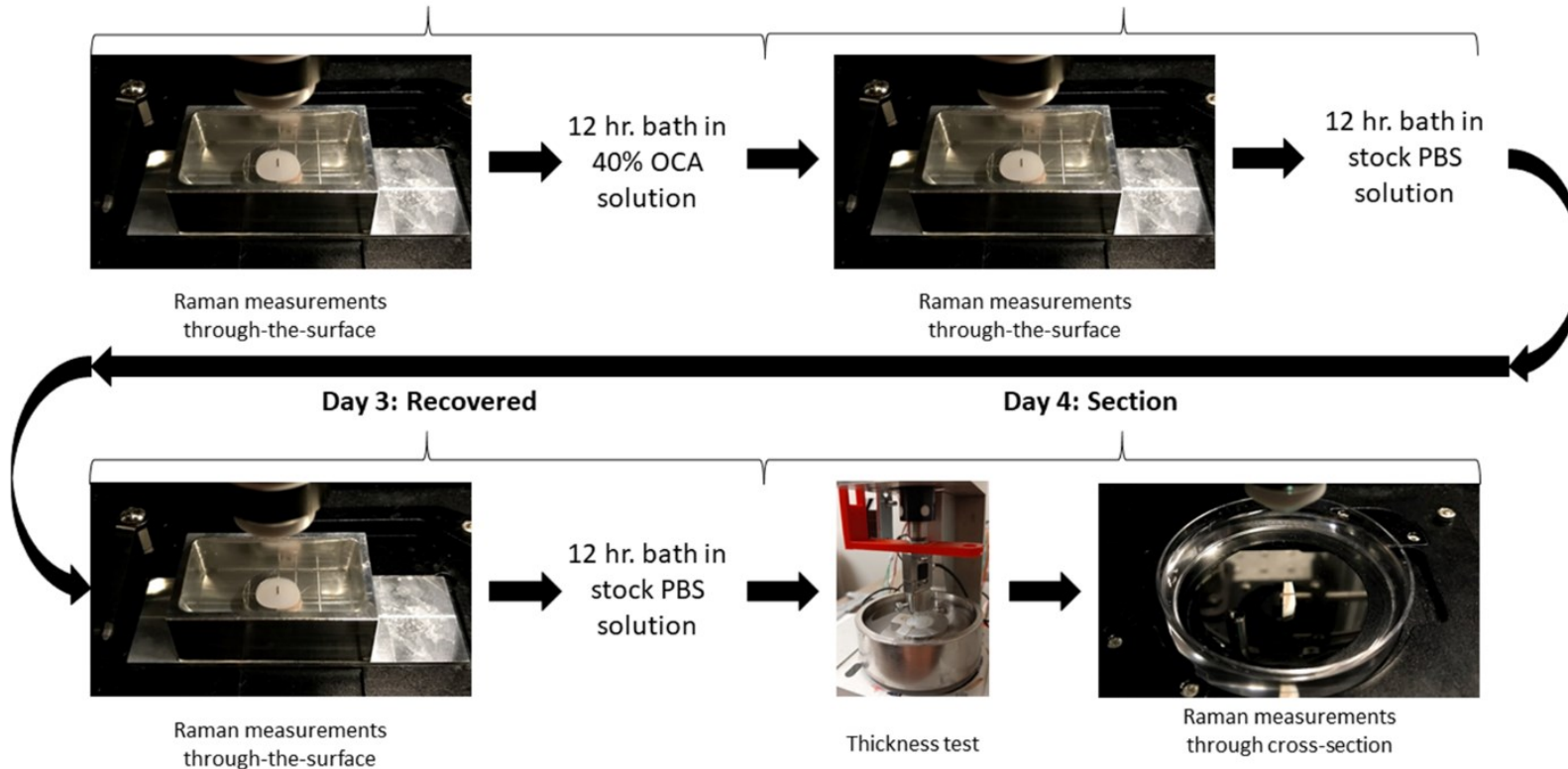

Figure 2.1: (a) Specimen setup for Raman measurements through-the-surface of cartilage. A wire was placed on the bearing surface parallel to the diameter (within $200 \mu \mathrm{m}$ of measurement location) to determine the reference height. (b) Raman measurement of cross-sectioned cartilage plug alongside plug schematic designating spectral measurement locations with $S$ and $B$ indicating the bearing surface and bone respectively. A wire was placed perpendicular to the bearing surface to determine the reference height. (c) Timeline of optical clearing stages. Images (a) and (b) are repeated in (c). 


\subsubsection{Thickness measurement}

To accurately obtain the sample thickness a needle was pushed through the cartilage surface while the reaction force was recorded using a custom-built device [69]. The tissue surface was identified once the reaction force exceeded $5 \mathrm{mN}$. The bone surface was identified by a sudden large change in the reaction force slope with respect to distance. The average sample thickness was $1.9 \pm 0.5 \mathrm{~mm}$. Results are reported as mean $\pm 95 \%$ C.I. unless otherwise stated. Confidence intervals for percentage values $($ LoF(\%)) represent the range of the mean percentage value, not a percentage of the specified value.

\subsubsection{Raman instrumentation}

A lab-built Raman micro-spectroscopy setup [76] was used in this study as shown in Figure 2.2. It consisted of a multimode $785 \mathrm{~nm}$ diode laser (Ondax, CA, U.S.A.) with a maximum output power of 500 $\mathrm{mW}$, that was coupled into a $100 \mu \mathrm{m}$ core, $0.22 \mathrm{NA}$ multimode fiber. Collimated and filtered light from the fiber passed through a 40X, 0.8 NA water immersion microscope objective (Olympus, ON, Canada) to create a laser spot with a lateral diameter and depth of focus of $15 \mu \mathrm{m}$ and $32 \mu \mathrm{m}$ respectively. Raman scattered light from the sample was collected by the same objective and passed through a long-pass dichroic mirror and two long-pass edge filters (Iridian, ON, Canada) to eliminate the $785 \mathrm{~nm}$ laser light. A $300 \mu \mathrm{m}$ core multimode fiber (Thorlabs, NJ, U.S.A.) delivered the Raman signal to a compact spectrometer (Tornado Spectral Systems, ON, Canada). The position of the cartilage sample inside the laser focus (200 mW at the sample) was adjusted by means of an automated stage (FTP 2000, ASI Inc., U.S.A.). Spectra were measured in the $170-2400 \mathrm{~cm}^{-1}$ range, with a spectral resolution of $4 \mathrm{~cm}^{-1}$. For measurement of MCR reference spectra, a 20X, 0.75 NA air objective (Olympus, ON, Canada) was utilized for the dry laboratory grade samples. 


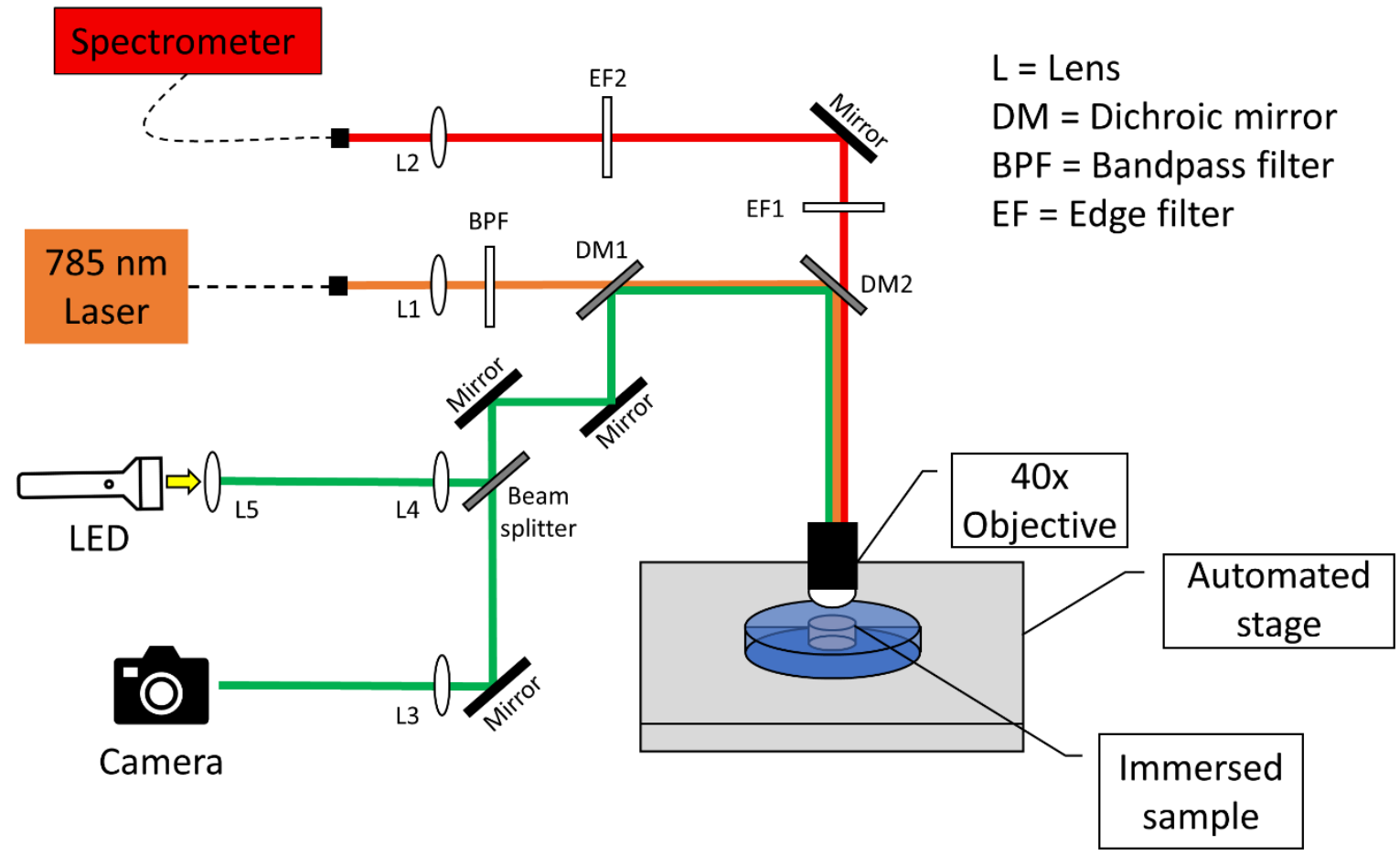

Figure 2.2: Raman spectrometer schematic for measurement of immersed cartilage samples. The specifications of filters, and the CCD camera used, are further detailed in a report by Hansson et al.[76].

\subsubsection{Raman spectroscopy of tissue}

To accurately acquire the $\mathrm{X}-\mathrm{Y}$ position of the cartilage plug center axis, a custom-made sample holder was created as seen in Figure 2.1(a) with known reference dimensions. To determine the surface reference height of the sample the objective was brought to focus on top of a copper wire of known diameter $(254 \pm 4 \mu \mathrm{m})$ placed on the sample surface near the measurement location. Raman spectra were recorded at depths of $0 \mu \mathrm{m}$ and $100 \mu \mathrm{m}$ into the tissue, along with measurements taken at $25 \%$, $50 \%, 75 \%$, and $100 \%$ of the estimated sample thickness.

For cross-section measurements the wire was placed adjacent (+Y direction) to the measurement location, and perpendicular to the bearing surface as seen in Figure 2.1(b), to obtain the reference height and ensure no interfering signal. Raman measurements were taken $100 \mu \mathrm{m}$ into the tissue and found not to deviate parallel to the bearing surface (Y-direction). The cartilage bearing surface was 
deemed to begin where the laser spot could first focus on tissue. Raman spectra were recorded at the same depths stated above, with each measurement being in reference to the top of the wire at new positions.

Raman spectra measured through cross-sections had an acquisition time of $10 \mathrm{~s}$. Through-the-surface measurements had a larger acquisition time of $30 \mathrm{~s}$ to improve the signal-to-noise ratio at depth. Each spectrum was averaged over 15 acquisitions. No sample degradation was observed in the immersed tissue samples. Raman spectra were pre-processed using MATLAB 2019b (MathWorks, Mass., U.S.A.) to remove cosmic rays, and relative intensity correction was done by utilizing the measurements made on a fluorescence standard (SRM 2241, NIST, U.S.A.) [77] twice within the testing period. To further reduce systematic error, measurement of polystyrene standard samples was conducted the beginning of each measurement period to ensure recorded intensities were in a consistent range. The background was removed using the sensitive non-linear iterative peak-clipping (SNIP) baseline subtraction algorithm $[76,78]$. The SNIP technique iteratively determines a baseline to subtract from the spectrum by finding the minimum between a given point and the average value of the outer edges of a window centered on that point. Since the background of the Raman spectrum through-the-surface changes with depth, the window width for SNIP was correspondingly altered to optimize the match with equivalent cross-section Raman spectrum. This window size (CCD pixels) was then fixed for a given depth across all samples: 135 at the surface and $100 \mu \mathrm{m}$; and 105, 75 and 85 at $25 \%, 50-75 \%$ and $100 \%$ depth respectively. All spectra were normalized using vector normalization to facilitate comparison between spectra recorded with the same experimental parameters but slightly different conditions.

Based on other studies using Raman spectroscopy in cartilage $[16,21,34,39]$ tentative wave number assignments were given as per Table 1.1. 


\subsubsection{MCR composition determination}

The goal of MCR is to determine the contributions of sub-components in a mixture to the overall sample signal through Eq.(6) [46]. In this equation, $D$ represents a matrix of the combined mixture spectra, $C$ represents a matrix of the relative concentrations for the corresponding matrix of individual pure reference spectra $S$, and $M_{\text {Error }}$ represents the remaining error in the fit [46]. Sub-component reference spectra, of known dilution, can then be used in a correlation curve to find the concentration of analyte in a mixture's Raman spectrum.

The pure signal contributions in cartilage were assumed to be composed of collagen II, GAG, and distilled water, similar to previously mentioned studies [21]. Laboratory grade collagen II was obtained from chicken sternal cartilage (Sigma-Aldrich), while chondroitin sulfate salt from bovine articular joints was used for reference GAG spectrum (Sigma-Aldrich). MCR deconvolutions of cartilage were fit between the wavenumbers $400-1800 \mathrm{~cm}^{-1}$ due to the high molecular specificity in this range $[20,21]$. MCR was conducted separately on each specimen and clearing stage to improve sample specific reference spectra identification. This was also to minimize the effects organization-dependent Raman band intensities may have if the deconvolutions contained both cross-section and through-the-surface spectra. Thus, it was assumed that variations in collagen spectra were due to concentration and no attempt was made to account for fibril orientation. In all through-the-surface measurements the water spectrum was fixed to the reference to prevent unwanted manipulations by the MCR-ALS algorithm.

\subsubsection{Concentration scaling factor}

Once the percent signal contribution curves with depth for each MCR sub-component were obtained from cross-sections, they were normalized with respect to their maximum value. It was assumed that the absolute concentration of a component was proportional to the relative concentration $C$, in Eq.(6). 
Using the measured wet and dry specimen weight, Eq.(22) and (23) were used to find a scaling factor for the normalized component curves. Multiplying the normalized curves by this scaling factor yielded the estimated concentrations of each sub-component at respective depths.

$$
\begin{gathered}
\text { Wet mass }- \text { Dry mass }=\text { Water mass }=\int_{0}^{L} \text { Area } \cdot\left[b_{\text {liquid }} \cdot \rho_{\mathrm{H}_{2} O}(l)\right] d l \\
\text { Drymass }=\int_{0}^{L} \text { Area } \cdot\left\{b_{\text {solid }} \cdot\left[\rho_{\text {Coll II }}(l)+\rho_{G A G}(l)\right]\right\} d l
\end{gathered}
$$

In these equations Area represents the caliper-measured cross-sectional area of each sample, $\rho$ represents the normalized $C$ signal curves from MCR in Eq.(6), $L$ represents the thickness of the sample, and $b$ represents the respective scaling factor for the sub-components. In Eq.(23) the maximum GAG and collagen II concentrations are assumed equal since their normalized curves are both multiplied by the same scaling factor. This was deemed acceptable due to the observation that peak sub-component concentrations roughly coincided in related studies with cartilage [21]. When using this assumption it was found that collagen II accounted for just over half of the dry weight, which has also been documented $[2,4]$. 


\subsection{Results}

\subsubsection{Raman spectra through the surface}

a

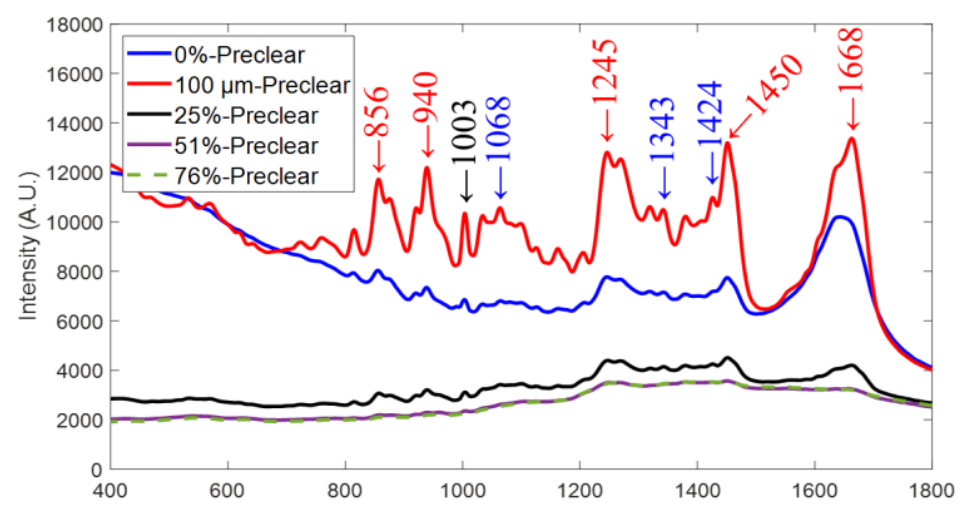

$\mathrm{b}$

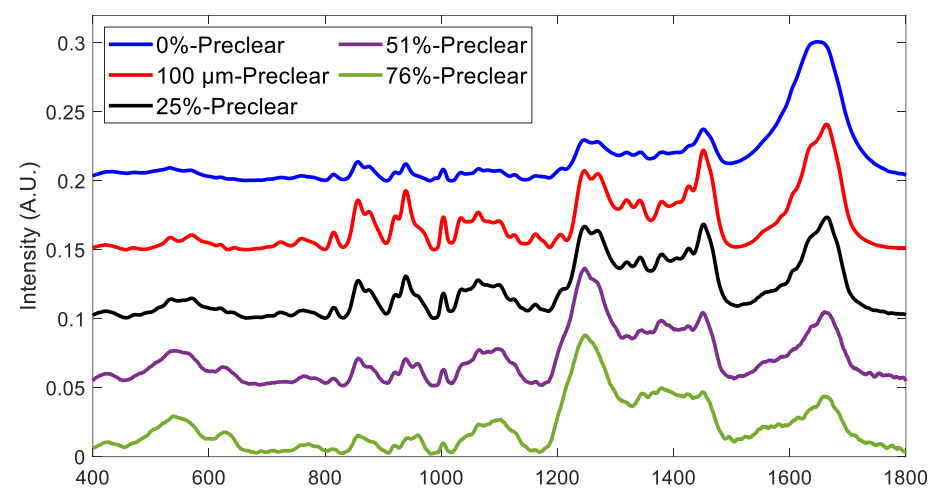

C

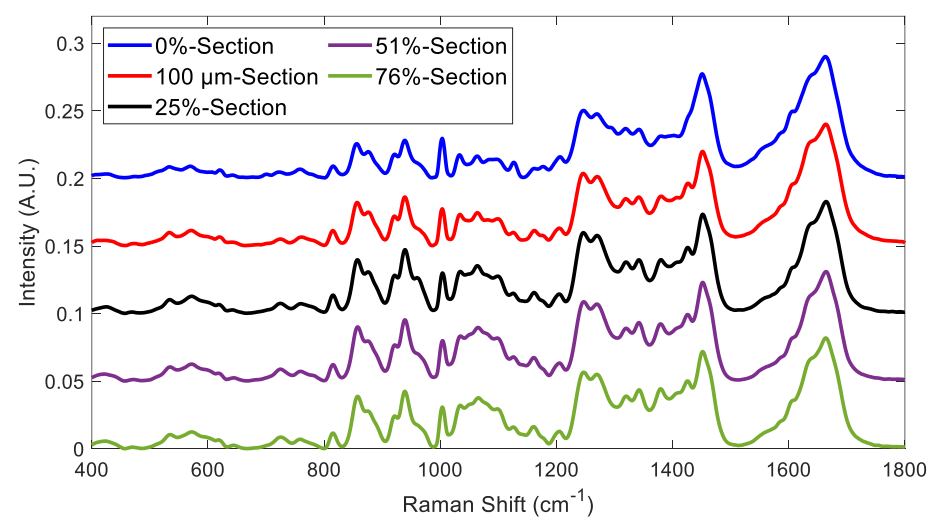

Figure 2.3: (a) Raw preclear Raman spectra through-the-surface with no background removal or normalization. (b) Offset pre-processed preclear spectra for treated sample through-the-surface. (c) Offset pre-processed cross-section spectra for comparison. Red labeled wavenumbers specify peaks most associated with collagen II, blue with GAG, and black for shared or other sub-components. 
Raw spectra (mean of 15 acquisitions) from the preclear through-the-surface measurements for a treated sample are shown in Figure 2.3(a) to show the effects of attenuation with depth. The preprocessed and normalized preclear and cross-section measurements for the same sample are shown in Figure 2.3(b) and (c), respectively. These normalized spectra illustrate the measurements used as inputs to the MCR-ALS algorithm.

\subsubsection{Cross-section composition from MCR}

The results of MCR analysis for an individual control sample cross-section, representative of all crosssectioned samples (treated and control), are shown in Figure 2.4(a,b,c) illustrating the difference between MCR deconvolved sub-components and reference spectra. The average explained variance of each deconvolved sub-component in cross-sections with respect to reference spectra was $R^{2}=95.45 \pm$ $0.38 \%(\operatorname{LoF}(\%)=29.81 \pm 1.24 \%)$ for water, $R^{2}=99.78 \pm 0.10 \%(\operatorname{LoF}(\%)=6.55 \pm 1.50 \%)$ for $G A G$, and $R^{2}=$ $97.75 \pm 0.42 \%(\operatorname{LoF}(\%)=21.09 \pm 1.95 \%)$ for collagen across all samples. After weighing samples both wet and dry, water was found to make up approximately $77.2 \pm 2.4 \%$ of the wet cartilage mass. Using sample volumes, the average tissue density of all samples was found to be $1.17 \pm 0.20 \mathrm{~g} / \mathrm{mL}$.

The $\rho$ curves obtained from cross-sections for each sub-component, multiplied by their respective scaling factors in Eq.(22) and (23), yielded the plots shown in Figure 2.4(d,e,f) for treated and control samples. Using the average density, the percent wet weight was also determined for each component based on concentration. Collagen remained at a relatively constant percentage of the average wet weight $(12.2 \pm 4.5 \%)$. The GAG content increased from $1.3 \pm 1.0 \%$ near the surface to $12.0 \pm 3.0 \%$ at $75 \%$ of the total thickness. The average calculated concentration of water was $103.8 \pm 15.8 \%$ of wet weight near the bearing surface, and then remained relatively constant at $70.7 \pm 28.4 \%$ through the remaining depth short of the bone surface. These uncertainty values are assumed only statistical in 
nature due to the constant immersion of samples (Section 2.3.2), and low tissue degradation over the testing period.

a
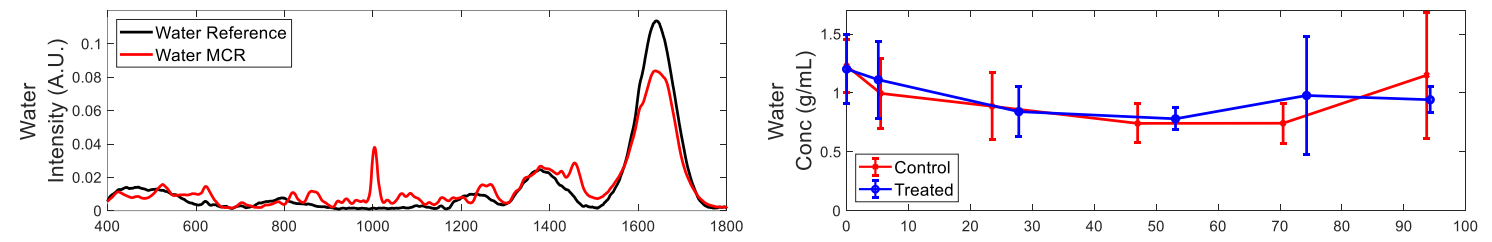

b
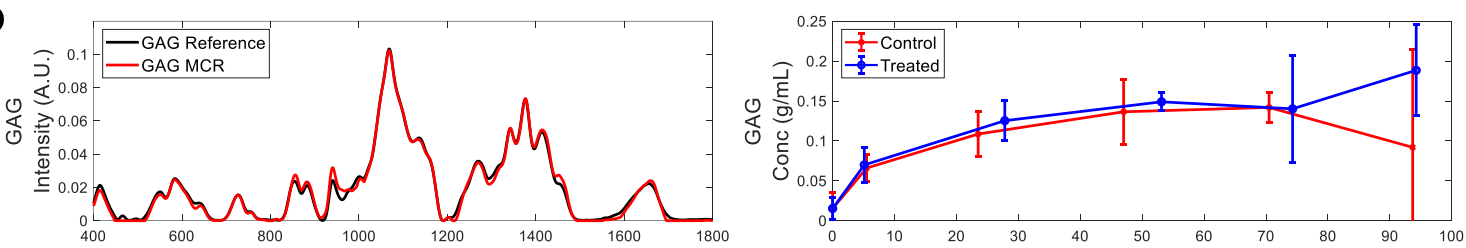

$\mathrm{C}$
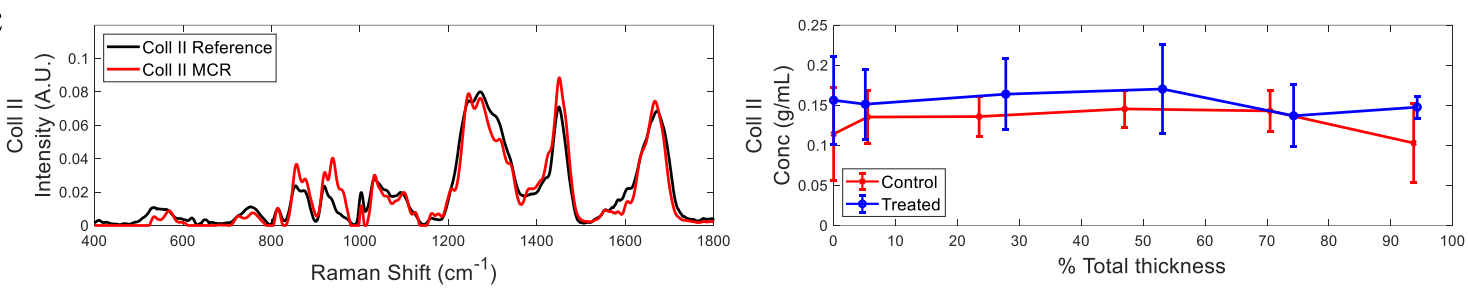

e

Figure 2.4: Reference vs. MCR deconvolved sub-component spectra for control sample through crosssection for (a) Water, (b) GAG, (c) Collagen II. Control and treated sample sub-component concentrations based on normalized MCR distribution curves through the cross-section for (d) Water, (e) GAG, (f) Collagen II. Error bars represent the $95 \%$ C.I. Depth measurements within a $10 \%$ thickness range were averaged into one depth based on negligible change in properties.

\subsubsection{Composition through-the-surface and clearing effects}

To find the depth to which MCR could accurately determine composition the contribution of each subcomponent to the sum of the three (Water, GAG, Collagen II) was evaluated. As can be seen in Figure 2.5(a-f), when comparing surface measurement stages to the cross-section standard, both treated $(a, c, e)$ and control $(b, d, f)$ sample signal begin to deviate after a depth of $25 \%$ of the total thickness $(0.48$ $\pm 0.13 \mathrm{~mm}$ ). Beyond this depth the MCR deconvolution begins to attribute the water contribution to collagen due to the signal attenuation. GAG content also does not reach its maximum at $75 \%$ of the total thickness due to attenuation. 
a

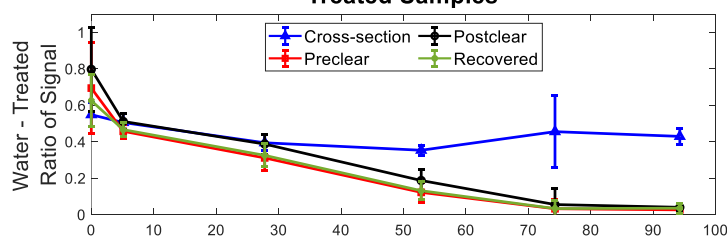

$\mathrm{C}$

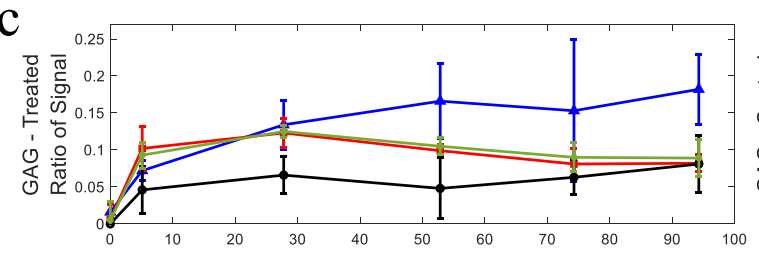

e

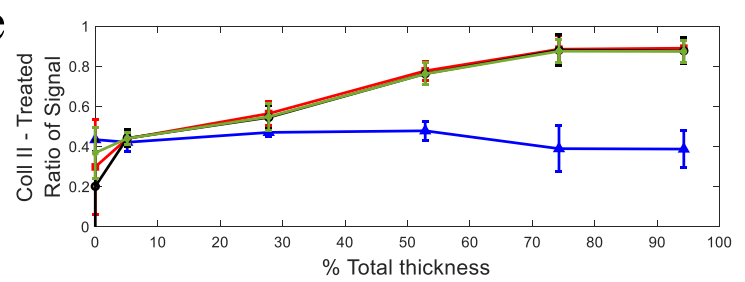

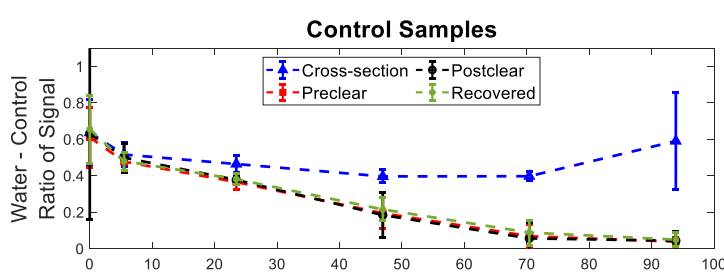
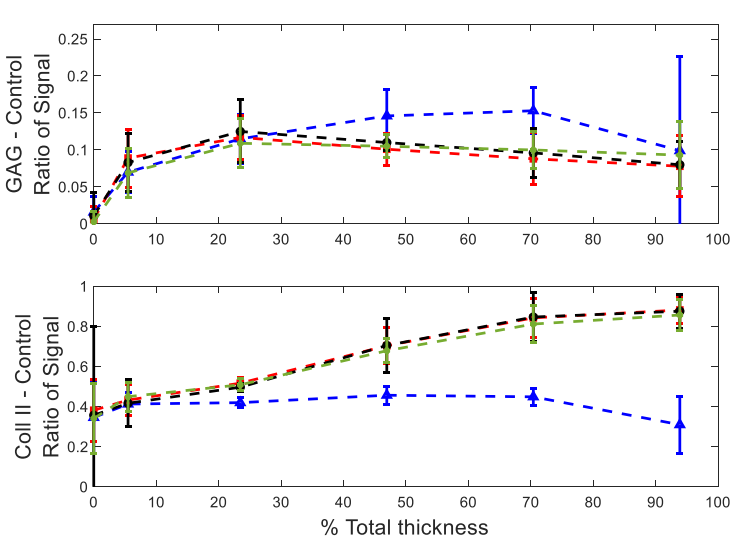

b

d

Figure 2.5: MCR deconvolved ratio of cartilage sub-component contributions to the sum of the main three molecules for (a) Water - Treated Samples, (b) Water - Control Samples, (c) GAG - Treated Samples, (d) GAG - Control Samples, (e) Collagen II - Treated Samples, and (f) Collagen II - Control Samples. Error bars represent the $95 \%$ C.I. Depth measurements within a $10 \%$ thickness range were averaged into one depth based on negligible change in properties.

Deconvolution of distilled water, GAG, and Collagen II in cross-sections yielded $R^{2}=96.12 \pm 0.43 \%$ $(\operatorname{LoF}(\%)=19.68 \pm 1.10 \%)$ in treated samples and $R^{2}=96.25 \pm 0.53 \%(\operatorname{LoF}(\%)=19.35 \pm 1.34 \%)$ in control samples for explained variance in cartilage spectrum. Due to the fixed water spectrum, throughthe-surface measurements had lower explained variance values of $R^{2}=91.71 \pm 0.46 \%$ (LoF(\%) $=28.79 \pm$ $0.79 \%)$ in treated samples and $R^{2}=92.02 \pm 0.67 \%(\operatorname{LoF}(\%)=28.12 \pm 0.82 \%)$ in control samples.

Figure 2.6(a) shows an overlay of the reference OCA spectra and cartilage at a depth of $100 \mu \mathrm{m}$. Dominant OCA peaks are present at $1003 \mathrm{~cm}^{-1}$ from symmetric C-N stretching in urea [79], $628 \mathrm{~cm}^{-1}$ from D-(-)-fructose $\mathrm{C}-\mathrm{C}-\mathrm{O}_{5}$ groups, and $524 \mathrm{~cm}^{-1}$ from the pyranose ring in D-(-)-fructose [80] among others. Figure 2.6(b) shows the postclear stage MCR deconvolutions, illustrating where OCA peaks coincide with other sub-components. OCA concentrations determined from MCR were sample dependent, and a reduced sample thickness did not necessarily improve penetration. 
a

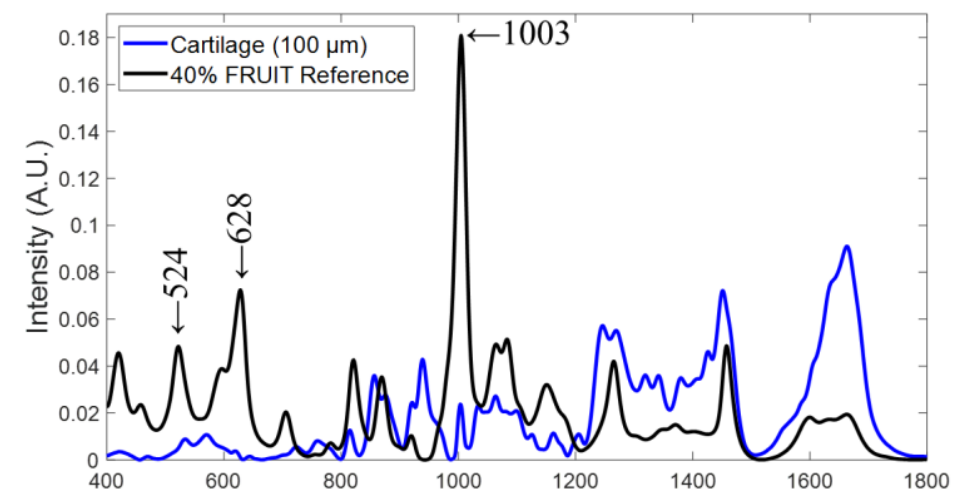

$\mathrm{b}$

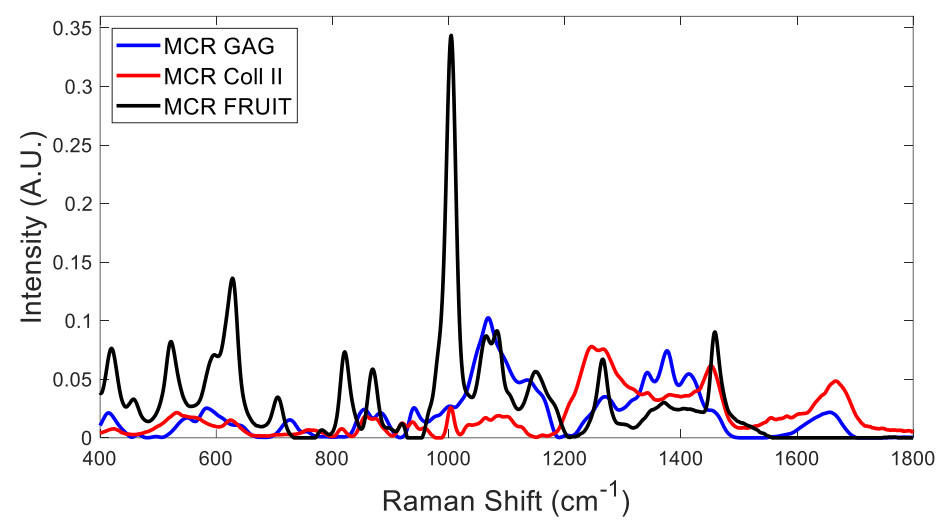

Figure 2.6: (a) Reference $40 \%$ FRUIT OCA Raman spectrum alongside cartilage spectrum at $100 \mu \mathrm{m}$ through-the-surface, (b) MCR deconvolved sub-components from postclear stage of a treated sample. Labeled wavenumbers represent OCA-related peaks.

\subsection{Discussion}

Raman spectroscopy-based MCR measurements of cartilage through-the-surface can reliably predict sub-component contributions up to a depth of approximately $0.5 \mathrm{~mm}$. This represents $25 \%$ of the cartilage thickness in this study. The clearing solution used in this study does not appear to have any benefit in regards to increasing this depth of resolution. This is apparent in Figure $2.5(\mathrm{a}, \mathrm{c}, \mathrm{e})$ which shows that the postclear stage follows similar trends as the preclear and recovered stages. GAG trends in Figure 2.5(c,d) for through-the-surface measurements suffer less from attenuation effects than the other sub-components in non-OCA treated stages. The discrepancy between the trends for the postclear stage and the untreated stages in Figure 2.5(c), is due to the OCA masking some of the GAG signal. This 
spectral overlap with the overall cartilage signal and deconvolved sub-components is seen in Figure 2.6(a) and (b) respectively. Based on the postclear spectra from all treated samples the OCA appears to penetrate much of the cartilage, with OCA-related peaks being present up to $75 \%$ of the total thickness (results not shown). In contrast, MCR deconvolution predicted OCA contributions to the overall signal decrease from $10.4 \pm 15.0 \%$ at the surface to $2.6 \pm 3.3 \%$ at a depth of $50 \%$ of the total thickness. This difference is likely due to a combination of the MCR-ALS algorithm attributing features of the OCA spectra to other sub-components, and the decreased signal to noise ratio at depth.

As seen in Figure 2.4 and Figure 2.5, measurement variance was much larger at the bearing and bone surfaces at $0 \%$ and $95 \%$ total thickness, respectively. This was largely attributed to steep composition gradients in these two regions $[4,20]$, and error in measurement position. The difference between the designated surface height and the maximum laser focal point could deviate up to $\pm 29 \mu \mathrm{m}$ due to the slope of the bearing surface in some samples, and reference wire placement from measurement location. This discrepancy was shown to have the greatest effect at the bearing surface interface with the PBS bath. Similarly, the tissue-bone interface was sensitive to any small measurement position errors incurred from the original estimated thickness values. Caliper measurement error also likely led to a portion of the variance in concentration values due to its use in area measurements (Section 2.3.7). In order to reduce unwanted manipulations of the water spectrum in MCR deconvolution, all throughthe-surface measurements had the water spectrum fixed to the reference. If not constrained, the MCRALS algorithm would manipulate the water spectrum to either incorporate Raman bands from the OCA, or account for attenuation effects due to its low molecular specificity in the $400-1800 \mathrm{~cm}^{-1}$ measurement range. These manipulations can be seen in the unconstrained cross-section water spectra in Figure 2.4(a) which incorporates a large Phenylalanine peak at $1003 \mathrm{~cm}^{-1}$. This constraint had the effect of lowering the explained variance values for the stages, but yielded more reasonable signal contributions. This highlights the need for a potential clearing agent to provide adequate scattering 
reduction to minimize the ALS curve fitting error associated with having an additional degree of freedom in the total number of sub-components.

Each component concentration estimation made from MCR shows a relatively large distribution. This demonstrates the variability in separate samples - even from the same joint surface. This high amount of variability should be expected in most biological samples and appears to be a common aspect of Raman measurements in cartilage $[20,21,81]$. Apart from this characteristic the MCR deconvolved spectra were not expected to match perfectly. Also present in the tissue are chondrocytes, hyaluronic acid, multiple collagen types, and other proteins in minor quantities. However, MCR explained $96 \%$ of the variance in cross-section measurements and $91 \%$ in through-the-surface measurements when assuming water, collagen, and GAG components only. Collectively the minor components are assumed to make up a majority of the remaining unexplained spectral variance [21].

The sectioned GAG spectral contribution trend in Figure 2.4(e) agrees well with past studies conducted on bovine cartilage $[20,21]$. However, the relatively constant collagen contribution with depth appears to follow the trend in the study by Bergholt et al.[20] more closely. The GAG concentrations found also align with studies using $\mu C T$ and $\mu \mathrm{MRI}$ up to $0.5 \mathrm{~mm}$ in cartilage from dogs [82, 83]. Using MCR only within the $400-1800 \mathrm{~cm}^{-1}$ range led to similar drawbacks also noted in previous studies specifically for water [21]. The low spectral specificity of water in this range causes a drop in signal contribution with depth. This is likely the source of the high water concentrations seen near the surface in Figure 2.4(d). Ideally a higher wave number range incorporating $3200-3650 \mathrm{~cm}^{-1}$, taking advantage of the $\mathrm{O}-\mathrm{H}$ stretching vibration, would be used to determine water content [21]. As noted in other studies spectral measurements in this higher range also offer access to quantifying matrix bound water molecules, not just the water present in the interstitial fluid [84]. In spite of this drawback the water content agrees with previous assay studies done by Oswald et al.[22] through the middle zone of the tissue. 
Future studies will need to assess the feasibility of Raman-based composition determination in-situ or in-vivo, particularly for applications such as arthroscopic surgeries [33]. Further work with clearing duration and alternative OCAs, specifically those with less spectral overlap of important collagen and GAG features, could improve through-the-surface Raman spectroscopic measurements of cartilage. Additionally, attenuation was noted to have a reduced effect on GAG content determined through-thesurface in comparison to other sub-components (Figure 2.5). This suggests potential use of a scaling factor for through-the-surface measurements to match cross-section distributions in future studies.

\subsection{Conclusion}

This proof-of-concept study shows Raman-based MCR determination of cartilage sub-component distributions through-the-surface can be accurately quantified up to a depth of $0.5 \mathrm{~mm}$. Use of a fructose-based OCA did not increase this depth. For an OCA to increase this depth of resolution it must reduce scattering enough to overcome the inherent error introduced by adding an additional degree of freedom to the MCR-ALS spectra fitting process. Future work with clearing duration and alternative clearing agents, particularly those with less spectral overlap of important collagen and GAG features, could improve through-the-surface Raman spectroscopic measurements of cartilage. The nondestructive means of composition determination through-the-surface shown in this study is of particular interest due to its potential use in-situ or in-vivo. A specific application would be in arthroscopic joint surgeries [33] for the evaluation of compositional changes in osteoarthritis development, characterized by progressive GAG loss starting at the surface [4]. 


\section{Chapter 3: \\ Raman spectroscopy-based MCR for applying depth-dependent material properties to articular cartilage FE models}

This Chapter will be submitted to the Journal of Osteoarthritis and Cartilage as:

Mason D.R., Murugkar S., Speirs A.D., "Raman spectroscopy-based MCR for applying depth-dependent material properties to articular cartilage FE models".

This chapter represents a version of this manuscript, adapted for formatting within the thesis and based on examiner recommendations. 


\section{Raman spectroscopy-based MCR for applying depth-dependent material properties to articular cartilage FE models}

\subsection{Abstract}

Objective

Sub-component distributions in the composition of cartilage are known to play a key role in its mechanical response. We aim to highlight improved tissue response in depth-dependent finite element (FE) models created using Raman spectroscopy compared to standard models. The feasibility of using Raman measurements through-the-surface for this purpose is also assessed for potential use in-situ or in-vivo.

\section{Design}

Distributions of water, collagen II, and glycosaminoglycan (GAG) were determined through-the-surface in cartilage samples $(n=11)$ before, during, and after treatment with an optical clearing agent (OCA) using Raman-based multivariate curve resolution (MCR). Cross-section Raman spectra were then acquired to use as standards. Stress-relaxation indentation testing was also completed at the beginning and end of the testing regime while the reaction force was recorded. Depth-dependent material property FE models were then created and optimized in FEBio using cross-section data to match experimental results. Through-the-surface measurement-based models were also produced using a regressed scaling line to match cross-section results. Comparisons were made against equivalent homogeneous distribution models. 
Results

Depth-dependent models showed an average improvement in predicted reaction force of $18 \%$ over homogeneous models. Through-the-surface measurement-based models had a difference in predicted reaction force from cross-section counterparts of approximately 4\%. Depth-dependent models improved fluid load support an average of 3.6\% over homogeneous models.

\section{Conclusion}

Raman spectroscopy is a useful tool for applying material property distributions in FE models of cartilage and improves predicted tissue response. Through-the-surface Raman measurements are also of potential use in-situ or in-vivo, where they could be employed in modeling of developing osteoarthritis.

\subsection{Introduction}

Articular cartilage serves as a wear resistant, and nearly friction-free, bearing surface in diarthrodial joints $[1,2]$. To achieve this the tissue relies on a unique depth-dependent and heterogeneous structural composition. Measuring subtle biochemical changes to this composition is key to assessing the etiology and development of joint diseases such as osteoarthritis (OA). Raman spectroscopy has shown the capability of not only discerning minute changes to this structure [24, 34, 35], but also determining macro-scale composition and organization in tissue cross-sections [20,21]. Use of various imaging techniques for applying this structural hierarchy to FE modeling of cartilage $[56,57]$ has proven invaluable in understanding the mechanical contributions of each sub-component. To date, use of Raman spectroscopy in cartilage cross-sections for this same purpose has not been published. Moreover, if FE models using non-destructive Raman measurements made through-the-surface could be shown to match cross-section equivalents; data obtained via spectroscopes used in-vivo [33] could be of practical application for simulation purposes. 
Articular cartilage consists predominantly of an extra cellular matrix (ECM) scaffold, with interstitial fluid filling the voids. The main components of the ECM are type II collagen and proteoglycan, with a sparse population of chondrocyte cells that produce ECM components [1, 2]. Articular cartilage is traditionally considered to consist of three zones: The superficial zone (top 10-20\% of tissue) where collagen fibrils are aligned parallel to the bearing surface, the middle zone (next $40-60 \%$ of tissue) where collagen fibrils are more randomly oriented, and the deep zone (remaining $20-30 \%$ of tissue) where collagen fibrils are mainly oriented perpendicular to the bearing surface and insert into the subchondral bone below [1, 2]. Throughout these zones the proteoglycan content gradually increases with depth [20,21]. Water content reaches its maximum near the bearing surface before dropping and maintaining approximately $75 \%$ of the wet mass in the middle and deep zones [22]. This organization of sub-components gives the tissue anisotropic and non-uniform biomechanical properties which differ in tension and compression [61].

To capture the tension-compression nonlinearity in FE modeling of articular cartilage many studies have simulated the tissue as a biphasic poroviscoelastic (BPVE) material with fibril reinforcement, or as close equivalents [56-59, 61-63, 68, 69]. Many of these studies have also noted the importance of accounting for the depth-dependent structure in models to obtain an accurate tissue response. In particular the enhanced interstitial fluid pressurization, and associated fluid load support, generated in these depthdependent models has been routinely reported due to its importance in reducing surface friction and wear in cartilage [56, 58-60].

Recently Raman spectroscopy, coupled with the use of MCR, has been shown to accurately predict subcomponent concentrations in native and engineered cartilage cross-sections [20, 21]. This depth-wise composition information from label-free Raman spectroscopy could be used to develop more accurate FE simulations of cartilage. 
Studies using Raman-based compositional information of cartilage have so far been restricted to use in tissue cross-sections. This is a destructive assessment of the tissue and is impractical for use in-situ or invivo. Ideally, composition measurements could be made through-the-surface in a non-destructive manner. However, optical attenuation with depth severely impacts Raman spectroscopic measurements through-the-surface in turbid tissues. This optical attenuation is a side effect of the heterogeneous composition of cartilage [49]. Studies have reported that the use of optical clearing agents (OCA) can reduce scattering in the tissue without affecting morphology. Neu et al.[53] used this technique for articular cartilage up to a depth of $2.5 \mathrm{~mm}$ with the use of a fructose-based OCA. Another study [55] showed that optical clearing of cartilage with iohexol could enable visibility of the cartilage-subchondral bone interface with optical coherence tomography in as little as 15 minutes.

The goal of this study is to assess if Raman spectroscopy-based MCR measurements of cartilage subcomponent distributions, made via cross-sections, can improve FE modeling of the tissue. This is assessed by conducting stress-relaxation indentation tests on samples $(n=11)$ and creating FE models with depth-dependent material properties, based on Raman measurements, and optimized to match the experimental results. The depth-dependent models are then evaluated alongside homogeneous distribution models to compare predicted material responses. Models based on Raman measurements made through-the-surface were also investigated to assess potential use in-situ or in-vivo.

An OCA similar to the fructose-based FRUIT[74] was utilised in this study in an attempt to reduce scattering for measurements made through-the-surface. Its effects, along with Raman results, are outlined in a report made in parallel to this study in Chapter 2 . 


\subsection{Methods}

\subsubsection{Specimen preparation}

Skeletally mature porcine knee joints were locally sourced from an abattoir up to 48 hours after slaughter. Cylindrical cartilage plugs $(\varnothing 10 \mathrm{~mm} \times 6 \mathrm{~mm})$ from the trochlear groove or patella were then excised $(n=12)$ from three joints and frozen in a 1xPBS solution. Each sample consisted of up to $2.5 \mathrm{~mm}$ of articular cartilage with the remaining height consisting of the subchondral bone below. Plugs frozen together and used in testing groups were mixed from the three joints to provide more variability.

\subsubsection{Mechanical loading and testing regime}

Upon thawing for one hour the thickness of the cartilage samples was initially estimated using a Vernier caliper (0.2 $\mathrm{mm}$ resolution). The samples were then placed in a stock 1xPBS solution supplemented with an EDTA-free protease inhibitor (SIGMAFAST ${ }^{\mathrm{TM}}$; Sigma-Aldrich Corp., Mo., U.S.A.) below a $\varnothing 1.6 \mathrm{~mm}$ impermeable flat indenter in a custom built device [69] (see Figure 3.1(a)). After giving the tissue 25 minutes to recover in this bath the indenter was moved downwards until a reaction force of $20 \mathrm{mN}$ was recorded. This position was deemed the initial contact of the articulating surface [62], and was held for 15 minutes to ensure an initial equilibrium state within the cartilage. A stress-relaxation indentation test was then performed by displacing the indenter to $7 \%$ of the tissue thickness, as estimated by caliper measurement, at a speed of $33 \mu \mathrm{m} / \mathrm{s}$. The indenter was held in the extended position for 30 minutes, by which time the reaction force reached a state of equilibrium. The reaction force was recorded from the start of the experiment. The indenter diameter was chosen based on previous research showing a

negligible change in tissue response when $R_{\text {cartilage }}>4 R_{\text {indenter }}$ [85]. Based on this same study it was assumed curvature at the cartilage-bone interface had a negligible effect on tissue response. 
Each sample $(n=5)$ underwent a four-day testing regime described in Chapter 2. Briefly, test specimens underwent Raman measurements before (preclear stage), during (postclear stage), and after (recovered stage) treatment with a fructose-based OCA. Raman measurements were then used with MCR to map depth-wise sub-component distributions through-the-surface. Samples were then cross-sectioned and Raman-based MCR measurements repeated to use as standards. Specimens underwent loading prior to the preclear and recovered stages to quantify time related changes in mechanical response. These samples are referred to as "Treated". Control samples $(n=6)$ underwent the same testing regime but were not treated with the OCA; mechanical tests were performed at equivalent time points. Results are reported as mean $\pm 95 \%$ C.I. unless otherwise stated.

\subsubsection{Thickness test}

To accurately obtain the sample thickness a needle was pushed through the cartilage surface while the reaction force was recorded. This was done using the same device for indentation, seen in Figure 3.1(a), with the indenter replaced by a 22-gauge needle. The tissue surface was identified once the reaction force exceeded $5 \mathrm{mN}$. The bone surface was identified by a sudden large change in the reaction force slope with respect to distance. The average sample thickness was $1.9 \pm 0.5 \mathrm{~mm}$.

Data processing was completed using custom scripts created in MATLAB 2019b (MathWorks, Mass., U.S.A.). 
a

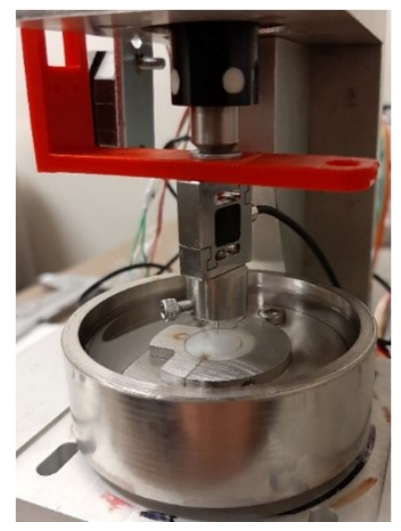

b

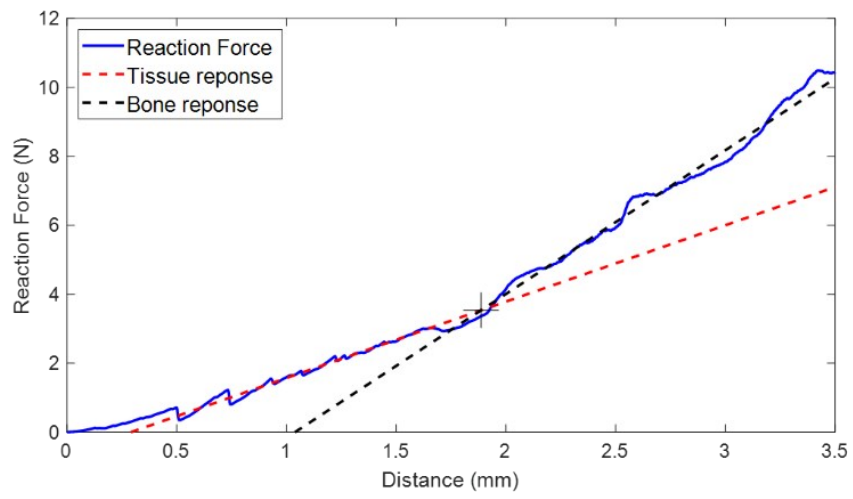

Figure 3.1: (a) Cartilage sample undergoing thickness test using custom-built device. (b) Typical reaction force plot for thickness test where (+) indicates tissue/bone intersection chosen as thickness value.

\subsubsection{Finite Element Model}

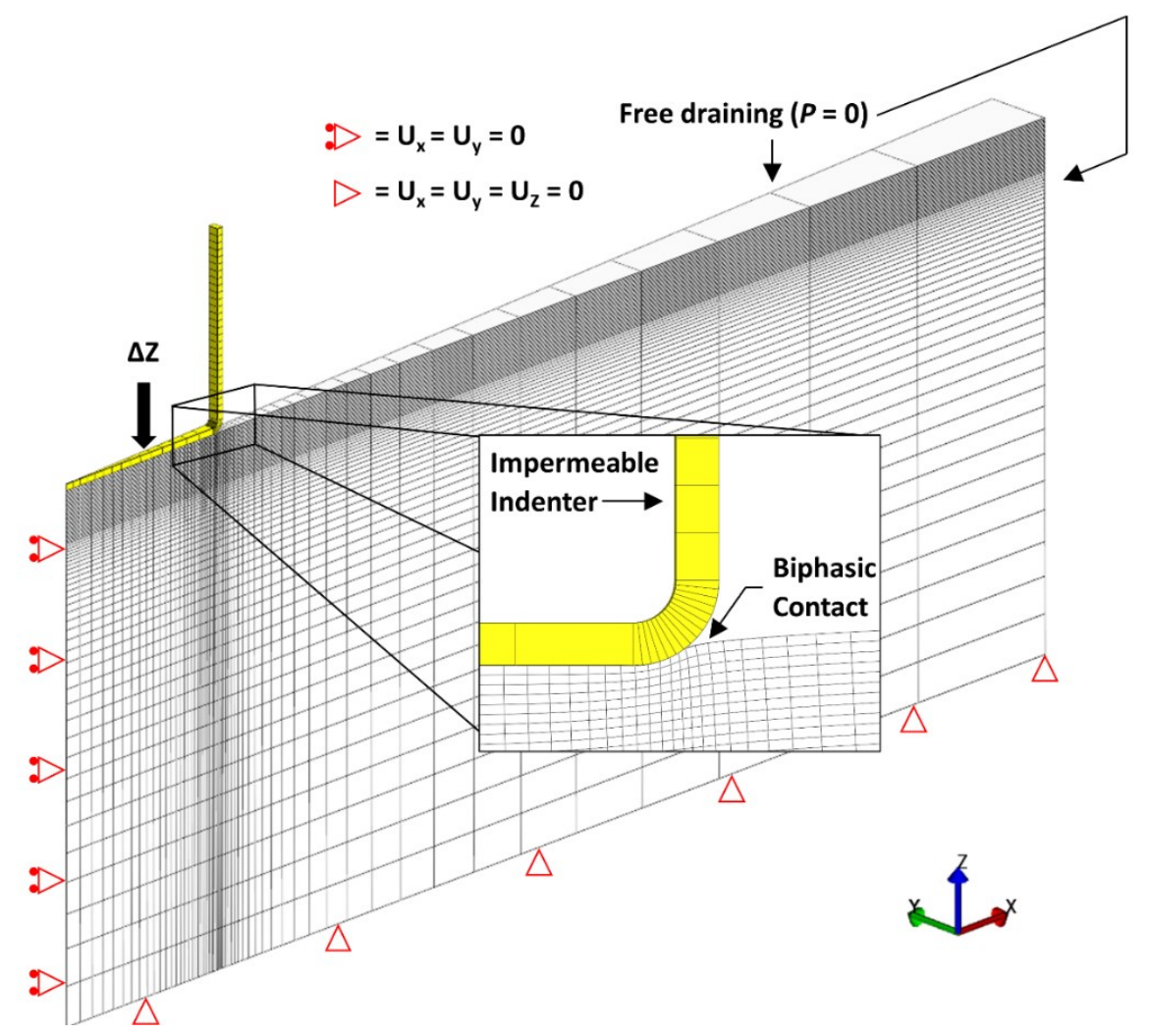

Figure 3.2: Finite element wedge model and boundary constraints used for stress-relaxation indentation testing of articular cartilage. The boundary conditions applied to the wedge reproduce the axisymmetric nature of the test. Yellow material represents the impermeable indenter, white material represents the BPVE cartilage material. 
The cartilage indentation tests were modeled as $3^{\circ}$ axisymmetric wedges (as seen in Figure 3.2 ) created within FEBio 2.9, and consisted of 4200 predominantly linear hexahedral elements. FEBio does not support two dimensional elements, so axisymmetry was achieved by selecting appropriate boundary conditions on a wedge-shaped volume. Modeling the tissue as a wedge with suitable boundary conditions is a common means of simulating axisymmetric conditions in finite element solvers, producing similar results to $2 \mathrm{D}$ modeling with axisymmetric elements $[62,69,73,86]$. The mesh was chosen based on refinements where a doubling of elements was found to change the maximum stress by less than $5 \%$. This criterion was selected based off of previous studies using the same limit when conducting similar FE modeling of cartilage [69]. Additional information on the mesh sensitivity study is provided in Appendix B. One model exceeded the $7 \%$ strain value due to caliper estimation error and was further refined to 5600 elements to meet this criterion.

\subsubsection{Constitutive model}

To account for the solid structure of the articular cartilage in FE modeling a continuous ellipsoidal fiber distribution was implemented in conjunction with a supporting ground substance using FEBio 2.9. This constitutive model was chosen based on reported improved accuracy over mutually orthogonal fiber bundle and cone-wise linear elastic models, particularly in the flow-independent phase of loading [63]. The constitutive equations are outlined in Section 1.10, with details provided for the main parameters as follows.

The strain energy density function ( $\Psi$ ) for the fibers is given by Eq. (13) where $\beta \geq 2$ and $I_{n} \geq 1$ [63]. $\xi\left(n^{r}\right)$ and $\beta\left(n^{r}\right)$ are variable material parameters along the fiber orientation with the former being related to the fiber tensile modulus in units of force per unit area, and the latter being unitless $[63,73]$. $\xi_{1-3}$ and $\beta_{1-3}$ represent the corresponding material parameters along their respective ellipsoid semi- 
axes $(1=\mathrm{X}, 2=\mathrm{Y}, 3=\mathrm{Z})$ in the local coordinate system. When $\beta=2$ this results in a jump at the strain origin in elasticity modulus for fibers transitioning to tension at small deformations [63]. The ground substance supporting the continuous fiber distribution, aimed at accounting for the proteoglycans, is modeled as a compressible neo-Hookean hyperelastic material. The response of this material is governed by an elastic modulus $\left(E_{m}\right)$ and a Poisson's ratio. Fluid flow through the porous ECM is modelled with Darcy's law since the low permeability, on the order of $10^{-15} \mathrm{~m}^{4} / \mathrm{N} \cdot \mathrm{s}$ [15], results in low fluid flux and viscous shear forces. The response of this fluid phase is governed by the hydraulic permeability $(k)$.

\subsubsection{Material property distributions}

The FEBio plugin DataMap was augmented (Appendix F) to make the cartilage material properties not only depth dependent, but also a function of optimizable scaling factors. The superficial zone was assumed to make up the top $20 \%$ of tissue, followed by $50 \%$ and $30 \%$ for the middle and deep zones respectively [1]. Using the Raman-based MCR results from cross-sections, sample-specific normalized GAG content was mapped to the FE mesh as a function of depth with the adapted plugin. The modulus $\left(E_{m}\right)$ for the ground substance, representing the proteoglycan network, was assumed to be directly proportional to the GAG content. The $E_{m}$ at a specific depth was then scaled as $E_{m}(l)=\rho(l) \cdot E_{\text {scale }}$; where $\rho(l)$ represents the normalized GAG content determined from MCR as a function of the depth $l$, and $E_{\text {scale }}$ represents an optimizable scaling factor which is constant through the depth within an individual sample. The $E_{m}$ value at $100 \%$ of the thickness (tissue-bone interface) for all samples was assumed equal to the next deepest measurement. To account for collagen fibril orientation the fiber stiffness parameter at a specific depth was similarly multiplied by an optimizable scaling factor ( $\left.\xi_{\text {scale }}\right)$, but used a generalised depth-dependent distribution as fibril orientation was not directly measured. This generalized fiber stiffness parameter distribution in the transverse $(X-Y)$ plane was set to be 
maximum in the superficial zone and reduced to $50 \%$, and $33 \%$ through the middle and deep zones respectively. The fiber stiffness parameter in the axial (Z) direction followed the inverse of this trend. While the fiber tensile stiffness in the constitutive model used in this study is not linearly related to the strain-dependent $\xi$, it was deemed acceptable to vary it in this manner simply to demonstrate contrast between layers. Fiber stiffness was not varied based on collagen content determined from MCR as it was found to have a relatively constant distribution throughout the thickness. The unscaled material parameter distributions applied to an individual sample are shown in Figure 3.3. Material parameter transitions between data points were linear to improve solver stability. The scale parameters, $E_{\text {scale }}$ and $\xi_{\text {scale }}$, were estimated using the optimization procedure in FEBio, described below.
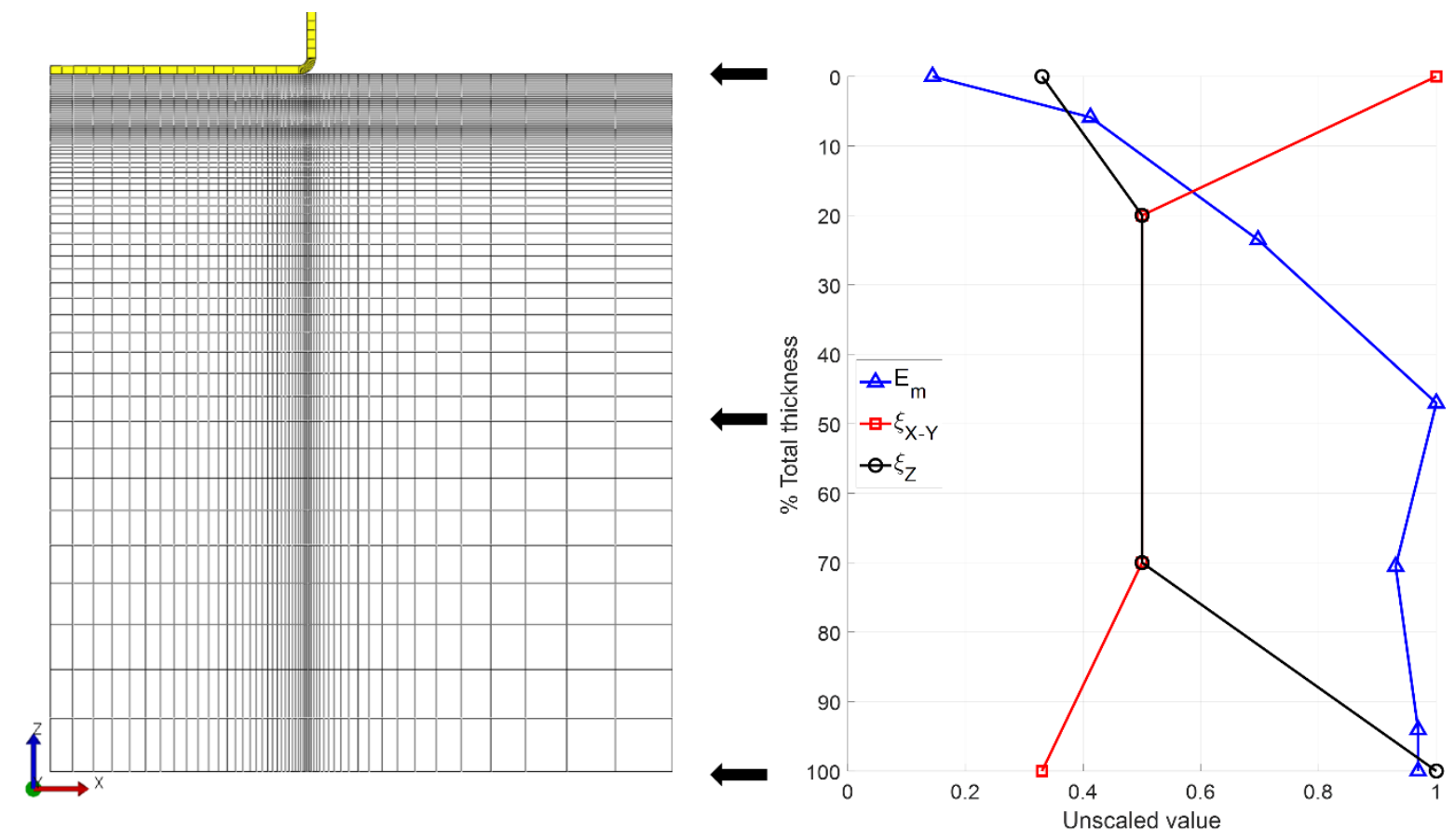

Figure 3.3: Unscaled material property distributions mapped to FE model mesh for an individual control sample. The absolute material parameters applied to the model were scaled by their respective values obtained by optimization as explained in the text. 
The Poisson's ratio in cartilage has been shown to approach zero when compressive strains exceed $4 \%$ [64]. This parameter has also been shown to be lowest in the superficial zone [67] which accounts for the greatest contribution to tissue response in surface-based indentation testing [62]. Therefore, to simplify models a constant Poisson's ratio of 0.03 was assigned to the ground substance in the tissue. Solid volume fractions for each sample were set constant and determined from the wet and dry weight of samples determined in Chapter 2. Permeability was also set constant with depth to simplify the models (preliminary evaluation of the effect of composition and depth-dependent permeability is presented in Appendix D). The exponential fiber parameter was fixed $(\beta=2)$ to enforce a jump in stress contribution across the strain origin when transitioning to tension.

For comparison with the depth-dependent models mentioned above, homogeneous models were created using the same constitutive relationships. In these models, material parameter distributions were uniform through the thickness. Each fiber stiffness parameter $\left(\xi_{X, Y, Z}\right)$ was set to have an unscaled distribution value of one (spherical distribution) through the entire thickness. The same was done for the ground substance modulus $E_{m}$. The material parameters in these models were also estimated using the optimization procedure in FEBio explained below.

\subsubsection{Boundary conditions}

The cartilage-bone interface was completely fixed from movement in the $X, Y$, and $Z$ directions, while the center axis of the cartilage plug was fixed in the $X$, and $Y$ directions as seen in Figure 3.2. The circumferential and bearing surfaces of the cartilage plug were free draining, except where there was contact with the indenter. The contact between the indenter and cartilage was modelled as a frictionless biphasic interface, ensuring impermeability in the contact region [73]. The $\varnothing 1.6 \mathrm{~mm}$ indenter incorporated a $0.05 \mathrm{~mm}$ radius fillet at the edge to improve solver stability. The indenter was 
modeled as a rigid body which descended a distance approximately $7 \%$ of the total thickness of the cartilage sample to reproduce the experimental conditions. The front face of the cartilage was constrained to inhibit movement in the $\mathrm{Y}$-direction, while the back face shared a tension-compression contact [73] with a symmetry plane enforced by a large penalty factor.

\subsubsection{Optimization scheme}

The material property scaling factors $E_{\text {scale }}$ and $\xi_{\text {scale }}$ along with tissue permeability were estimated using FEBio's built-in Levenberg-Marquardt based optimization algorithm (Eq. (21)) [73]. This minimizes the sum of squared difference between experimental and FE-predicted reaction force over the test duration. For the homogeneous models, the material parameters were estimated directly.

\subsubsection{Statistical analysis}

Paired t-tests were performed on the peak reaction force, and equilibrium response (average of last $10 \mathrm{~s}$ of indentation test), between the preclear and recovered loading stages of all samples to assess differences between the two time points. Unpaired t-tests were conducted on the percent difference values between these two loading stages (both peak and equilibrium response) across the two sample groups (treated and control). This was to assess differences in tissue response due to OCA treatment. Statistically significant findings were classified as $p<0.05$. 


\subsection{Results}

\subsubsection{Cross-section based model results}

As reported in Chapter 2 after weighing samples both wet and dry, water was found to make up approximately $77.2 \pm 2.4 \%$ of the wet cartilage mass. Optimized material parameters are presented in Table 3.1 for both the depth-dependent and homogenous models in treated and control samples. Zone based $E_{m}$ values in this table represent the average value at $0 \%, 50 \%$, and $100 \%$ tissue thickness respectively. The percent difference of the objective function value between the depth-dependent and homogenous models across all samples averaged $18.2 \pm 57.1 \%$ in favour of depth-dependence. All optimizations were conducted using reaction force data from the recovered stage loading. Variations in strain percentage occurred due to error in initial caliper thickness measurements, later confirmed using the thickness test (Section 3.3.3). The sensitivity of tissue response to variations in material parameters between samples are highlighted in Appendix C. Variations in homogeneous model material parameters show a relatively linear change in predicted material responses. This is demonstrated by treated samples 1, 2, and 4 which all have similar predicted material parameters in Table C.2 and responses in Table C.6. Control samples have a particularly wide ranging permeability as seen in Table 3.1, leading to larger variations in predicted material responses reported in Table C.5.

To assess if depth-dependent permeability could improve optimizations two preliminary models were also run using the FEBioStudio 1.0 .0 beta software package. These models were similar to their depthdependent counterparts, but permeability was scaled proportionally to the normalized water content determined from Raman-based MCR. These models produced optimization values with a difference of $2.3 \pm 0.9 \%$ from their constant permeability counterparts. Based on this result depth-dependent permeability was neglected in models (See Appendix D). 
Table 3.1: Material parameters for FE models determined from optimizations (DD = Depth-dependent model, $\mathrm{H}=$ Homogeneous model, SVF = Solid volume fraction, $\mathrm{SZ}=$ superficial zone, $\mathrm{MZ}$ = middle zone, DZ = Deep zone). Ranges represent the $95 \%$ confidence interval. All parameters listed were constrained to be positive.

\begin{tabular}{|c|c|c|c|c|}
\hline $\begin{array}{l}\text { Material } \\
\text { Parameter }\end{array}$ & Treated (DD) & Treated (H) & Control (DD) & Control (H) \\
\hline E-scale & $0.53 \pm 0.49$ & \multirow{4}{*}{$0.27 \pm 0.27$} & $0.30 \pm 0.36$ & \multirow{4}{*}{$0.16 \pm 0.19$} \\
\hline $\begin{array}{l}S Z-E_{m}(\mathrm{MPa}) \\
(0 \% \text { thickness) }\end{array}$ & $0.05 \pm 0.06$ & & $0.03 \pm 0.06$ & \\
\hline $\begin{array}{l}\mathrm{MZ}-\mathrm{E}_{\mathrm{m}}(\mathrm{MPa}) \\
\text { (50\% thickness) }\end{array}$ & $0.49 \pm 0.47$ & & $0.29 \pm 0.35$ & \\
\hline $\begin{array}{l}\mathrm{DZ}-\mathrm{E}_{\mathrm{m}}(\mathrm{MPa}) \\
\text { (100\% thickness) }\end{array}$ & $0.53 \pm 0.51$ & & $0.20 \pm 0.34$ & \\
\hline ל-scale & $0.87 \pm 0.13$ & $0.45 \pm 0.16$ & $0.67 \pm 0.41$ & $0.38 \pm 0.23$ \\
\hline $\begin{array}{l}\text { Permeability } \\
1 \times 10^{-15}\left(\mathrm{~m}^{4} / \mathrm{N} \cdot \mathrm{s}\right)\end{array}$ & $5.39 \pm 5.42$ & $5.27 \pm 5.32$ & $11.00 \pm 20.55$ & $12.78 \pm 22.18$ \\
\hline$\%$ strain & \multicolumn{2}{|c|}{$7.52 \pm 1.82$} & \multicolumn{2}{|c|}{$6.70 \pm 0.66$} \\
\hline SVF & \multicolumn{2}{|c|}{$0.23 \pm 0.05$} & \multicolumn{2}{|c|}{$0.22 \pm 0.04$} \\
\hline
\end{tabular}

\subsubsection{Through-the-surface based model results}

It was found that through-the-surface MCR measurements could accurately predict cartilage sub-

component distributions up to $0.5 \mathrm{~mm}(\approx 25 \%$ of the total thickness in this study) in Chapter 2 . In order

to obtain depth-dependent material property distributions past this depth in through-the-surface

models, a linear regression was fit between the ratio of the recovered stage predicted GAG content to the equivalent cross-section predicted GAG content (Figure 3.4). Three control sample optimizations were then completed using recovered stage through-the-surface GAG measurements scaled with the associated regression line. These models produced optimization values with a percent difference of 4.1 $\pm 12.3 \%$ from their cross-section counterparts. 


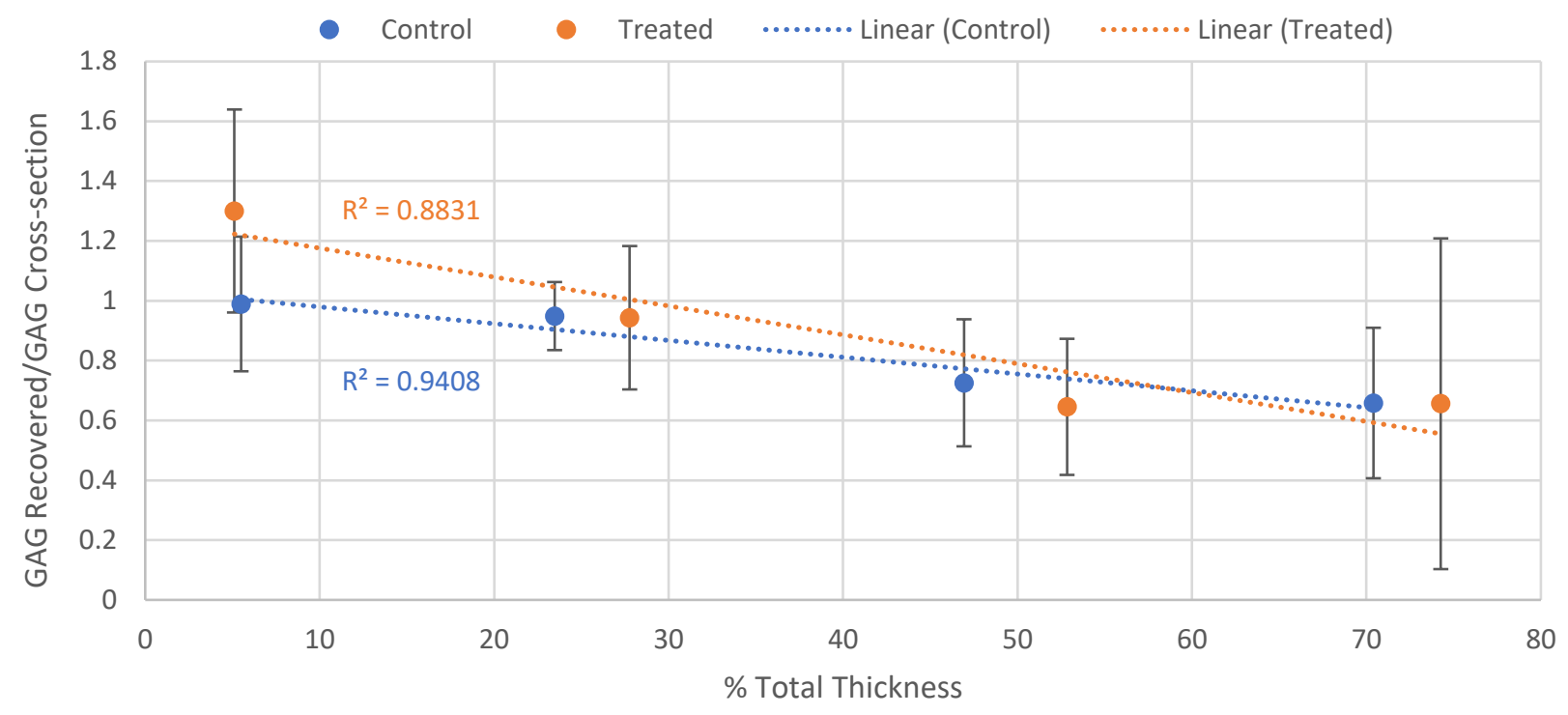

Figure 3.4: Recovered stage through-the-surface MCR prediction of GAG content divided by the equivalent cross-section prediction for treated and control samples alongside regression lines. Error bars represent the $95 \%$ C.I. for the sample distribution. Depth measurements within a $10 \%$ thickness range were averaged into one depth based on negligible change in properties.

\subsubsection{Reaction force predictions}

A typical reaction force plot from simulated and experimental data is shown in Figure 3.5. Model time points were biased towards the beginning of the test to weight the optimization algorithm more heavily in the transient phase. Both the depth-dependent and homogeneous models under-predicted the peak reaction force, reaching $81.9 \pm 3.3 \%$ and $81.5 \pm 5.3 \%$ of the experimental maximum across all samples, respectively. The depth-dependent model consistently reproduced the transition from transient to equilibrium phase more accurately as seen in Figure 3.5(c). The depth-dependent model was also able to reproduce the equilibrium response of the tissue more consistently, reaching $98.4 \pm 7.8 \%$ of the experimental result (averaged over last 10 seconds of test) as opposed to a larger spread of $96.7 \pm 17.4$ $\%$ for the homogeneous model. 

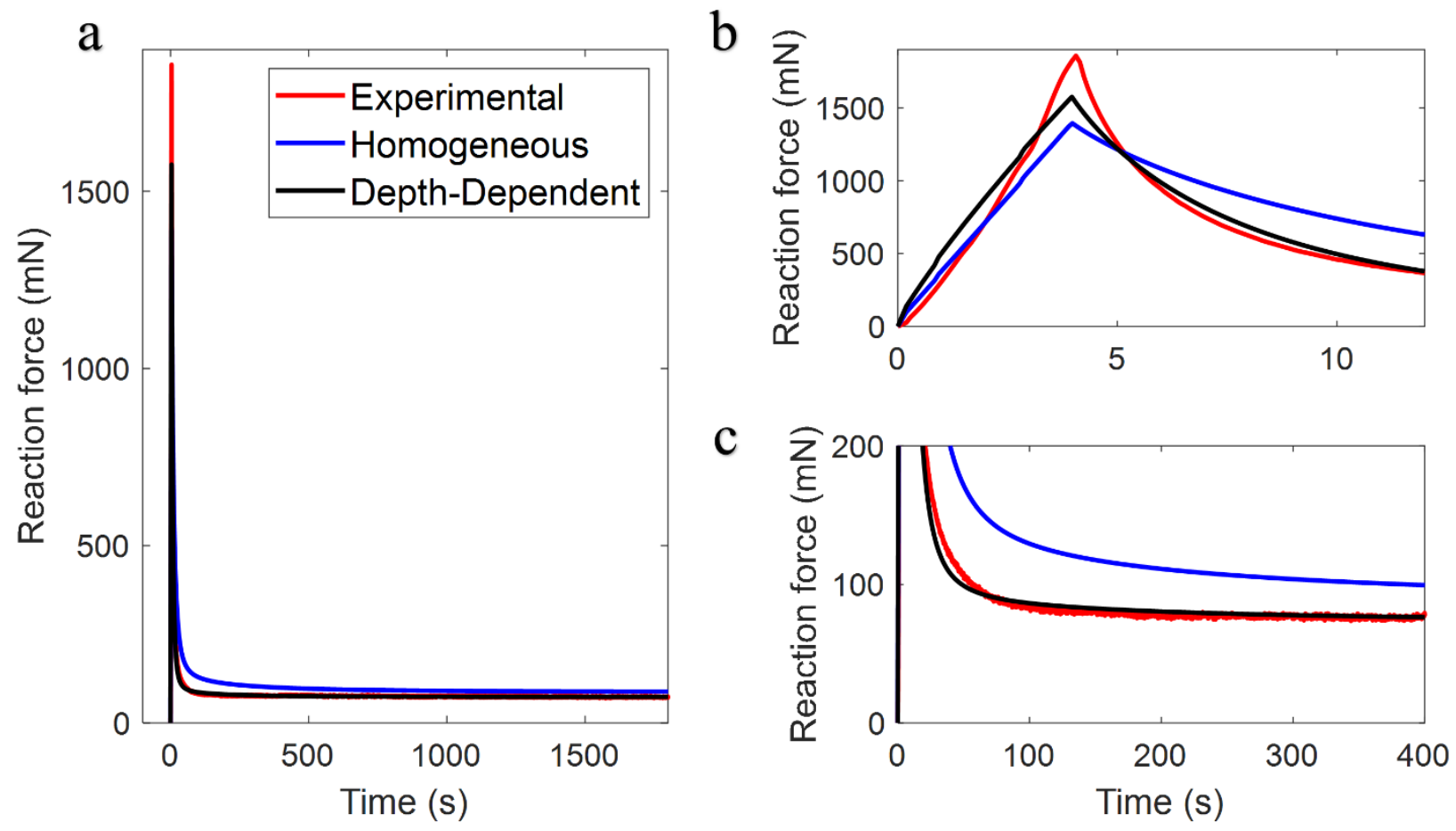

Figure 3.5: (a) Reaction force plot for individual treated sample over the duration of the indentation test. (b) Reaction forces around the end of the ramp displacement showing a comparison of the peak experimental and simulated reaction forces. (c) Comparison of reaction forces near the end of the transient phase of the indentation test.

Peak reaction force percent difference between preclear and recovered loading stages were $6.2 \pm 27.7$ $\%$ and $1.9 \pm 16.4 \%$ for treated and control samples respectively $(p=0.25$ paired t-test within groups between preclear and recovered stages, $p=0.55$ unpaired t-test on percent difference between groups). Similarly, for equilibrium response (average of last $10 \mathrm{~s}$ of test) these percent differences were $0.2 \pm 37.7$ $\%$ and $16.8 \pm 50.2 \%$ for treated and control samples respectively $(p=0.90$ paired t-test within groups between preclear and recovered stages, $p=0.27$ unpaired t-test on percent difference between groups). Based on this result, changes to tissue morphology were associated to time related degradation and not the OCA. This conclusion was also supported by comparison of Raman peak intensity ratios related to collagen II stability (Appendix A). 


\subsubsection{Material response}

Material response metrics at the time of peak reaction force from both the depth-dependent and homogeneous models are shown in Figure 3.6 for a single treated sample. These material responses varied in magnitude across samples, but maintained the same distributions shown below.

Depth-dependent models on average produced peak first principal stresses in the cartilage $680 \%$ larger than their homogeneous counterparts. However, due to the increased transverse $(X-Y)$ fibril stiffness near the surface depth-dependent models only reached $92.2 \pm 9.9 \%$ of the first principal strain values found in homogeneous models. The depth-dependent models had $4.7 \pm 14.1 \%$ higher peak effective fluid pressure than the homogeneous models. While the indenter was descending into the tissue the peak fluid pressure would reach a maximum near the discontinuity at the indenter edge as similarly reported by Warner et al.[87]. This location of maximum fluid pressure migrated towards the center of the indenter as seen in Figure 3.6(c) and the fluid pressure decreased during the stress relaxation period.

Fluid load support, defined here as the ratio of the integral of fluid pressure over the indenter contact area divided by the integral of the contact pressure over the indenter contact area [73], is shown for a single treated sample in Figure 3.7. This response was typical of all samples with the depth-dependent model exceeding the peak percentage of fluid load support of the homogeneous models by $3.6 \pm 6.1 \%$ and declining at a slower rate in comparison. After the first 180 seconds $(10 \%)$ of the test the fluid load support approaches zero, and the material response is mainly governed by the ground substance. 

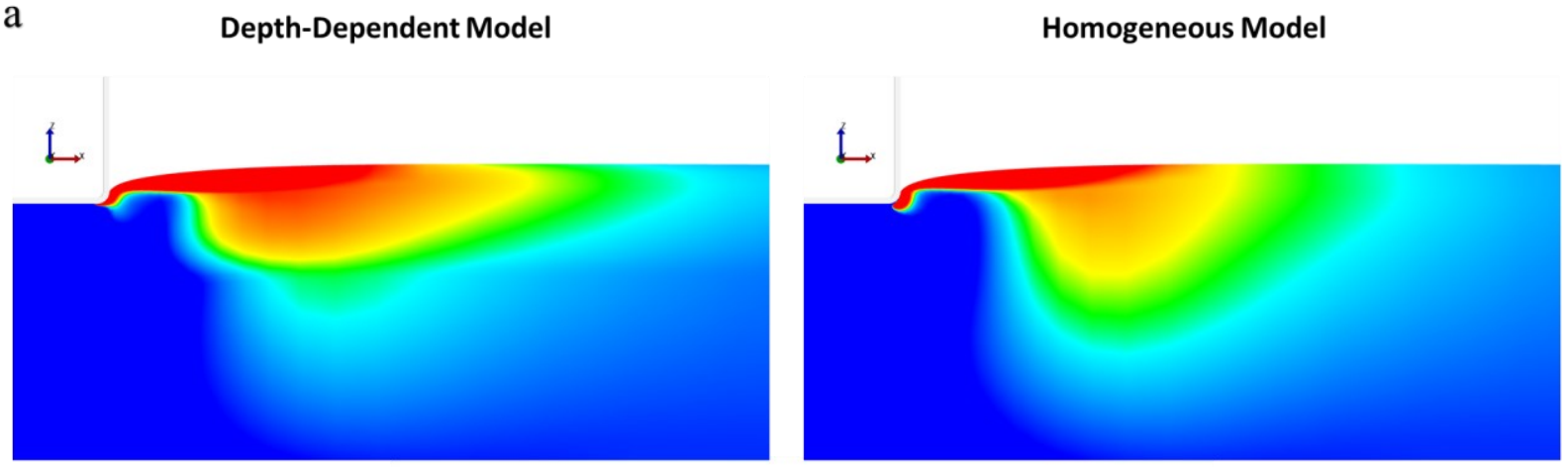

$\begin{array}{lllllllllll}0 & 0.007 & 0.014 & 0.021 & 0.028 & 0.035 & 0.042 & 0.049 & 0.056 & 0.063 & 0.07\end{array}$

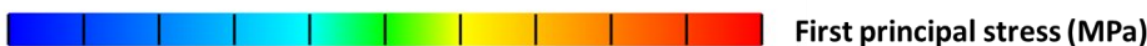

$\mathrm{b}$

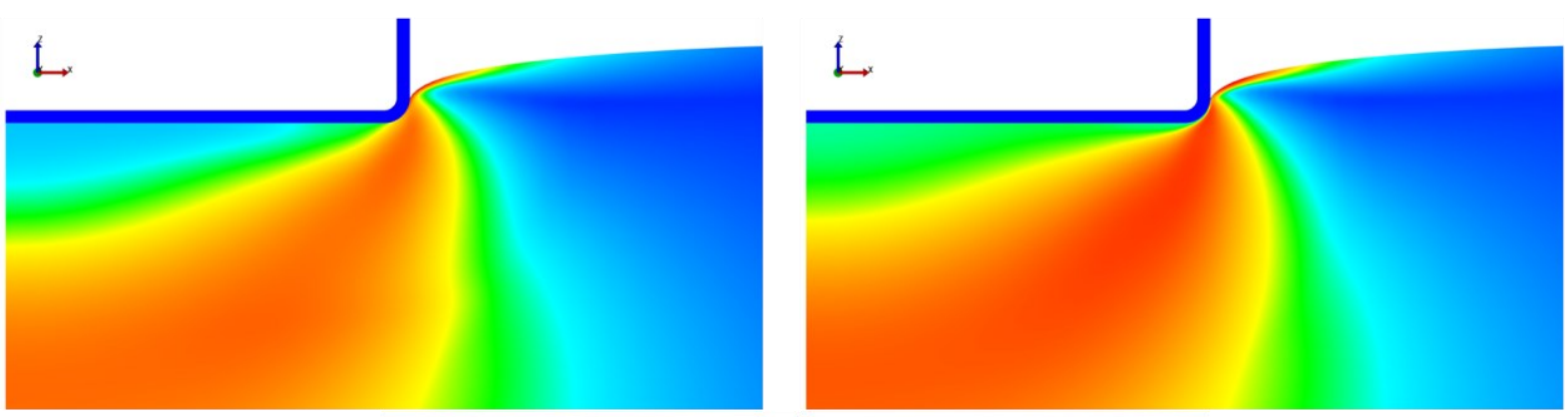

$\begin{array}{lllllllllll}0 & 0.007 & 0.014 & 0.021 & 0.028 & 0.035 & 0.042 & 0.049 & 0.056 & 0.063 & 0.07\end{array}$

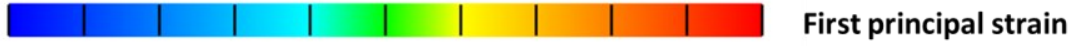

$\mathrm{c}$

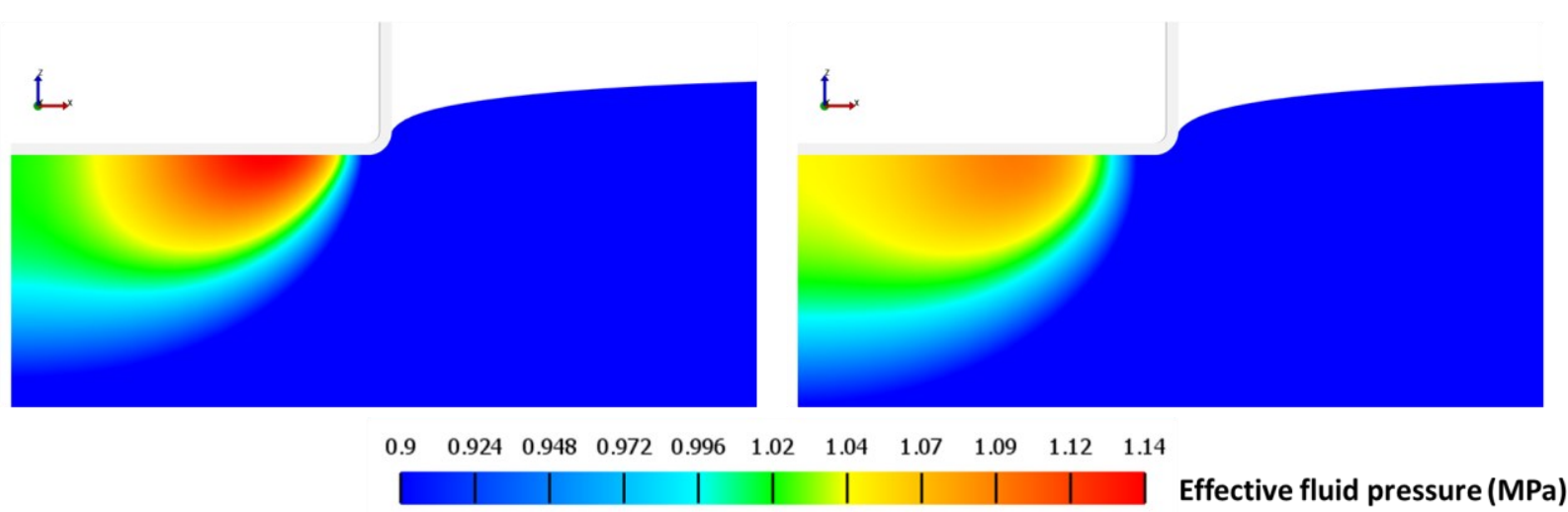

Figure 3.6: (a) First Principal stress (MPa) at peak reaction force time for the depth-dependent and homogeneous models in a treated sample. Higher stresses were concentrated near the surface in the depth-dependent model compared to the homogeneous model. (b) First Principal strain at peak reaction force time for the depth-dependent and homogeneous models in a treated sample. (c) Effective fluid pressure at peak reaction force time for the depth-dependent and homogeneous models in a treated sample. Higher fluid pressures were observed in the depth-dependent model compared to the homogeneous model. 


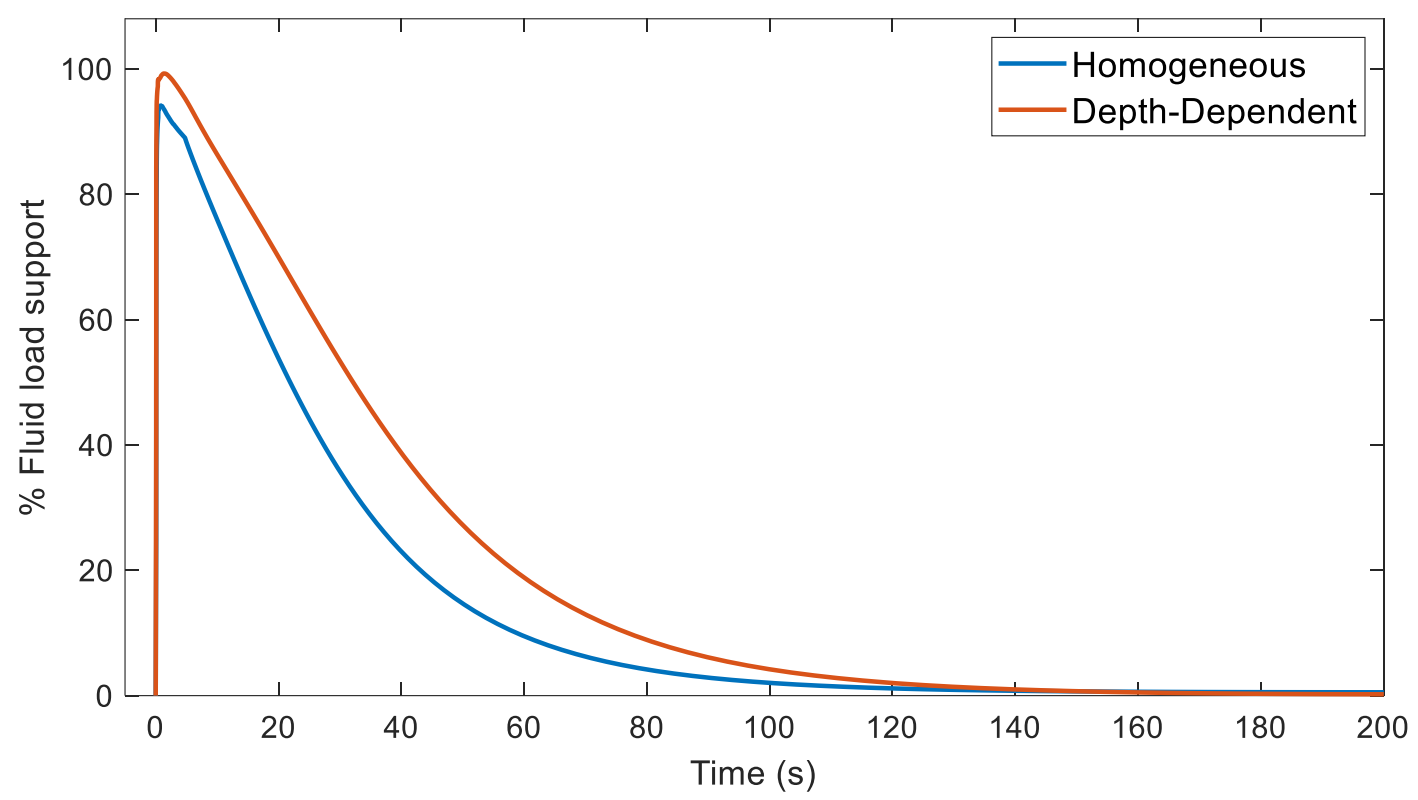

Figure 3.7: Percent fluid load support of the stress-relaxation test for a single treated sample.

\subsection{Discussion}

This study showed that depth and composition-based models of cartilage can reproduce experimental behavior. Optimized depth-dependent models produced smaller errors in reaction force, and concentrated fluid pressure near the surface compared to homogeneous models. This is essential for maintaining fluid load support $[56,58-60]$. Thus, Raman spectroscopy is a useful tool for determining depth-dependent material property distributions in articular cartilage. Depth-dependent models showed an average reduction in the percent difference of optimization function values of approximately $18 \%$ over homogeneous models. Moreover, when using recovered stage through-the-surface MCR measurements with a linearly regressed scaling line, results closely matched the cross-section models. This shows non-destructive Raman measurements made through-the-surface, similar to reported uses in arthroscopic surgeries $[32,33]$ could be of use for FE modeling. This would have direct applications in improving modeling of OA as it develops, however the linearly scaling GAG relationship must be 
evaluated in degenerated cartilage samples. Furthermore, depth-dependent material properties based on composition could be used to study chondrocyte mechanobiology, where an accurate assessment of the cell micromechanical environment could explain depth-dependent cellular activity [88].The OCA used in this study did not improve the depth of resolution of the Raman spectroscopy-based MCR deconvolutions through-the-surface. The OCA used also does not appear to affect tissue morphology based on a recovery of mechanical response in treated samples.

Both depth-dependent and homogeneous models failed to predict the peak reaction force in all samples as seen in Figure 3.5. This is likely due to the simplifications made with the biphasic material used in modeling. Firstly, a Poisson's ratio and hydraulic permeability dependent on strain were not accounted for; both of which are observed phenomenon in articular cartilage $[64,67]$. The model also did not incorporate an ionic-phase, accounting for the Donnan osmotic swelling pressure in the tissue which contributes to compressive stiffness $[62,63]$. The lack of intrinsic flow-independent viscoelasticity in the solid matrix likely accounts for a large portion of the error in the transient phase, especially around the peak. This viscoelasticity has been reported as an important component in predicting the response of cartilage in tension and compression [65]. Another result of neglecting this material property is that its effects are attributed to the elastic properties of the tissue. As a consequence, the fiber tensile modulus parameters found may be overpredicted as noted by Chen et al. [62]. It is also important to note that the largest tissue deformations in indentation testing are in the superficial zone. Due to this, the tissue response is dominated by the material properties in this top layer, potentially neglecting contributions from the other zones [62].

In spite of these inaccuracies the optimized material parameters in both models are comparable to other studies using similar constitutive relationships and indentation tests. Ruggiero et al. [89] reported a closely matching superficial and middle zone compressive modulus of $0.028 \mathrm{MPa}$ and $0.14 \mathrm{MPa}$ respectively in porcine jaw cartilage. Homogeneous models also predicted compressive and fiber tensile 
moduli the same order of magnitude as those reported by Chen et al.[62], and Speirs and Shegaf [69]. However, these studies predicted higher values likely due to use of stiffer bovine knee cartilage. All of these studies reported permeability on a similar order of magnitude $\left(10^{-15} \cdot \mathrm{m}^{4} / \mathrm{N} \cdot \mathrm{s}\right)$. A notable feature of each reported material parameter is the high inter-specimen variability, similarly found in this study and highlighted by the distributions in Table 3.1. This emphasizes the need for sample specific properties to accurately reproduce tissue response, accommodated by Raman spectroscopy. Use of generic parameter distributions may lead to misinterpretations between observed compositional changes in tissue and predicted material responses. Also based on this point, the practicality of using the averaged scaling line for through-the-surface Raman measurements would be dependent on using cross-section standards which are closely related.

In contrast to the transient phase both the homogeneous and depth-dependent models predicted reaction forces in the equilibrium phase closely matching the experimental result. However, the depthdependent model predicted a response that was more consistent with previous studies. As reported in these previous studies using depth-dependent cartilage models, an increase in tensile stress near the articular surface was observed in conjunction with an increase in fluid pressure $[56,58,59,68]$. As previously noted $[56,60]$ this increased fluid pressure in the depth-dependent model is related to the disparity between the fiber tensile modulus and ground substance compressive modulus in the superficial zone. Coupling this relationship with the low Poisson's ratio, elements do not substantially expand in the transverse direction $(X-Y)$ as they compress in the axial direction ( $Z$ ) from loading. As a result, more fluid must be forced out of the pores, for an equivalent axial compression, increasing fluid pressure. Figure 3.6(a) shows how another by-product of this tensile and compressive modulus disparity is a larger recruitment of the superficial zone to support the indenter strain in the depth-dependent model. Because of this, lower layers are shielded from tensile stress in comparison to the homogeneous model. This difference in predicted stress demonstrates the importance of linking sample specific 
composition changes that would be observed in $\mathrm{OA}$, to tissue response. This has the potential to change our understanding of how physiological loading affects, and is affected by, OA development. The stated modulus relationship also reduces the maximum tensile strain directly below the center of the indenter for depth-dependent models as seen in Figure 3.6(b). While the increase in fluid pressure was relatively small for the single ramp displacement ( $4 \%$ increase in depth-dependent models) the effects of the tensile-compressive modulus disparity would be more apparent in cyclic loading. Evidence for this is also shown here by the increased and sustained fluid load support ratio seen in Figure 3.7. This difference in the transient phase would likely be increased under cyclic loading which occurs with much shorter cycle times in-vivo $(\approx 1 \mathrm{~s})$ compared to the relaxation time of this experiment. In this loading configuration the disparity in moduli is key for maintaining fluid pressurization as shown by Mäkelä and Korhonen [90]. This is an important aspect of articular cartilage loading which decreases friction and improves wear resistance in the tissue $[56,58-60]$.

Past studies have shown that the use of polarized light microscopy or magnetic resonance imaging for determining collagen fibril orientation can improve FE modeling of cartilage $[56,57,91]$. As a simplified fiber orientation distribution was used in depth-dependent models for this study, use of polarized Raman spectroscopy for determining fibril orientations could further improve models [20]. The theoretically predicted tensile stiffness of the fibrils could also be validated by use of tensile testing [92]. Preliminary results using depth-dependent permeability were shown to have little effect on improving optimization values. However, inaccuracies associated with using Raman-based MCR in the 400-1800 $\mathrm{cm}^{-1}$ range may have contributed to this result (Chapter 2). Future work increasing the number of data points along the tissue thickness, along with using MCR across the $0-3200 \mathrm{~cm}^{-1}$ range, may improve these results. In addition, incorporating permeability with strain dependence and flow-independent ground substance viscoelasticity will likely improve the transient response of the material. Further work using alternative OCAs and clearing techniques could also enhance through-the-surface measurements 
for modeling purposes. A key assumption which must be confirmed in future studies is whether the compressive stiffness of the ground substance can be proportionally scaled with GAG content. Bergholt et al. [20] reported this correlation could not be found when using nano-indentation testing throughout the depth of cartilage cross-sections. However, this conclusion is yet to be exhaustively tested.

\subsection{Conclusion}

Application of Raman spectroscopy-based MCR measurements of depth-dependent sub-component distributions in cartilage were shown to improve tissue response in FE models. Depth-dependent models using Raman measurements made through-the-surface, with scaling factors, also proved capable of predicting material responses similar to their cross-section counterparts. This shows Raman spectroscopy-based MCR measurements made in-situ or in-vivo could potentially be useful in nondestructively assessing degenerative changes in cartilage over time. This would have direct applications in modeling of joint diseases such as osteoarthritis as they develop. This would also be applicable in examining the evolution of properties as the matrix is recapitulated in engineered tissue. While the OCA used in this study did not improve the resolution of Raman measurements through-the-surface, alternate OCAs and clearing techniques could prove more beneficial. 
Chapter 4

Discussion and Conclusion 


\section{Discussion and Conclusion}

Raman spectroscopy-based MCR measurements of cartilage through-the-surface can reliably predict sub-component distributions up to $0.5 \mathrm{~mm}$ - representing $25 \%$ of the cartilage thickness in this study. This study also showed that depth and composition-based FE models of cartilage created using Raman spectroscopic measurements can reproduce experimental behavior. Optimized depth-dependent models using Raman data from cross-sections reduced error in predicted reaction force by approximately $18 \%$ compared to homogeneous models. They also concentrated fluid pressure near the surface which is essential for maintaining fluid load support. Thus, Raman spectroscopy is a useful tool for determining depth-dependent material property distributions in articular cartilage. Through-thesurface Raman measurement-based models also closely matched their cross-section equivalents. This shows that Raman measurements made in-situ or in-vivo could be applicable for FE modeling of cartilage.

The clearing solution used in this study does not appear to have any benefit in regards to increasing the depth of resolution for through-the-surface Raman measurements. Despite the potential for urea to denature the proteins in cartilage, it was determined that the OCA did not affect tissue morphology. This was concluded based on a recovery in tissue mechanical response noted in Section 3.4.3 between the preclear and recovered loading stages. Furthermore, changes in Raman band intensity ratios between these stages related to collagen stability (mentioned in Section 1.6) were assessed in Appendix A. Only a small positive difference, indicating dehydration [42], is present in some samples for the $938 / 922 \mathrm{~cm}^{-1}$ ratio. This was deemed related to time dependent degradation as it is shared among both treated and control samples. Further details on the scripts used to process Raman data are shown in Appendix E. 


\subsection{Limitations}

Several limitations were found regarding the testing methods used in this thesis. MCR deconvolution in the $400-1800 \mathrm{~cm}^{-1}$ range led to unrealistic $(>1.0 \mathrm{~g} / \mathrm{mL})$ water concentrations near the surface of cartilage. This was due to the low molecular specificity of the water spectra in the measurement range [21]. As previously reported MCR deconvolutions should ideally include the $\mathrm{O}-\mathrm{H}$ stretching vibration in a $3200-3650 \mathrm{~cm}^{-1}$ range to improve accuracy [21]. Lack of an appropriate grating in the spectrometer used, prevented this range expansion to reach the high-wavenumber Raman shifts. This error in predicted water distributions also likely impacted the preliminary FE models with depth-dependent permeability. The methods and results used to compare these depth-dependent permeability models with those in Chapter 3 are shown in Appendix D. Based on a negligible change in optimization function value (sum of squared difference) of these depth-dependent permeability models from their constant permeability counterparts this property was neglected. This is a notable potential topic for future research.

Referencing depth measurements based on surface boundaries proved challenging for cartilage. Due to the steep composition gradients near the surface boundary in cartilage, care must be taken in specifying these reference heights. Some Raman based studies of connective tissue have found success using area under the curve measurements of certain Raman peaks for this purpose $[42,93]$. However, use of a wire with known reference dimensions led to more timely zeroing measurements with acceptable tolerances ( $\pm 29 \mu \mathrm{m}$ described in Section 2.5). For application in-situ or in-vivo different reference height measurement methods should be explored which strike a balance between accuracy and speed of acquisition.

While depth-dependent FE models did show an improved tissue response over homogeneous models, inaccuracies still exist due to simplifying assumptions. This error was particularly noticeable in the 
transient phase of loading. Some of these inaccuracies include the lack of flow-independent viscoelasticity in the ground substance, lack of an ionic phase, and permeability and a Poisson's ratio independent of strain. The loading regime for modeling tissue response was also limited to uniaxial indentation testing. The response from this type of loading is more heavily influenced by the material properties of the superficial zone [62]. As a result, contributions from deeper zones are potentially neglected affecting the accuracy of the predicted material parameters. Supplemental information for each FE model is provided in Appendix C.

Similarly found across all measurements in this thesis, and others involving cartilage $[20,21,62,81,89]$, is the high amount of inter-specimen variability. Due to this fact it is difficult to assign generic material property distributions across any specimen. This emphasizes the need for sample specific cartilage measurements, which can be accommodated by Raman spectroscopy. This also means that application of regressed lines to adjust Raman data at depth for measurements in-situ or in-vivo would be dependent on the use of closely related cross-section standards. The large variability of the material parameter scaling terms in Table 3.1 suggests that properties may be poorly estimated from just Raman spectra and MCR analysis, and may require mechanical testing in each case. However, use of spectra that include high wavenumbers can better estimate water content [21], and may improve the composition estimates and reduce the scaling factor variability. Intra-specimen variability was low in comparison as highlighted by the consistency in through-the-surface MCR scores shown in Figure 2.5, and reaction force responses in Table C.3 and C.4. While the sample size used was deemed adequate for the purposes of this proof of concept study, future works would benefit from better describing the population distribution with larger sample sizes.

Multiple assumptions and measurements made within this thesis provide compounding error. Position error in the needle and indentation test linear actuator was $\pm 1.27 \mu \mathrm{m}(0.9 \%$ of average total indenter displacement) for a full stroke, and $\pm 0.1 \%$ of full-scale combined error in loadcell reaction forces. 
Caliper measurement error associated with volume measurements used in Section 2.3.7 was difficult to quantify due the tissue softness. However, the initial thickness measurements were carried out in a similar manner and were calculated to have an average difference of $3 \%$ from needle thickness tests. Five samples relied on the $75 \%$ thickness spectra replacing the $100 \%$ measurement, due to the latter containing large bone contributions in its spectra. However, this was deemed a valid approximation due to the relatively constant composition found between the two measurement locations in the remaining seven samples. Error associated with MCR results are not quantifiable in this thesis due to a lack of a comparison standard, but likely contributed the majority of measurement error. Albro et al.[21] has reported correlation coefficients of 0.88 and 0.83 for GAG and collagen respectively in similar measurements for MCR predicted distributions vs. assay tests. The remaining unavoidable systematic error would be associated with using defined geometries (modeling the specimen as perfect cylinder) and boundaries (strict tissue-bone interface) for biological tissues.

\subsection{Future work}

Numerous possibilities exist for future research based on the results from this thesis. Quantifying the ability of Raman-based MCR to identify compositional changes in heathy vs. OA tissue must be conducted to determine its applicability in-situ or in-vivo. These studies would have to assess the sensitivity of MCR-ALS in detecting changes to cartilage spectra based on known stages of tissue degeneration. However, this holds promise as $\mathrm{OA}$ is characterized by progressive changes in composition starting from the surface [4], where this measurement technique was most reliable. This will also likely involve the use of machine learning algorithms to assist in defining classification thresholds. In addition to this point, the results obtained in this thesis mainly constitute training data for fitting Raman measurements to compositional distributions of cartilage. Future studies should show the 
application of data based on test sets, validating model predictions as has been done by Albro et al.[21] with assay comparisons. Further work to this would be based on assessing data obtained arthroscopically using spectrometers. The feasibility of using spectrometers for this purpose has recently been proven using near-infrared and Raman spectroscopes [31-33]. Successful distinction of healthy and damaged tissue in this scenario would offer an import means of assessing OA as it develops. These tests could also offer an in-situ means of assessing engineered cartilage through stages of development [94]. Typical joint surface curvature in-vivo would likely not affect measurements due to small probe sizes ranging from 2-3 mm in diameter [32, 33]. However, as noted by Prakash et al. [32] a limiting factor is narrow joint spaces resulting in suboptimal probe alignment.

Many past studies have used polarized Raman spectroscopy for determining collagen fibril orientations in tissue $[20,43,95,96]$. While the change in intensity of specific collagen peaks due to orientation is relatively easy to quantify in cross-sections, the attenuation linked to in-situ or in-vivo measurements would make this task more challenging. Studies quantifying to what depth collagen orientation can be accurately determined are another potential route for future studies. One possibility for this would be using MCR in conjunction with reference Raman spectra of preferentially arranged collagen fibrils. This may offer a means of quantifying orientation ratios in all directions for a discrete volume or layer of tissue, as opposed to an averaged value [20]. Applying this information to FE models of cartilage may also improve sample specific predictions in tissue response, as has similarly been done using MRI [56]. Using static uniaxial indentation is not the most accurate means of quantifying the entire depthdependent mechanical properties of soft tissues like cartilage $[62,97]$. Other common means of testing cartilage like confined/unconfined compression also have drawbacks due to the non-physiological stresses they can impose [69], and require careful specimen preparation. As a result, studies employing biaxial dynamic loading of cartilage have been shown to better simulate typical physiological loading of the tissue [69]. This in turn could offer better insight into how the depth-dependent sub-components of 
cartilage interact and respond to loading in-vivo. Future studies using depth-dependent distributions from Raman spectroscopy in combination with biaxial dynamic loading would offer improved predictions of tissue response. These future studies should also try to model the flow-independent viscoelasticity of the tissue through multiple independent stress relaxation tests under both tension and compression. However, differentiating flow dependent and independent viscoelastic effects is difficult with individual curve fitting optimizations due to the large number of parameters involved resulting in overfitting. Typically these tissue behaviours require direct measurement of the hydraulic permeability or dynamic shear loading $[65,98]$.

Alternate clearing solutions, or longer exposure to higher concentrations of FRUIT, may improve through-the-surface measurements in future studies. The important aspect of choosing these alternative OCAs will revolve around reducing spectral overlap with important collagen features as mentioned in Chapter 2. Preliminary tests using the formamide based Clear ${ }^{\mathrm{T2}}$ [99] OCA offered a promising alternative to the fructose-based FRUIT [74] due its speed in clearing (1.5 $\mathrm{h}$ vs. $12 \mathrm{~h}$ ). This solution also did not appear to affect tissue morphology based on a recovery in tissue response between preclear and recovered stages. Numerous other options also exist for optical clearing which could improve results $[49,50]$.

\subsection{Conclusion}

This thesis demonstrated the feasibility of using Raman spectroscopy in conjunction with MCR to determine the distribution of cartilage sub-components through-the-surface. Raman measurements made through-the-surface could reliably predict these distributions up to a depth of approximately 0.5 $\mathrm{mm}$. Optical clearing with the FRUIT based OCA did not improve these measurements. Furthermore, it 
was determined a potential OCA must homogenize tissue scattering enough to overcome the inherent error associated with additions to the total number of sub-components MCR-ALS solves for.

Depth-dependent FE models using material property distributions based on Raman data improved predictions of tissue response in comparison to simplified homogeneous models. Error in predicted reaction force from these depth-dependent models was reduced by $18 \%$ in comparison to homogeneous models. Through-the-surface Raman measurement-based FE models also performed similarly to their cross-section counterparts. This demonstrates how Raman spectroscopic measurements made in-situ or in-vivo could improve current modeling of cartilage. Applications of this measurement technique arthroscopically could yield an improved understanding of how compositional changes observed in early stage OA affect tissue response. This may provide better insight into potential treatments.

\subsection{Contributions to field}

This thesis showed that Raman spectroscopy-based MCR measurements of cartilage sub-components can improve FE modeling of the tissue. The tissue response predicted by these depth-dependent models was also analysed and shown to be superior to commonly used homogeneous models $[62,69]$. The depth to which Raman spectroscopy-based MCR measurements could accurately discern this composition was also quantified. It was demonstrated that this measurement technique could be of use in-situ or in-vivo based on results obtained through-the-surface. 


\section{References}

[1] V. C. Mow, A. Ratcliffe, and A. Robin Poole, "Cartilage and diarthrodial joints as paradigms for hierarchical materials and structures," Biomaterials, vol. 13, no. 2, pp. 67-97, 1992, doi: 10.1016/0142-9612(92)90001-5.

[2] A. J. S. Fox, A. Bedi, and S. Rodeo, "The Basic Science of articular cartilage: structure, composition, and Function," Sports Health, vol. 1, no. 6, pp. 461-468, 2009.

[3] R. Marks and J. P. Allegrante, "Prevalence and impact of arthritis: opportunities for prevention," Health Educ. J., vol. 66, no. 1, pp. 3-21, 2007, doi: 10.1177/0017896907069366.

[4] H. Muir, "Cartilage structure and metabolism and basic changes in degenerative joint disease," Aust. N. Z. J. Med., vol. 8, pp. 1-5, 1978, doi: 10.1111/j.1445-5994.1978.tb04770.x.

[5] C. Bombardier, G. Hawker, and D. Mosher, "The impact of arthritis in Canada: today and over the next 30 years," 2011. [Online]. Available: http://www.arthritisalliance.ca.

[6] J. W. P. Michael, K. U. Schlüter-Brust, and P. Eysel, "The epidemiology, etiology, and treatment of osteoarthritis of the knee," Dtsch. Arztebl., vol. 107, no. 9, pp. 152-162, 2010, doi:

10.3238/arztebl.2010.0152.

[7] D. T. Felson, "Epidemiology of hip and knee osteoartrritis," Epidemiol. Rev., vol. 10, no. 1, pp. 128, 1988, doi: 10.1093/oxfordjournals.epirev.a036019.

[8] MacDonald KV, Sanmartin C, Langlois K, Marshall DA. "Symptom onset, diagnosis and management of osteoarthritis," Health Rep. 2014 Sep;25(9):10-7. PMID: 25229896.

[9] R. F. Loeser, S. R. Goldring, C. R. Scanzello, and M. B. Goldring, "Osteoarthritis: A disease of the joint as an organ," Arthritis Rheumatol., vol. 64, no. 6, pp. 1697-1707, 2012, doi: 10.1038/jid.2014.371.

[10] C. R. Ethier and C. A. Simmons, Introductory biomechanics: from cells to organisms, 1st ed. New York: Cambridge University Press, 2007.

[11] M. F. Barbe, J. Driban, A. E. Barr, S. N. Popoff, and F. F. Safadi, Bone Pathology, 2nd ed. Philadelphia, PA: Humana Press, 2009.

[12] C. P. Neu, K. Komvopoulos, and A. H. Reddi, "The interface of functional biotribology and regenerative medicine in synovial joints," Tissue Eng. - Part B Rev., vol. 14, no. 3, pp. 235-247, 2008, doi: 10.1089/ten.teb.2008.0047.

[13] J. J. Liao, S. Miramini, X. Liu, and L. Zhang, "Computational study on synovial fluid flow behaviour in cartilage contact gap under osteoarthritic condition," Comput. Biol. Med., vol. 123, 2020, doi: 10.1016/j.compbiomed.2020.103915.

[14] A. P. Newman, "Articular cartilage repair," Am. J. Sports Med., vol. 26, no. 2, pp. 309-324, 1998, doi: 10.1016/j.jajs.2014.06.003.

[15] V. C. Mow, S. C. Kuei, W. M. Lai, and C. G. Armstrong, "Biphasic creep and stress relaxation of articular cartilage in compression: Theory and experiments," J. Biomech. Eng., vol. 102, no. 1, pp. 73-84, 1980, doi: 10.1115/1.3138202. 
[16] R. Ellis, E. Green, and C. P. Winlove, "Structural analysis of glycosaminoglycans and proteoglycans by means of Raman microspectrometry," Connect. Tissue Res., vol. 50, no. 1, pp. 29-36, 2009, doi: $10.1080 / 03008200802398422$.

[17] V. Klika, E. A. Gaffney, Y. C. Chen, and C. P. Brown, "An overview of multiphase cartilage mechanical modelling and its role in understanding function and pathology," J. Mech. Behav. Biomed. Mater., vol. 62, pp. 139-157, 2016, doi: 10.1016/j.jmbbm.2016.04.032.

[18] G. Stell and C. G. Joslin, "The Donnan equilibrium: A theoretical study of the effects of interionic forces," Biophys. J., vol. 50, no. 5, pp. 855-859, 1986, doi: 10.1016/S0006-3495(86)83526-3.

[19] S. Akizuki, V. C. Mow, F. Müller, J. C. Pita, D. S. Howell, and D. H. Manicourt, "Tensile properties of human knee joint cartilage: I. Influence of ionic conditions, weight bearing, and fibrillation on the tensile modulus," J. Orthop. Res., vol. 4, no. 4, pp. 379-392, 1986, doi: $10.1002 /$ jor.1100040401.

[20] M. S. Bergholt, J. P. St-Pierre, G. S. Offeddu, P. A. Parmar, M. B. Albro, J. L. Puetzer, et al., "Raman spectroscopy reveals new insights into the zonal organization of native and tissue-engineered articular cartilage," ACS Cent. Sci., vol. 2, no. 12, pp. 885-895, 2016, doi: 10.1021/acscentsci.6b00222.

[21] M. B. Albro, M. S. Bergholt, J. P. St-Pierre, A. V Guitart, H. M. Zlotnick, E. G. Evita, et al., "Raman spectroscopic imaging for quantification of depth-dependent and local heterogeneities in native and engineered cartilage," npj Regen. Med., vol. 3, no. 1, pp. 1-11, 2018, doi: 10.1038/s41536018-0042-7.

[22] E. S. Oswald, P. G. H. Chao, J. C. Bulinski, G. A. Ateshian, and C. T. Hung, "Dependence of zonal chondrocyte water transport properties on osmotic environment," Cell. Mol. Bioeng., vol. 1, no. 4, pp. 339-348, 2008, doi: 10.1007/s12195-008-0026-6.

[23] E. M. Darling, R. E. Wilusz, M. P. Bolognesi, S. Zauscher, and F. Guilak, "Spatial mapping of the biomechanical properties of the pericellular matrix of articular cartilage measured in situ via atomic force microscopy," Biophys. J., vol. 98, no. 12, pp. 2848-2856, 2010, doi: 10.1016/j.bpj.2010.03.037.

[24] R. Kumar, K. M. Grønhaug, N. K. Afseth, V. Isaksen, C. de Lange Davies, J. O. Drogset, et al., "Optical investigation of osteoarthritic human cartilage (ICRS grade) by confocal Raman spectroscopy: a pilot study," Anal. Bioanal. Chem., vol. 407, no. 26, pp. 8067-8077, 2015, doi: 10.1007/s00216-015-8979-5.

[25] S. R. Goldring and M. B. Goldring, "The role of cytokines in cartilage matrix degeneration in osteoarthritis," Clin. Orthop. Relat. Res., no. 427S, pp. S27-S26, 2004, doi: 10.1097/01.blo.0000144854.66565.8f.

[26] L. A. Setton, V. C. Mow, F. J. Müller, J. C. Pita, and D. S. Howell, “Mechanical properties of canine articular cartilage are significantly altered following transection of the anterior cruciate Ligament," J. Orthop. Res., vol. 12, no. 4, pp. 451-463, 1994, doi: 10.1002/jor.1100120402.

[27] J. T. A. Mäkelä, S. K. Han, W. Herzog, and R. K. Korhonen, "Very early osteoarthritis changes sensitively fluid flow properties of articular cartilage," J. Biomech., vol. 48, no. 12, pp. 3369-3376, 2015, doi: 10.1016/j.jbiomech.2015.06.010. 
[28] M. E. Mononen, P. Tanska, H. Isaksson, and R. K. Korhonen, "New algorithm for simulation of proteoglycan loss and collagen degeneration in the knee joint: Data from the osteoarthritis initiative," J. Orthop. Res., vol. 36, no. 6, pp. 1673-1683, 2018, doi: 10.1002/jor.23811.

[29] S. Mukherjee, M. Nazemi, I. Jonkers, and L. Geris, "Use of computational modeling to study joint degeneration: a review," Front. Bioeng. Biotechnol., vol. 8, no. 93, pp. 1-20, 2020, doi: 10.3389/fbioe.2020.00093.

[30] J. H. Kellgren and J. S. Lawrence, "Radiological assessment of osteo-arthritis," Ann. Rheum. Dis., vol. 16, no. 4, pp. 494-502, 1957.

[31] J. K. Sarin, O. Nykänen, V. Tiitu, I. A. D. Mancini, H. Brommer, J. Visser, et al., "Arthroscopic determination of cartilage proteoglycan content and collagen network structure with nearinfrared spectroscopy," Ann. Biomed. Eng., vol. 47, no. 8, pp. 1815-1826, 2019, doi: 10.1007/s10439-019-02280-7.

[32] M. Prakash, A. Joukainen, J. Torniainen, M. K. M. Honkanen, L. Rieppo, I. O. Afara, et al., "Nearinfrared spectroscopy enables quantitative evaluation of human cartilage biomechanical properties during arthroscopy," Osteoarthr. Cartil., vol. 27, no. 8, pp. 1235-1243, 2019, doi: 10.1016/j.joca.2019.04.008.

[33] K. A. Esmonde-White, F. W. L. Esmonde-White, M. D. Morris, and B. J. Roessler, "Fiber-optic Raman spectroscopy of joint tissues," Analyst, vol. 136, no. 8, pp. 1675-1685, 2011, doi: 10.1039/c0an00824a.

[34] N. S. J. Lim, Z. Hamed, C. H. Yeow, C. Chan, and Z. Huang, "Early detection of biomolecular changes in disrupted porcine cartilage using polarized Raman spectroscopy," J. Biomed. Opt., vol. 16, no. 1, pp. 1-10, 2011, doi: 10.1117/1.3528006.

[35] Y. Takahashi, N. Sugano, M. Takao, T. Sakai, T. Nishii, and G. Pezzotti, "Raman spectroscopy investigation of load-assisted microstructural alterations in human knee cartilage: Preliminary study into diagnostic potential for osteoarthritis," J. Mech. Behav. Biomed. Mater., vol. 31, pp. 77-85, 2014.

[36] J. X. Cheng and X. S. Xie, Coherent Raman scattering microscopy, 1st ed. Boca Raton: CRC Press, 2016.

[37] J. R. Ferraro, K. Nakamoto, and C. W. Brown, Introductory Raman spectroscopy: second edition, 2nd ed. San Diego: Academic Press Inc., 2003.

[38] R. R. Jones, D. C. Hooper, L. Zhang, D. Wolverson, and V. K. Valev, "Raman techniques: fundamentals and frontiers," Nanoscale Res. Lett., vol. 14, no. 231, pp. 1-34, 2019, doi: 10.1186/s11671-019-3039-2.

[39] E. Pavlou, X. Zhang, J. Wang, and N. Kourkoumelis, "Raman spectroscopy for the assessment of osteoarthritis," Ann. Jt., vol. 3, no. 83, pp. 1-10, 2018, doi: 10.21037/aoj.2018.09.10.

[40] T. Nguyen, T. Happillion, J. Feru, S. Brassart-Passco, J. Angiboust, M. Manfait, et al., "Raman comparison of skin dermis of different ages: focus on spectral markers of collagen hydration," J. Raman Spectrosc., vol. 44, no. 9, pp. 1230-1237, 2013, doi: 10.1002/jrs.4355. 
[41] Q. Zhang, K. Chan, G. Zhang, T. Gillece, L. Senak, D. Moore, et al., "Raman microspectroscopic and dynamic vapor sorption characterization of hydration in collagen and dermal tissue,"

Biopolymers, vol. 95, no. 9, pp. 607-615, 2011, doi: 10.1002/bip.21618.

[42] A. Y. Sdobnov, V. Tuchin, J. Lademann, and M. Darvin, "Confocal Raman microscopy supported by optical clearing treatment of the skin - influence on collagen hydration," J. Appl. Phys. D, vol. 50, no. 28, pp. 1-9, 2017, doi: https://doi.org/10.1088/1361-6463/aa77c9.

[43] A. Bonifacio, V. Sergo, and A. Bonifacio, "Effects of sample orientation in Raman microspectroscopy of collagen fibers and their impact on the interpretation of the amide III band," Vib. Spectrosc., vol. 53, no. 2, pp. 314-317, 2010, doi: 10.1016/j.vibspec.2010.04.004.

[44] R. Reddy and R. Bhargava, Chemometric Methods for Biomedical Raman Spectroscopy and Imaging. In: Matousek P., Morris M. (eds) Emerging Raman Applications and Techniques in Biomedical and Pharmaceutical Fields. Berlin: Springer, 2010.

[45] A. de Juan and R. Tauler, "Multivariate Curve Resolution (MCR) from 2000: Progress in concepts and applications," Crit. Rev. Anal. Chem., vol. 36, no. 3-4, pp. 163-176, 2006, doi: 10.1080/10408340600970005.

[46] A. De Juan, J. Jaumot, and R. Tauler, "Multivariate Curve Resolution (MCR). Solving the mixture analysis problem," Anal. Methods, vol. 6, no. 14, pp. 4964-4976, 2014, doi: 10.1039/c4ay00571f.

[47] R. L. McCreery, Raman Spectroscopy for Chemical Analysis, 1st ed., New York: John Wiley \& Sons, Inc., 2000.

[48] J. Jaumot, A. de Juan, and R. Tauler, "MCR-ALS GUI 2.0: New features and applications," Chemom. Intell. Lab. Syst., vol. 140, pp. 1-12, 2015, doi: 10.1016/j.chemolab.2014.10.003.

[49] D. Richardson and J. Lichtman, "Clarifying tissue clearing," Cell, vol. 162, no. 2, pp. 246-257, 2015, doi: 10.1016/j.cell.2015.06.067.

[50] A. Azaripour, T. Lagerweij, C. Scharfbillig, A. E. Jadczak, B. Willershausen, C. J. F. Van Noorden, et al., "A survey of clearing techniques for 3D imaging of tissues with special reference to connective tissue," Prog. Histochem. Cytochem., vol. 53, no. 2, pp. 9-23, 2016, doi: 10.1016/j.proghi.2016.04.001.

[51] E. Hecht, Optics, 5th ed. Essex, England: Pearson Education, 2017.

[52] A. Y. Sdobnov, M. E. Darvin, E. A. Genina, A. N. Bashkatov, J. Lademann, and V. V. Tuchin, "Recent progress in tissue optical clearing for spectroscopic application," Spectrochim. Acta - Part A Mol. Biomol. Spectrosc., vol. 197, pp. 216-229, 2018, doi: 10.1016/j.saa.2018.01.085.

[53] C. Neu, T. Novak, K. Gilliland, P. Marshall, and S. Calve, "Optical clearing in collagen- and proteoglycan-rich osteochondral Tissues," Osteoarthr. Cartil., vol. 23, pp. 405-413, 2015, doi: https://doi.org/10.1016/j.joca.2014.11.021

[54] M. T. Ke, S. Fujimoto, and T. Imai, "SeeDB: a simple and morphology-preserving optical clearing agent for neuronal circuit reconstruction," Nat. Neurosci., vol. 16, no. 8, pp. 1154-1161, 2013, doi: https://doi.org/10.1038/nn.3447. 
[55] A. Bykov, T. Hautala, M. Kinnunen, A. Popov, S. Karhula, S. Saarakkala, et al., "Imaging of subchondral bone by optical coherence tomography upon optical clearing of articular cartilage," J. Biophotonics, vol. 9, no. 3, pp. 270-275, 2016, doi: 10.1002/jbio.201500130.

[56] Q. Meng, S. An, R. A. Damion, Z. Jin, R. Wilcox, J. Fisher, et al., "The effect of collagen fibril orientation on the biphasic mechanics of articular cartilage," J. Mech. Behav. Biomed. Mater., vol. 65, pp. 439-453, 2017, doi: 10.1016/j.jmbbm.2016.09.001.

[57] P. Julkunen, P. Kiviranta, W. Wilson, J. S. Jurvelin, and R. K. Korhonen, "Characterization of articular cartilage by combining microscopic analysis with a fibril-reinforced finite-element model," J. Biomech., vol. 40, no. 8, pp. 1862-1870, 2007, doi: 10.1016/j.jbiomech.2006.07.026.

[58] Y. Dabiri and L. P. Li, "Influences of the depth-dependent material inhomogeneity of articular cartilage on the fluid pressurization in the human knee," Med. Eng. Phys., vol. 35, no. 11, pp. 1591-1598, 2013, doi: 10.1016/j.medengphy.2013.05.005.

[59] R. Krishnan, S. Park, F. Eckstein, and G. A. Ateshian, "Inhomogeneous cartilage properties enhance superficial interstitial fluid support and frictional properties, but do not provide a homogeneous state of stress," J. Biomech. Eng., vol. 125, no. 5, pp. 569-577, 2003.

[60] S. Park, R. Krishnan, S. B. Nicoll, and G. A. Ateshian, "Cartilage interstitial fluid load support in unconfined compression," J. Biomech., vol. 36, no. 12, pp. 1785-1796, 2003, doi: 10.1016/S00219290(03)00231-8.

[61] M. A. Soltz and G. A. Ateshian, "A conewise linear elasticity mixture model for the analysis of tension-compression nonlinearity in articular cartilage," J. Biomech. Eng., vol. 122, no. 6, pp. 576586, 2000, doi: 10.1115/1.1324669.

[62] X. Chen, Y. Zhou, L. Wang, M. H. Santare, L. Q. Wan, X. L. Lu, et al., "Determining tensioncompression nonlinear mechanical properties of articular cartilage from indentation testing," Ann. Biomed. Eng., vol. 44, no. 4, pp. 1148-1158, 2016, doi: 10.1007/s10439-015-1402-8.

[63] G. A. Ateshian, V. Rajan, N. O. Chahine, C. E. Canal, and C. T. Hung, "Modeling the matrix of articular cartilage using a continuous fiber angular distribution predicts many observed phenomena," J. Biomech. Eng., vol. 131, no. 6, pp. 1-34, 2009, doi: 10.1115/1.3118773.

[64] N. O. Chahine, C. C.-B. Wang, C. T. Hung, and G. A. Ateshian, "Anisotropic strain-dependent material properties of bovine articular cartilage in the transitional range from tension to compression," vol. 37, no. 8, 2010, doi: 10.1016/j.jbiomech.2003.12.008.

[65] C. Y. Huang, M. A. Soltz, M. Kopacz, V. C. Mow, and G. A. Ateshian, "Experimental verification of the roles of intrinsic matrix viscoelasticity and tension-compression nonlinearity in the biphasic response of cartilage," J. Biomech. Eng., vol. 125, no. 1, pp. 84-93, 2003, doi: 10.1115/1.1531656.

[66] V. C. Mow, "Biphasic indentation of articular cartilage - II. A numerical algorithm and an experimental study," J. Biomech., vol. 22, no. 8/9, pp. 853-861, 1989, doi: 10.1016/00219290(89)90069-9.

[67] S. Chegini and S. J. Ferguson, "Time and depth dependent Poisson's ratio of cartilage explained by an inhomogeneous orthotropic fiber embedded biphasic model," J. Biomech., vol. 43, no. 9, pp. 1660-1666, 2010, doi: 10.1016/j.jbiomech.2010.03.006. 
[68] K. S. Halonen, M. E. Mononen, J. S. Jurvelin, J. Töyräs, and R. K. Korhonen, "Importance of depthwise distribution of collagen and proteoglycans in articular cartilage-A 3D finite element study of stresses and strains in human knee joint," J. Biomech., vol. 46, no. 6, pp. 1184-1192, 2013, doi: 10.1016/j.jbiomech.2012.12.025.

[69] A. D. Speirs and A. Shegaf, "Articular cartilage recovery response during dynamic biaxial loading," presented at Orthopaedic Research Society: Annual Meeting, AZ, U.S.A., 2020, p. 1509.

[70] Q. Meng, Z. Jin, R. Wilcox, and J. Fisher, "Computational investigation of the time-dependent contact behaviour of the human tibiofemoral joint under body weight," Proc. Inst. Mech. Eng. Part H J. Eng. Med., vol. 228, no. 11, pp. 1193-1207, 2014, doi: 10.1177/0954411914559737.

[71] J. M. Patel, B. C. Wise, E. D. Bonnevie, and R. L. Mauck, "A systematic review and guide to mechanical testing for articular cartilage tissue engineering," Tissue Eng. - Part C Methods, vol. 25, no. 10 , pp. 593-608, 2019, doi: 10.1089/ten.tec.2019.0116.

[72] M. R. DiSilvestro and J. K. F. Suh, "A cross-validation of the biphasic poroviscoelastic model of articular cartilage in unconfined compression, indentation, and confined compression," J. Biomech., vol. 34, no. 4, pp. 519-525, 2001, doi: 10.1016/S0021-9290(00)00224-4.

[73] S. A. Maas, B. J. Ellis, G. A. Ateshian, and J. A. Weiss, "FEBio: Finite elements for biomechanics," J. Biomech. Eng., vol. 134, no. 1, pp. 1-10, 2012, doi: 10.1115/1.4005694.

[74] B. Hou, D. Zhang, S. Zhao, M. Wei, Z. Yang, S. Wang, et al., "Scalable and Dil-compatible optical clearance of the mammalian brain," Front. Neuroanat., vol. 9, no. 19, pp. 1-11, 2015, doi: 10.3389/fnana.2015.00019.

[75] W. H. Simon and D. L. Wohl, "Water content of equine articular cartilage: effects of enzymatic degradation and 'artificial fibrillation,'" Connect. Tissue Res., vol. 9, no. 4, pp. 227-232, 1982, doi: 10.3109/03008208209160266.

[76] B. Hansson, C. H. Allen, S. Qutob, B. Behr, B. Nyiri, V. Chauhan, et al., "Development of a flow cell based Raman spectroscopy technique to overcome photodegeneration in human blood," Biomed. Opt. Express, vol. 10, no. 5, pp. 1-14, 2019, doi: 10.1364/boe.10.002275.

[77] S. J. Choquette, E. S. Etz, W. S. Hurst, D. H. Blackburn, and S. D. Leigh, "Relative intensity correction of Raman spectrometers: NIST SRMs 2241 through 2243 for 785 nm, 532 nm, and 488 nm/514.5 nm excitation," Appl. Spectrosc., vol. 61, no. 2, pp. 117-129, 2007, doi: 10.1366/000370207779947585.

[78] M. Morhac and V. Matousek, "Peak clipping algorithms for background estimation in spectroscopic data," Appl. Spectrosc., vol. 62, no. 1, pp. 91-106, 2008, doi: https://doi.org/10.1366/000370208783412762.

[79] R. Keuleers, H. O. Desseyn, B. Rousseau, C. V Alsenoy, and C. Van Alsenoy, "Vibrational analysis of urea," J. Phys. Chem. A, vol. 103, no. 24, pp. 4621-4630, 1999, doi: 10.1021/jp984180z.

[80] L. Ruggiero, A. Sodo, F. Bruni, and M. A. Ricci, "Hydration of monosaccharides studied by Raman scattering," J. Raman Spectrosc., vol. 49, no. 6, pp. 1066-1075, 2017, doi: 10.1002/jrs.5351.

[81] L. Tong, Z. Hao, C. Wan, and S. Wen, "Detection of depth-depend changes in porcine cartilage after wear test using Raman spectroscopy," J. Biophotonics, vol. 11, pp. 1-8, 2017, doi: 10.1002/jbio.201700217. 
[82] R. K. Nicholas Dias, Yung Peng, "Depth-dependent profiles of glycosaminoglycans in articular cartilage by $\mu \mathrm{MRI}$ and histochemistry," Physiol. Behav., vol. 176, no. 3, pp. 139-148, 2017, doi: 10.1016/j.physbeh.2017.03.040.

[83] D. Mittelstaedt and Y. Xia, "Depth-dependent glycosaminoglycan concentration in articular cartilage by quantitative contrast-enhanced micro-computed tomography," Cartilage, vol. 6, no. 4, pp. 216-225, 2015, doi: 10.1177/1947603515596418.

[84] M. Unal and O. Akkus, "Shortwave-infrared Raman spectroscopic classification of water fractions in articular cartilage ex vivo," J. Biomed. Opt., vol. 23, no. 1, pp. 1-11, 2018, doi:

10.1117/1.jbo.23.1.015008.

[85] R. L. Spilker, J. K. Suh, and V. C. Mow, "A finite element analysis of the indentation stressrelaxation response of linear biphasic articular cartilage," J. Biomech. Eng., vol. 114, no. 2, pp. 191-201, 1992, doi: 10.1115/1.2891371.

[86] G. R. Meloni, M. B. Fisher, B. D. Stoeckl, G. R. Dodge, and R. L. Mauck, "Biphasic finite element modeling reconciles mechanical properties of tissue-engineered cartilage constructs across testing platforms," Tissue Eng. - Part A, vol. 23, no. 13-14, pp. 663-674, 2017, doi: 10.1089/ten.tea.2016.0191.

[87] M. D. Warner, W. R. Taylor, and S. E. Clift, "Finite element biphasic indentation of cartilage: A comparison of experimental indenter and physiological contact geometries," Proc. Inst. Mech. Eng. Part H J. Eng. Med., vol. 215, no. 5, pp. 487-496, 2001, doi: 10.1243/0954411011536082.

[88] M. Wong, P. Wuethrich, M. D. Buschmann, P. Eggli, and E. Hunziker, "Chondrocyte biosynthesis correlates with local tissue strain in statically compressed adult articular cartilage," J. Orthop. Res., vol. 15, no. 2, pp. 189-196, 1997, doi: 10.1002/jor.1100150206.

[89] L. Ruggiero, B. K. Zimmerman, M. Park, L. Han, L. Wang, D. L. Burris, et al., "Roles of the fibrous superficial zone in the mechanical behavior of TMJ condylar cartilage," Ann. Biomed. Eng., vol. 43, no. 11, pp. 2652-2662, 2015, doi: 10.1007/s10439-015-1320-9.

[90] J. T. A. Mäkelä and R. K. Korhonen, "Highly nonlinear stress-relaxation response of articular cartilage in indentation: Importance of collagen nonlinearity," J. Biomech., vol. 49, no. 9, pp. 1734-1741, 2016, doi: 10.1016/j.jbiomech.2016.04.002.

[91] Y. Xia, J. B. Moody, N. Burton-Wurster, and G. Lust, "Quantitative in situ correlation between microscopic MRI and polarized light microscopy studies of articular cartilage," Osteoarthr. Cartil., vol. 9, no. 5, pp. 393-406, 2001, doi: 10.1053/joca.2000.0405.

[92] M. Charlebois, M. D. McKee, and M. D. Buschmann, "Nonlinear tensile properties of bovine articular cartilage and their variation with age and depth," J. Biomech. Eng., vol. 126, no. 2, pp. 129-137, 2004, doi: 10.1115/1.1688771.

[93] S. Mujica Ascencio, C. S. Choe, M. C. Meinke, R. H. Müller, G. V. Maksimov, W. Wigger-Alberti, et al., "Confocal Raman microscopy and multivariate statistical analysis for determination of different penetration abilities of caffeine and propylene glycol applied simultaneously in a mixture on porcine skin ex vivo," Eur. J. Pharm. Biopharm., vol. 104, pp. 51-58, 2016, doi: 10.1016/j.ejpb.2016.04.018. 
[94] N. Bhardwaj, D. Devi, and B. B. Mandal, "Tissue-engineered cartilage: the crossroads of biomaterials, cells and stimulating factors," Macromol. Biosci., vol. 15, no. 2, pp. 153-182, 2015, doi: 10.1002/mabi.201400335.

[95] S. Schrof, P. Varga, L. Galvis, K. Raum, and A. Masic, "3D Raman mapping of the collagen fibril orientation in human osteonal lamellae," J. Struct. Biol., vol. 187, no. 3, pp. 266-275, 2014, doi: 10.1016/j.jsb.2014.07.001.

[96] A. Masic, L. Bertinetti, R. Schuetz, L. Galvis, N. Timofeeva, J. W. C. Dunlop, et al., "Observations of multiscale, stress-induced changes of collagen orientation in tendon by polarized Raman spectroscopy," Biomacromolecules, vol. 12, no. 11, pp. 3989-3996, 2011, doi: $10.1021 / \mathrm{bm} 201008 \mathrm{~b}$.

[97] M. S. Sacks and W. Sun, "Multiaxial mechanical behavior of biological materials," Annu. Rev. Biomed. Eng., vol. 5, pp. 251-284, 2003, doi: 10.1146/annurev.bioeng.5.011303.120714.

[98] W. C. Hayes and A. J. Bodine, "Flow-independent viscoelastic properties of articular cartilage matrix," J. Biomech., vol. 11, no. 8, pp. 407-419, 1978, doi: https://doi.org/10.1016/00219290(78)90075-1.

[99] T. Kuwajima, A. A. Sitko, P. Bhansali, C. Jurgens, W. Guido, and C. Mason, "ClearT: a detergentand solvent-free clearing method for neuronal and non-neuronal tissue," Dev., vol. 140, no. 6, pp. 1364-1368, 2013, doi: 10.1242/dev.091844. 
Appendix A: Raman band intensity ratios 
Description: The differences between specific Raman peak intensities between the preclear and recovered stages are shown for each sample below. Information on these ratios, along with their structural affiliations, are described in Section 1.6.

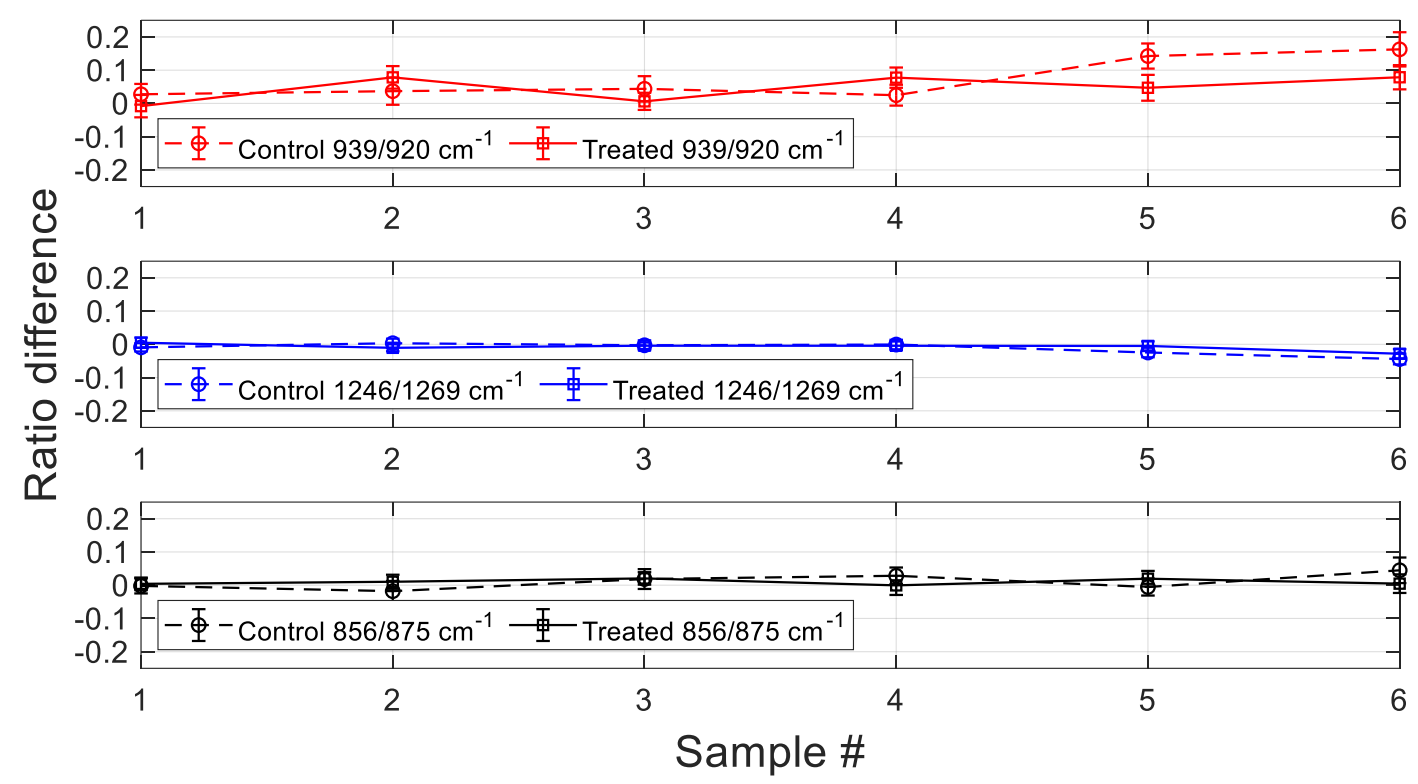

Figure A.1: Difference in intensity ratio of collagen peaks between preclear and recovered stages for treated and control samples (preclear ratio - recovered ratio). Collagen peaks ratios are related to collagen stability as described in Section 1.6. 
Appendix B: Mesh sensitivity study 
Description: A mesh sensitivity study was conducted on models representing the extremes of either tissue thickness or indentation strain. The criteria for an acceptable mesh refinement was a difference in maximum stress of no more than $5 \%$ with a doubling of element count. This criterion was selected based off of previous studies using the same limit when conducting similar FE modeling of cartilage [69]. All models used the 4200 element mesh for cartilage, with the exception of one higher strain model (treated \#5) using 5600 elements. The figure below shows the change in maximum effective stress with respect to element count for control sample \#6.

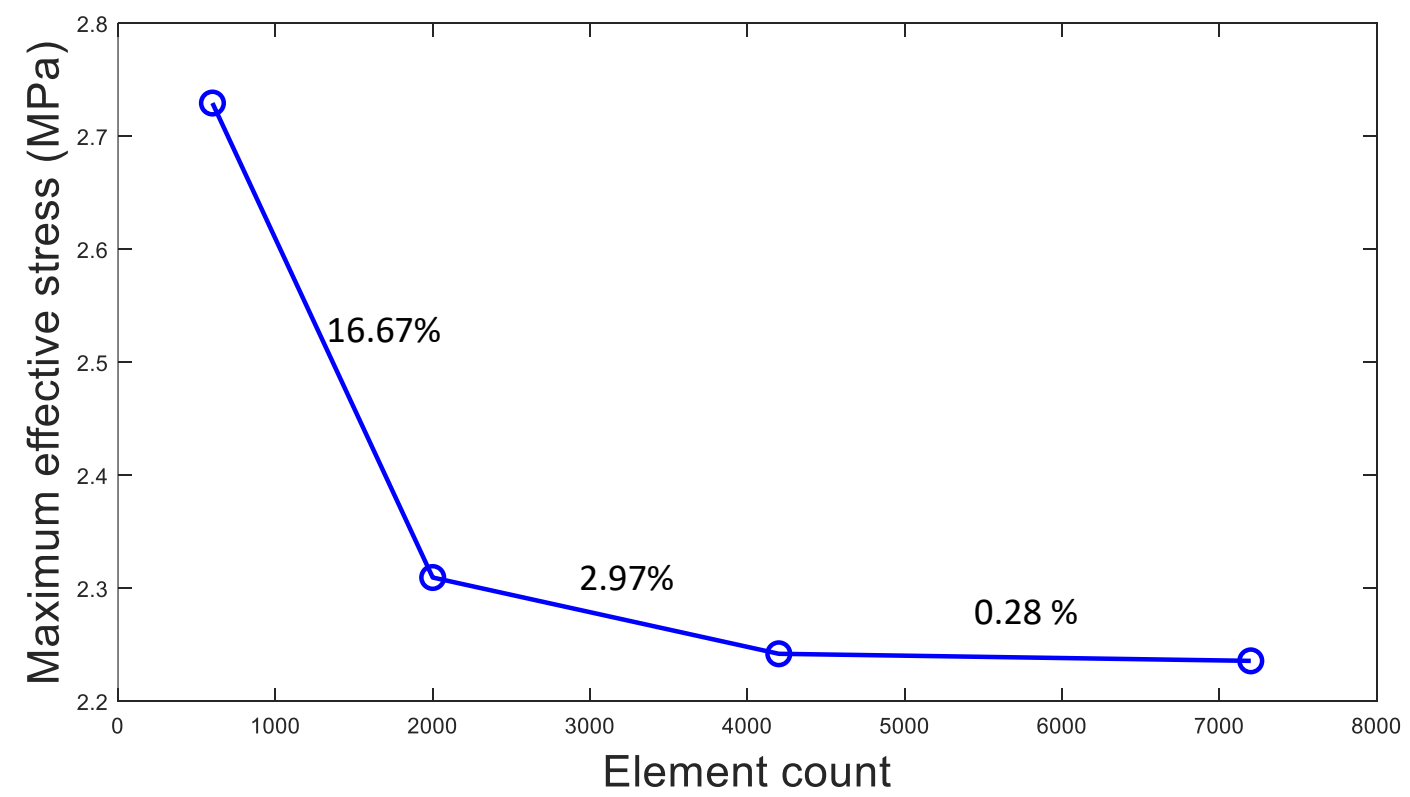

Figure B.1: Mesh sensitivity study for FE models quantifying the change in percent difference of the maximum effective stress observed in continually refined meshes. 
Appendix C: Supplemental FE model data 
Description: Supplemental data obtained from the FE models of each cartilage sample are shown in the tables below. These highlight model parameters and predicted responses in comparison to experimental results. In tables Table C.5 and Table C.6 the percent fluid load support in certain samples slightly exceeded $100 \%$; however, this was assumed to be due to numerical round off error as fluid pressure is evaluated at element nodes while the contact traction is evaluated at integration points [73].

Table C.1: Control sample FE model properties (DD = depth-dependent, $\mathrm{H}=$ homogeneous, SVF = solid volume fraction). Percent difference in objective function values represent DD models subtracted from $\mathrm{H}$ models.

\begin{tabular}{|c|c|c|c|c|c|c|}
\hline Control Sample \# & 1 & 2 & 3 & 4 & 5 & 6 \\
\hline Thickness (mm) & 1.70 & 1.90 & 1.57 & 1.79 & 1.96 & 2.10 \\
\hline$\%$ strain & 6.64 & 6.74 & 6.82 & 6.82 & 6.08 & 7.09 \\
\hline SVF & 0.22 & 0.25 & 0.23 & 0.21 & 0.20 & 0.19 \\
\hline $\begin{array}{l}\text { Objective } \\
\text { value (DD) } \cdot 1 \times 10^{-4}\end{array}$ & 0.15 & 2.77 & 0.16 & 0.53 & 1.46 & 2.03 \\
\hline E-scale & 0.15 & 0.37 & 0.12 & 0.16 & 0.50 & 0.52 \\
\hline$\xi$-scale (DD) & 0.39 & 0.96 & 0.51 & 0.78 & 0.63 & 0.78 \\
\hline $\begin{array}{l}\text { Perm (DD) } \\
1 \times 10^{-15}\left(\mathrm{~m}^{4} / \mathrm{N} \cdot \mathrm{s}\right)\end{array}$ & 27.81 & 3.27 & 22.05 & 10.01 & 5.66 & 3.16 \\
\hline $\begin{array}{l}\text { Objective } \\
\text { value }(\mathrm{H}) \cdot 1 \times 10^{-4}\end{array}$ & 0.18 & 2.51 & 0.20 & 0.66 & 1.06 & 2.10 \\
\hline E-scale (H) & 0.09 & 0.21 & 0.06 & 0.08 & 0.26 & 0.27 \\
\hline ל-scale (H) & 0.21 & 0.54 & 0.29 & 0.43 & 0.35 & 0.44 \\
\hline $\begin{array}{l}\text { Perm (H) } \\
1 \times 10^{-15}\left(\mathrm{~m}^{4} / \mathrm{N} \cdot \mathrm{s}\right)\end{array}$ & 30.25 & 3.47 & 23.21 & 10.32 & 6.03 & 3.39 \\
\hline $\begin{array}{l}\text { Objective value } \\
\% \text { difference (H-DD) }\end{array}$ & 15.33 & -9.74 & 20.49 & 22.14 & -31.56 & 2.99 \\
\hline
\end{tabular}


Table C.2: Treated sample FE model properties (DD = depth-dependent, $\mathrm{H}=$ homogeneous, SVF = solid volume fraction). Percent difference in objective function values represent DD models subtracted from $\mathrm{H}$ models.

\begin{tabular}{|c|c|c|c|c|c|}
\hline Treated Sample \# & 1 & 2 & 3 & 4 & 5 \\
\hline Thickness (mm) & 2.23 & 2.15 & 1.91 & 2.35 & 1.48 \\
\hline$\%$ strain & 7.09 & 7.21 & 7.66 & 6.59 & 9.03 \\
\hline SVF & 0.21 & 0.27 & 0.23 & 0.22 & 0.24 \\
\hline $\begin{array}{l}\text { Objective } \\
\text { value (DD) } \cdot 1 \times 10^{-4}\end{array}$ & 4.88 & 3.75 & 0.93 & 1.67 & 1.14 \\
\hline E-scale & 0.83 & 0.77 & 0.27 & 0.45 & 0.36 \\
\hline$\xi$-scale (DD) & 0.95 & 0.80 & 0.82 & 0.84 & 0.93 \\
\hline $\begin{array}{l}\text { Perm (DD) · } \\
1 \times 10^{-15}\left(\mathrm{~m}^{4} / \mathrm{N} \cdot \mathrm{s}\right)\end{array}$ & 2.39 & 3.15 & 9.21 & 5.40 & 6.79 \\
\hline $\begin{array}{l}\text { Objective } \\
\text { value }(\mathrm{H}) \cdot 1 \times 10^{-4}\end{array}$ & 5.89 & 4.41 & 1.42 & 2.03 & 2.80 \\
\hline E-scale (H) & 0.44 & 0.39 & 0.13 & 0.20 & 0.21 \\
\hline$\xi$-scale (H) & 0.56 & 0.47 & 0.45 & 0.47 & 0.33 \\
\hline $\begin{array}{l}\text { Perm (H) } \\
1 \times 10^{-15}\left(\mathrm{~m}^{4} / \mathrm{N} \cdot \mathrm{s}\right)\end{array}$ & 2.72 & 3.54 & 9.68 & 5.67 & 4.72 \\
\hline $\begin{array}{l}\text { Objective value } \\
\% \text { difference (H-DD) }\end{array}$ & 18.74 & 16.03 & 42.03 & 19.78 & 84.38 \\
\hline
\end{tabular}

Table C.3: Control sample reaction forces in preclear vs. recovered stages (RF = reaction force). Equilibrium RF represents average of last $10 \mathrm{~s}$ of $1800 \mathrm{~s}$ indentation test.

\begin{tabular}{|l|c|c|c|c|c|c|}
\hline Control Sample \# & $\mathbf{1}$ & $\mathbf{2}$ & $\mathbf{3}$ & $\mathbf{4}$ & $\mathbf{5}$ & $\mathbf{6}$ \\
\hline $\begin{array}{l}\text { Preclear peak } \\
\text { RF (mN) }\end{array}$ & $4.89 \mathrm{E}+02$ & $1.90 \mathrm{E}+03$ & $7.10 \mathrm{E}+02$ & $1.28 \mathrm{E}+03$ & $1.14 \mathrm{E}+03$ & $1.99 \mathrm{E}+03$ \\
\hline $\begin{array}{l}\text { Preclear } \\
\text { equilibrium RF (mN) }\end{array}$ & 26.06 & 88.72 & 34.92 & 53.26 & 85.21 & 127.98 \\
\hline $\begin{array}{l}\text { Recovered peak } \\
\text { RF (mN) }\end{array}$ & $5.31 \mathrm{E}+02$ & $2.02 \mathrm{E}+03$ & $6.54 \mathrm{E}+02$ & $1.22 \mathrm{E}+03$ & $1.30 \mathrm{E}+03$ & $1.92 \mathrm{E}+03$ \\
\hline $\begin{array}{l}\text { Recovered } \\
\text { equilibrium RF }(\mathbf{m N})\end{array}$ & 27.91 & 83.68 & 21.29 & 31.89 & 86.56 & 122.13 \\
\hline
\end{tabular}


Table C.4: Treated sample reaction forces in preclear vs. recovered stages (RF = reaction force). Equilibrium RF represents average of last $10 \mathrm{~s}$ of $1800 \mathrm{~s}$ indentation test.

\begin{tabular}{|l|c|c|c|c|c|}
\hline Treated Sample \# & $\mathbf{1}$ & $\mathbf{2}$ & $\mathbf{3}$ & $\mathbf{4}$ & $\mathbf{5}$ \\
\hline $\begin{array}{l}\text { Preclear peak } \\
\text { RF }(\mathbf{m N})\end{array}$ & $1.98 \mathrm{E}+03$ & $2.02 \mathrm{E}+03$ & $1.48 \mathrm{E}+03$ & $1.84 \mathrm{E}+03$ & $1.84 \mathrm{E}+03$ \\
\hline $\begin{array}{l}\text { Preclear } \\
\text { equilibrium RF }(\mathbf{m N})\end{array}$ & 152.06 & 159.92 & 69.90 & 108.32 & 81.56 \\
\hline $\begin{array}{l}\text { Recovered peak } \\
\text { RF (mN) }\end{array}$ & $2.66 \mathrm{E}+03$ & $2.22 \mathrm{E}+03$ & $1.44 \mathrm{E}+03$ & $1.73 \mathrm{E}+03$ & $1.86 \mathrm{E}+03$ \\
\hline $\begin{array}{l}\text { Recovered } \\
\text { equilibrium RF }(\mathbf{m N})\end{array}$ & 204.21 & 175.07 & 62.38 & 91.64 & 72.72 \\
\hline
\end{tabular}

Table C.5: Control sample FE model material response ( $R F=$ reaction force, $\mathrm{EXP}=$ experimental, $\mathrm{DD}=$ depth-dependent model, $\mathrm{H}=$ homogeneous model, $\mathrm{FLS}=$ fluid load support). Equilibrium RF represents average of last $10 \mathrm{~s}$ of $1800 \mathrm{~s}$ indentation test.

\begin{tabular}{|l|c|c|c|c|c|c|}
\hline Control Sample \# & $\mathbf{1}$ & $\mathbf{2}$ & $\mathbf{3}$ & $\mathbf{4}$ & $\mathbf{5}$ & $\mathbf{6}$ \\
\hline Peak RF (EXP) (mN) & $5.31 \mathrm{E}+02$ & $2.02 \mathrm{E}+03$ & $6.54 \mathrm{E}+02$ & $1.22 \mathrm{E}+03$ & $1.30 \mathrm{E}+03$ & $1.92 \mathrm{E}+03$ \\
\hline Peak RF (DD) (mN) & $4.32 \mathrm{E}+02$ & $1.66 \mathrm{E}+03$ & $5.33 \mathrm{E}+02$ & $9.82 \mathrm{E}+02$ & $1.02 \mathrm{E}+03$ & $1.62 \mathrm{E}+03$ \\
\hline Peak RF (H) (mN) & $4.23 \mathrm{E}+02$ & $1.68 \mathrm{E}+03$ & $5.37 \mathrm{E}+02$ & $9.94 \mathrm{E}+02$ & $1.04 \mathrm{E}+03$ & $1.64 \mathrm{E}+03$ \\
\hline $\begin{array}{l}\text { Equilibrium } \\
\text { RF (EXP) (mN) }\end{array}$ & 27.91 & 83.68 & 21.29 & 31.89 & 86.56 & 122.13 \\
\hline $\begin{array}{l}\text { Equilibrium } \\
\text { RF (DD) (mN) }\end{array}$ & 29.13 & 83.52 & 21.83 & 32.09 & 78.00 & 119.62 \\
\hline $\begin{array}{l}\text { Equilibrium } \\
\text { RF (H) (mN) }\end{array}$ & 28.37 & 79.35 & 20.80 & 29.61 & 83.23 & 115.20 \\
\hline $\begin{array}{l}\text { Max fluid pressure } \\
\text { (MPa) (DD) }\end{array}$ & 0.25 & 0.91 & 0.32 & 0.56 & 0.54 & 0.87 \\
\hline $\begin{array}{l}\text { Max First Principal } \\
\text { stress (MPa) (DD) }\end{array}$ & 0.56 & 8.53 & 3.32 & 3.96 & 0.44 & 1.96 \\
\hline $\begin{array}{l}\text { Max First Principal } \\
\text { strain (DD) }\end{array}$ & $5.70 \mathrm{E}-02$ & $6.13 \mathrm{E}-02$ & $5.27 \mathrm{E}-02$ & $6.20 \mathrm{E}-02$ & $5.56 \mathrm{E}-02$ & $7.11 \mathrm{E}-02$ \\
\hline Max FLS ratio (DD) & 1.05 & 1.04 & 1.06 & 1.06 & 1.03 & 1.04 \\
\hline $\begin{array}{l}\text { Max fluid pressure } \\
\text { (MPa) (H) }\end{array}$ & 0.24 & 0.89 & 0.31 & 0.57 & 0.53 & 0.83 \\
\hline $\begin{array}{l}\text { Max First Principal } \\
\text { stress (MPa) (H) }\end{array}$ & 0.20 & 0.56 & 0.26 & 0.46 & 0.33 & 0.56 \\
\hline $\begin{array}{l}\text { Max First Principal } \\
\text { strain (H) }\end{array}$ & $6.06 \mathrm{E}-02$ & $6.62 \mathrm{E}-02$ & $6.07 \mathrm{E}-02$ & $6.94 \mathrm{E}-02$ & $5.76 \mathrm{E}-02$ & $7.49 \mathrm{E}-02$ \\
\hline Max FLS ratio (H) & 1.02 & 1.03 & 1.08 & 1.04 & 1.01 & 0.95 \\
\hline
\end{tabular}


Table C.6: Treated sample FE model material response (RF = reaction force, EXP = experimental, DD = depth-dependent model, $\mathrm{H}$ = homogeneous model, $\mathrm{FLS}=$ fluid load support). Equilibrium RF represents average of last $10 \mathrm{~s}$ of $1800 \mathrm{~s}$ indentation test.

\begin{tabular}{|l|c|c|c|c|c|}
\hline Treated Sample \# & $\mathbf{1}$ & $\mathbf{2}$ & $\mathbf{3}$ & $\mathbf{4}$ & $\mathbf{5}$ \\
\hline Peak RF (EXP) (mN) & $2.66 \mathrm{E}+03$ & $2.22 \mathrm{E}+03$ & $1.44 \mathrm{E}+03$ & $1.73 \mathrm{E}+03$ & $1.86 \mathrm{E}+03$ \\
\hline Peak RF (DD) (mN) & $2.17 \mathrm{E}+03$ & $1.80 \mathrm{E}+03$ & $1.19 \mathrm{E}+03$ & $1.43 \mathrm{E}+03$ & $1.58 \mathrm{E}+03$ \\
\hline Peak RF (H) (mN) & $2.21 \mathrm{E}+03$ & $1.82 \mathrm{E}+03$ & $1.18 \mathrm{E}+03$ & $1.44 \mathrm{E}+03$ & $1.39 \mathrm{E}+03$ \\
\hline $\begin{array}{l}\text { Equilibrium } \\
\text { RF (EXP) (mN) }\end{array}$ & 204.21 & 175.07 & 62.38 & 91.64 & 72.72 \\
\hline $\begin{array}{l}\text { Equilibrium } \\
\text { RF (DD) (mN) }\end{array}$ & 199.39 & 172.47 & 59.29 & 86.89 & 73.49 \\
\hline $\begin{array}{l}\text { Equilibrium } \\
\text { RF (H) (mN) }\end{array}$ & 189.09 & 164.50 & 55.03 & 82.81 & 88.16 \\
\hline $\begin{array}{l}\text { Max fluid pressure } \\
\text { (MPa) (DD) }\end{array}$ & 1.15 & 0.94 & 0.68 & 0.77 & 0.90 \\
\hline $\begin{array}{l}\text { Max First Principal } \\
\text { stress (MPa) (DD) }\end{array}$ & 3.03 & 2.89 & 5.09 & 1.80 & 5.48 \\
\hline $\begin{array}{l}\text { Max First Principal } \\
\text { strain (DD) }\end{array}$ & $7.46 \mathrm{E}-02$ & $7.52 \mathrm{E}-02$ & $6.90 \mathrm{E}-02$ & $6.53 \mathrm{E}-02$ & $7.74 \mathrm{E}-02$ \\
\hline Max FLS ratio (DD) & 1.00 & 1.04 & 1.05 & 1.05 & 1.04 \\
\hline $\begin{array}{l}\text { Max fluid pressure } \\
\text { (MPa) (H) }\end{array}$ & 1.10 & 0.90 & 0.66 & 0.76 & 0.71 \\
\hline $\begin{array}{l}\text { Max First Principal } \\
\text { stress (MPa) (H) }\end{array}$ & 0.73 & 0.62 & 0.61 & 0.53 & 0.55 \\
\hline $\begin{array}{l}\text { Max First Principal } \\
\text { strain (H) }\end{array}$ & $7.55 \mathrm{E}-02$ & $7.59 \mathrm{E}-02$ & $8.03 \mathrm{E}-02$ & $7.09 \mathrm{E}-02$ & $9.15 \mathrm{E}-02$ \\
\hline Max FLS ratio (H) & 0.98 & 1.00 & 1.00 & 0.97 & 1.01 \\
\hline
\end{tabular}


Appendix D: Depth-dependent permeability FE models 
Description: To assess the effects of depth-dependent permeability on cartilage two preliminary models were created using the beta version of FEBioStudio 1.0.0. In these models, representing treated sample $\# 1$ and control sample \#5, the permeability was scaled proportional to the normalized water content determined from Raman spectroscopy. These models were simply meant to be a preliminary test, and not a comprehensive study on the material response.

The only means found of applying a surface-to-surface map for material properties in this beta software version involved restricting meshes to one volume with only one element type. Therefore, the standard mesh model had to be altered into one part with the wedge-shaped elements at the symmetry axis deleted. These deletions are highlighted in Figure D.1. This also meant the mesh could not be biased towards the indenter edge where maximum stresses occur. These altered models consisted of 8715 hexahedral elements while the standard model consisted of 4200 elements for the cartilage.
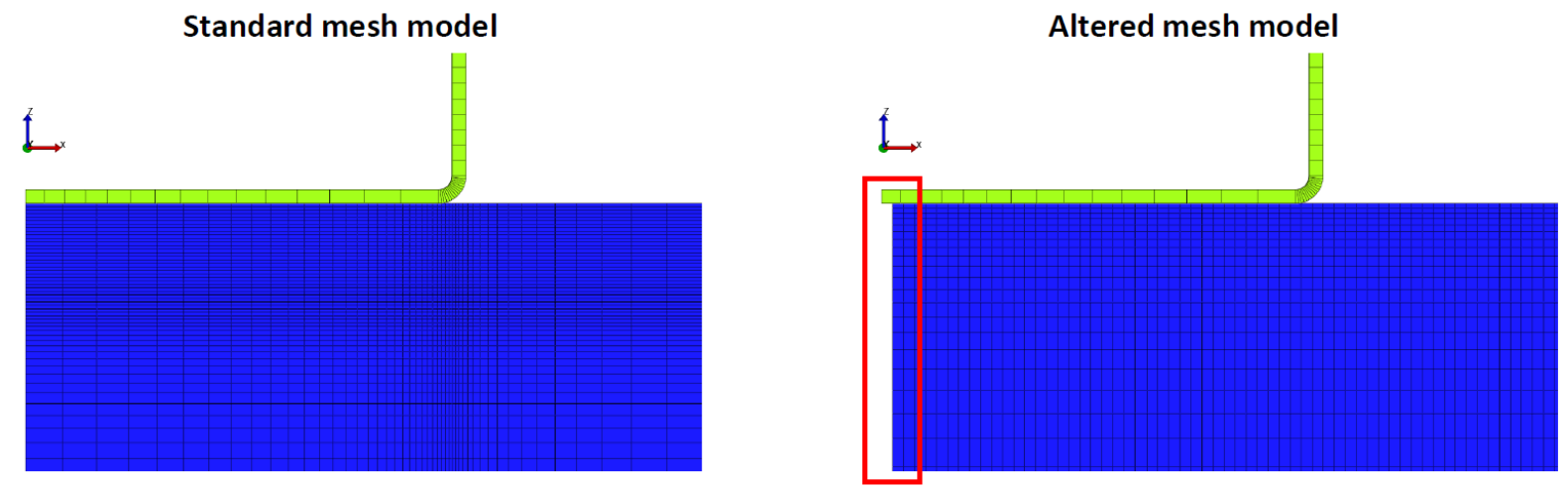

Figure D.1: Alterations to the cartilage mesh made to accommodate material property surface-tosurface maps in FEBioStudio 1.0.0. Tetrahedral element deletions are highlighted by the red square.

Three models were compared for the two samples: the standard depth-dependent model with constant permeability, the altered mesh model with the same material properties as the standard model, and the altered mesh model with depth-dependent permeability based on Raman results. These comparisons are shown below in Table D.1. The altered mesh model using constant permeability produced a very 
similar response to the standard model in treated sample \#1, but differed notably in tensile stress for control sample \#5. The tensile strain in both altered models with constant permeability was higher than their standard model counterparts. These differences are the result of element deletions and a lack of mesh refinement. Despite these differences both models produced objective function values very similar to the standard models. In addition to this the alternate mesh models with depth-dependent permeability produced nearly the same response as the altered mesh models with constant permeability. It was based on this result that depth-dependent permeability proportional to Raman results was neglected from FE models.

Table D.1: Standard and altered mesh model properties (std = standard depth-dependent model with constant permeability, alt-CP = altered mesh model with constant permeability, alt-DDP = altered mesh model with depth-dependent permeability).

\begin{tabular}{|c|c|c|c|c|c|c|}
\hline Model & $\begin{array}{c}\text { Treated } \\
\text { Sample \#1 } \\
\text { (std) }\end{array}$ & $\begin{array}{l}\text { Treated } \\
\text { Sample \#1 } \\
\text { (alt-CP) }\end{array}$ & $\begin{array}{c}\text { Treated } \\
\text { Sample \#1 } \\
\text { (alt-DDP) }\end{array}$ & $\begin{array}{l}\text { Control } \\
\text { Sample \#5 } \\
\text { (std) }\end{array}$ & $\begin{array}{l}\text { Control } \\
\text { Sample \#5 } \\
\text { (alt-CP) }\end{array}$ & $\begin{array}{c}\text { Control } \\
\text { Sample \#5 } \\
\text { (alt-DDP) }\end{array}$ \\
\hline E-scale & \multicolumn{2}{|c|}{0.83} & 0.83 & \multicolumn{2}{|c|}{0.50} & 0.51 \\
\hline ל-scale & \multicolumn{2}{|c|}{0.95} & 0.97 & \multicolumn{2}{|c|}{0.63} & 0.66 \\
\hline $\begin{array}{l}\text { Permeability } \\
1 \times 10^{-15}\left(\mathrm{~m}^{4} / \mathrm{N} \cdot \mathrm{s}\right)\end{array}$ & \multicolumn{2}{|c|}{2.39} & 2.91 & \multicolumn{2}{|c|}{5.66} & 9.16 \\
\hline $\begin{array}{l}\text { Objective } \\
\text { value } \cdot 1 \times 10^{-4}\end{array}$ & 4.88 & 4.96 & 5.01 & 1.46 & 1.46 & 1.43 \\
\hline $\begin{array}{l}\text { Max fluid } \\
\text { pressure (MPa) }\end{array}$ & 1.15 & 1.15 & 1.14 & 0.54 & 0.53 & 0.55 \\
\hline $\begin{array}{l}\text { Max First } \\
\text { Principal stress } \\
\text { (MPa) }\end{array}$ & 3.03 & 3.29 & 3.46 & 0.44 & 0.27 & 0.26 \\
\hline $\begin{array}{l}\text { Max First } \\
\text { Principal strain }\end{array}$ & 7.46E-02 & $9.18 \mathrm{E}-02$ & $9.23 \mathrm{E}-02$ & $5.56 \mathrm{E}-02$ & 7.09E-02 & $7.08 \mathrm{E}-02$ \\
\hline Max FLS ratio & 1.00 & 1.04 & 1.01 & 1.03 & 1.05 & 1.03 \\
\hline
\end{tabular}


Appendix E: Raman spectra processing scripts (pseudo code) 


\section{Description: This appendix details the main Matlab script and functions utilized in pre-processing of}

\section{Cartilage Raman spectra.}

\section{E.1 Function for removing cosmic rays from spectra}

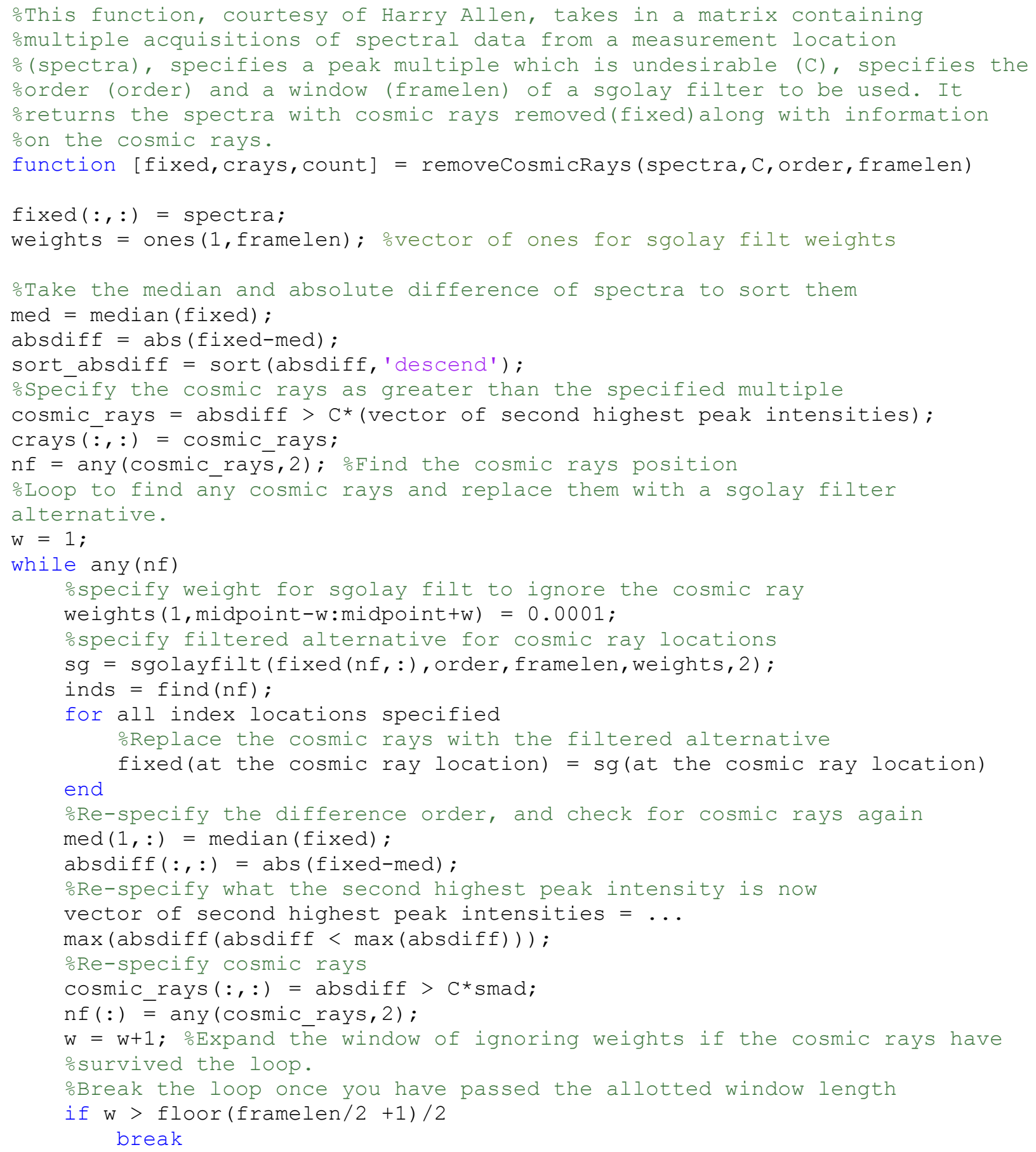




\author{
end \\ end \\ end ․ㅡㅇ of function
}

\title{
E.2 Function for removing fluorescent background from spectra
}

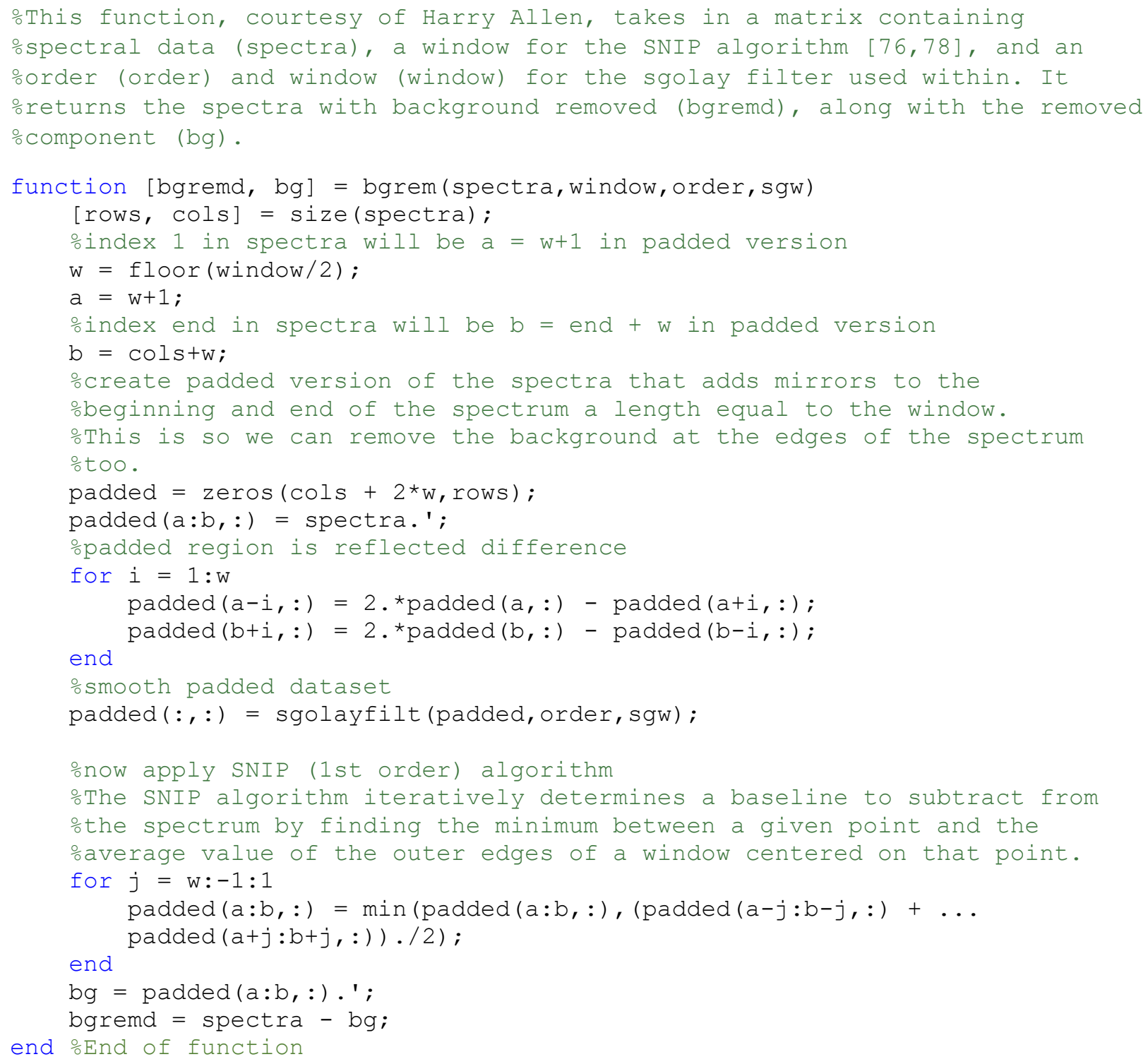

\section{E.3 Script for processing Raman spectra from cartilage}

othis script processes the raw Raman spectral data by carrying out NIST ointensity corrections, removing cosmic rays, and removing the fluorescent \%background. 
oSpecify polynomial constants and the NIST curve based on Choquette ot al. [77]. $\mathrm{a} 0=9.71937 \mathrm{E}-2 ; \mathrm{a} 1=2.28325 \mathrm{E}-4 ; \mathrm{a} 2=-5.86762 \mathrm{E}-8 ; \mathrm{a} 3=2.16023 \mathrm{E}-10 ;$ $\mathrm{a} 4=-9.77171 \mathrm{e}-14 ; \mathrm{a} 5=1.15596 \mathrm{E}-17$; polyCurve $=@(x) a 0+a{ }^{\star} x+a{ }^{\star} x \cdot \wedge 2+a 3{ }^{\star} x \cdot \wedge 3+a 4{ }^{\star} x \cdot \wedge 4+a{ }^{\star} x \cdot \wedge 5$;

\%Read in NIST file, average spectra between two measurement periods. NISTspectra = mean (two measurement periods);

ochange wavelength to wavenumber.

Wavenmr $=(((1 / 784.695)-(1 . /($ wavelength values $))) . \star 1 e 7)$;

Wavenmr $=$ set limits between wavenumber range $[400$ 1800];

Remove cosmic rays from NIST spectra specifying (spectra, multiple of peak oto remove, sgolay filter order, window).

NISTspectra $=$ removeCosmicRays (NISTspectra, $1.7,2,15)$;

ointensity correction curve to multiply spectra by is NIST polynomial curve

nist $=$ polyCurve (Wavenmr);

corrCurve = nist./NISTspectra

oThree baseline measurements are taken during measurement of cartilage from osurface to bone. These baselines are just the background intensities

orecorded when not focused on a sample.

baseline = mean ( 3 baseline measurements);

for all files containing cartilage spectral data cartilage_spectra = removeCosmicRays (cartilage_spectra, 1.7,2,15);

oSubtract baseline and multiply by correction curve for all 15 acquisitions of cartilage spectra in one file. for $J=1: 15$

cartilage_spectra $(\mathrm{J},:)=\operatorname{cartilage}$ spectra $(\mathrm{J},:)$-baseline; cartilage_spectra $(\mathrm{J},:)=$ cartilage_spectra $(\mathrm{J},:) \cdot{ }^{\star} \operatorname{corrCurve}$; oVector Normalization scheme cartilage spectra $(\mathrm{J},:)=$ bgremove (cartilage $\operatorname{spectra}(\mathrm{J},:), 135,2,15)$; cartilage_spectra $(\mathrm{J},:)=\operatorname{sgolayfilt}(\operatorname{cartilage} \operatorname{spectra}(\mathrm{J},:), 3,15)$; cartilage_spectra = cartilage_spectra - min(cartilage_spectra); surf $\operatorname{norm}(\mathrm{J}, 1)=\operatorname{norm}(\operatorname{cartilage} \operatorname{spectra}(\mathrm{J},:))$;

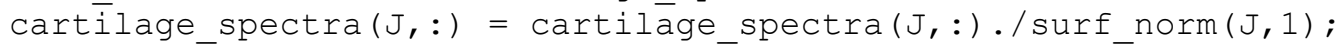
End

ofind standard deviation of each peak over 15 acquisitions. cartilage_spectra_std = std(cartilage_spectra); cartilage_spectra $=$ mean(cartilage_spectra); \%Average all spectra into one

ocarry out individual peak ratio calculations across all acquisitions to get oappropriate confidence intervals, use standard deviation data. end $\%$ End of script 
Appendix F: FEBio input files \& DataMap alterations (pseudo code) 
Description: This appendix details the alterations made to the DataMap plugin to create optimizable

scaling factors. It also shows the corresponding alterations made to the FEBio XML input files to implement the optimizable scaling factors.

\section{F.1 DataMap header file}

//The following are the alterations that must be made to the FEEFDNeoHookean2.h file to //make E and ksi optimizable from a scaling factor. Once compiled the DataMap .dll file //is linked to the FEBio configuration file.

//Within the public domain of the FEEFDNeoHookean2 subclass in the header file specify a //scaling parameter to optimize for.

class FEEFDNeoHookean2:public FEElasticMaterial

-

$\cdot$

public:

double m_E; $\quad / / !<$ Young's modulus

double m_Escale; $\quad / / !<$ Young's modulus scaling factor

double m_v; / / < Poisson's ratio

double m_ksi[3]; $\quad / / !<\mathrm{ksi}$

double m_ksiscale; $/ / !<$ Fiber modulus scaling factor

double m_beta[3]; $\quad / /$ ! $<$ beta

•

$\cdot$

\}//End

F.2 DataMap source file

//The following are the alterations that must be made to the FEEFDNeoHookean2.cpp file to //make E and ksi optimizable from a scaling factor. Once compiled the DataMap.dll file //is linked to the FEBio configuration file.

// Within the parameter list of the source file define the material parameters for the //scaling terms.

BEGIN_PARAMETER_LIST(FEEFDNeoHookean2, FEElasticMaterial)

ADD_PARAMETER(m_E, FE_PARAM_DOUBLE, "E");

ADD_PARAMETER (m_v, FE_PARAM_DOUBLE, "v");

ADD_PARAMETER(m_Escale, FE_PARAM_DOUBLE, "Escale");

ADD_PARAMETERV (m_beta, FE_PARAM_DOUBLE, 3, "beta");

ADD_PARAMETERV (m_ksi , FE_PARAM_DOUBLE, 3, "ksi" );

ADD_PARAMETER(m_ksiscale, FE_PARAM_DOUBLE, "ksiscale");

END_PARAMETER_LIST( );

•

.

• 
//Within the Stress and Tangent functions define $\mathrm{E}$ and Ksi to be scaled from the //material parameters.

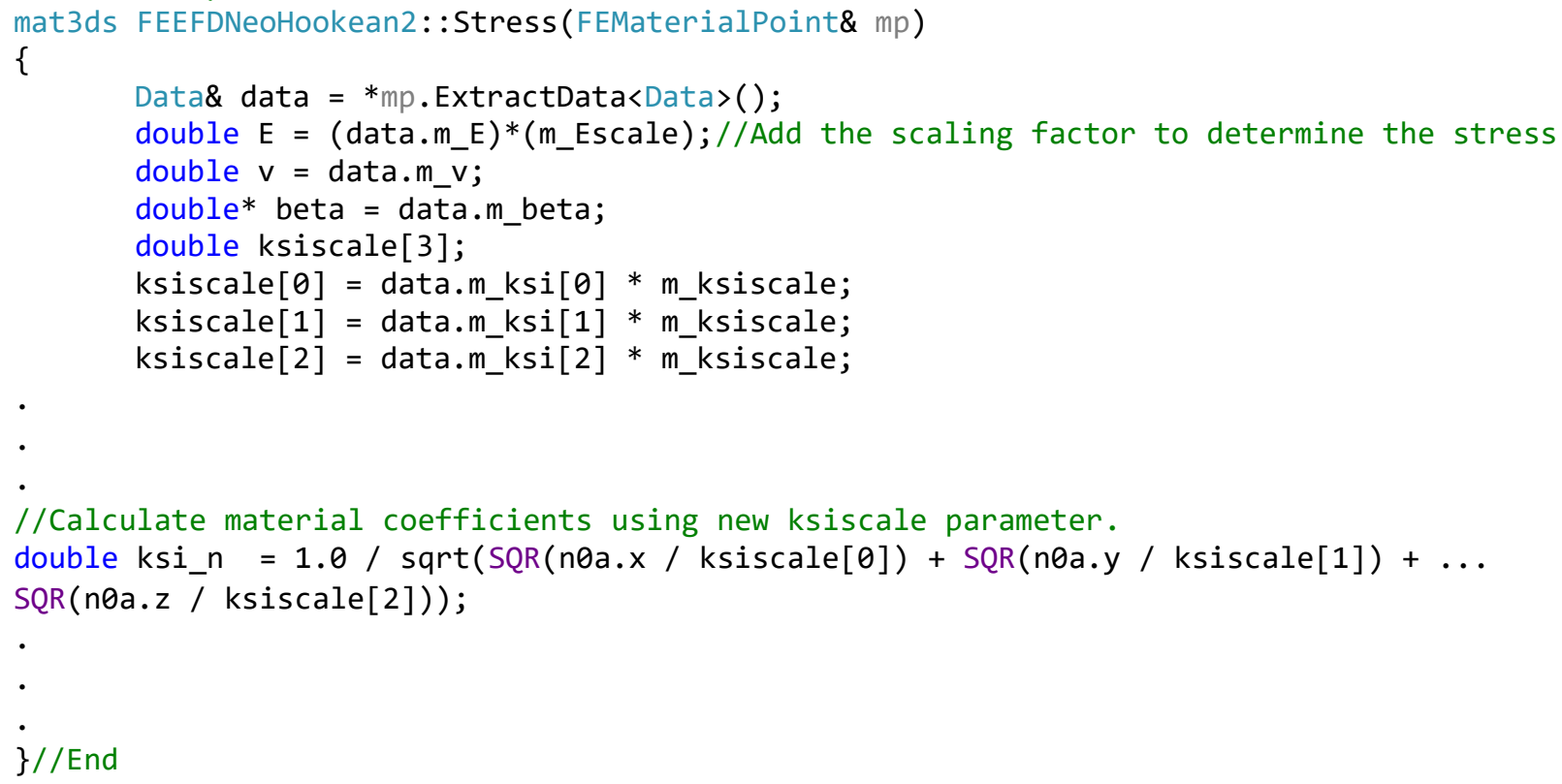

\section{F.3 FEBio XML input file}

$\%$ The following program lists only components of the XML input file of an individual sample enabling use of the \%altered DataMap plugin. These alterations are sample specific based on Raman results. This data is fed into the \%command line along with an optimization script to find the ideal material parameters for the sample.

$\%$ Define $\mathrm{xml}$ version and control parameters.

$<$ ?xml version="1.0" encoding="ISO-8859-1"?>

$<$ febio_spec version="2.5">

$<$ Module type="biphasic"/>

$<$ Control>

$\%$ Set the time steps to be a multiple of the indentation test.

$$
\begin{aligned}
& \text { <time_steps }>18021<\text { /time_steps }> \\
& <\text { step_size }>0.1<\text { /step_size }> \\
& \text { <max_refs }>30<\text { /max_refs }> \\
& <\text { max_ups }>15<\text { /max_ups }>
\end{aligned}
$$

$<$ max_retries $>30</$ max_retries $>$

$<$ opt_iter $>10</ o p t \_i t e r>$

$</$ time_stepper>

$<$ symmetric_biphasic $>0</$ symmetric_biphasic $>$

\section{$</$ Control $>$}

$\%$ Define material to be used. Two rigid bodies, and a biphasic material for the cartilage. The cartilage material is \%based on the altered Neohookean2 material from DataMap.

$<$ Material> 
<material id="3" name="Cartilage" type="biphasic">

$\%$ SVF based on wet and dry weights with the calculated volume.

$$
\begin{aligned}
& <\text { phi0>0.2064</phi0> } \\
& <\text { fluid_density>1</fluid_density> } \\
& <\text { solid type="EFD neo-Hookean2"> }
\end{aligned}
$$

$\%$ Scaling parameters and permeability based on optimized results. Set E and ksi to be equal to unity so the scaling $\%$ factors are represented proportionally.

$<$ Escale $>0.8293512559393588</$ Escale $>$

$<$ ksiscale $>0.9521160943065482</$ ksiscale $>$

$<\mathrm{E}>1.0</ \mathrm{E}>$

$<\mathrm{v}>0.03</ \mathrm{v}>$

$<$ beta $>2.0,2.0,2.0</$ beta $>$

$<$ ksi $>1 \cdot 0,1 \cdot 0,1 \cdot 0</ \mathrm{ksi}>$

$<$ solid $>$

<permeability type="perm-const-iso">

$<$ perm>0.002392949379621985</perm>

$</$ permeability $>$

$</$ material $>$

$</$ Material $>$

$\%$ Specify loadcurves for the files after creating node and part information $<$ LoadData>

$\% 1 c$ for must points.

$$
\begin{aligned}
& \text { <loadcurve id="1" type="linear"> } \\
& \quad<\text { point }>0,0</ \text { point }> \\
& \quad<\text { point }>4.868,0.05</ \text { point }> \\
& \quad<\text { point }>120.622,5</ \text { point }> \\
& \quad<\text { point }>1802.04,50</ \text { point }> \\
& \text { </loadcurve }>
\end{aligned}
$$

$\%$ lc for indenter displacement.

$<$ loadcurve id="2" type="linear">

$<$ point $>0,0</$ point $>$

$<$ point $>0.179,-0.006</$ point $>$

$<$ point $>0.271,-0.009</$ point $>$

\section{$</$ loadcurve $>$}

\%1c for surface-to-surface map of E based on normalized Raman GAG content.

$<$ loadcurve id="3" type="linear">

$<$ point $>0,0.9469</$ point $>$

$<$ point $>0.2392,0.9469</$ point $>$

$<$ point $>0.4928,1.0<$ point $>$

$<$ point $>0.7464,0.7869</$ point $>$

$<$ point $>0.9551,0.3998</$ point $>$

$<$ point $>1.0,0.0891</$ point $>$

$</$ loadcurve $>$ 


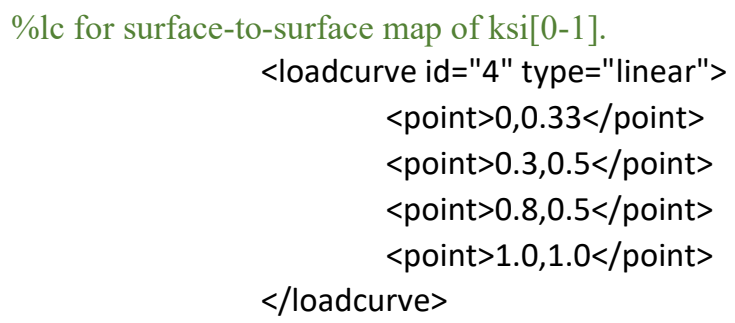

$\%$ Specify parts for surface-to-surface map application

<ElementData generator="surface-to-surface map" var="E" elem_set="Part10"> <bottom_surface>bottom</bottom_surface> $<$ top_surface $>$ top $</$ top_surface $>$ $<$ function Ic="3" $>1</$ function $>$

$</$ MeshData $>$

$<$ ElementData>

$<$ Boundary $>$

$<$ Boundary $>$

$\%$ Specify the symmetry plane and biphasic contacts $<$ Contact $>$

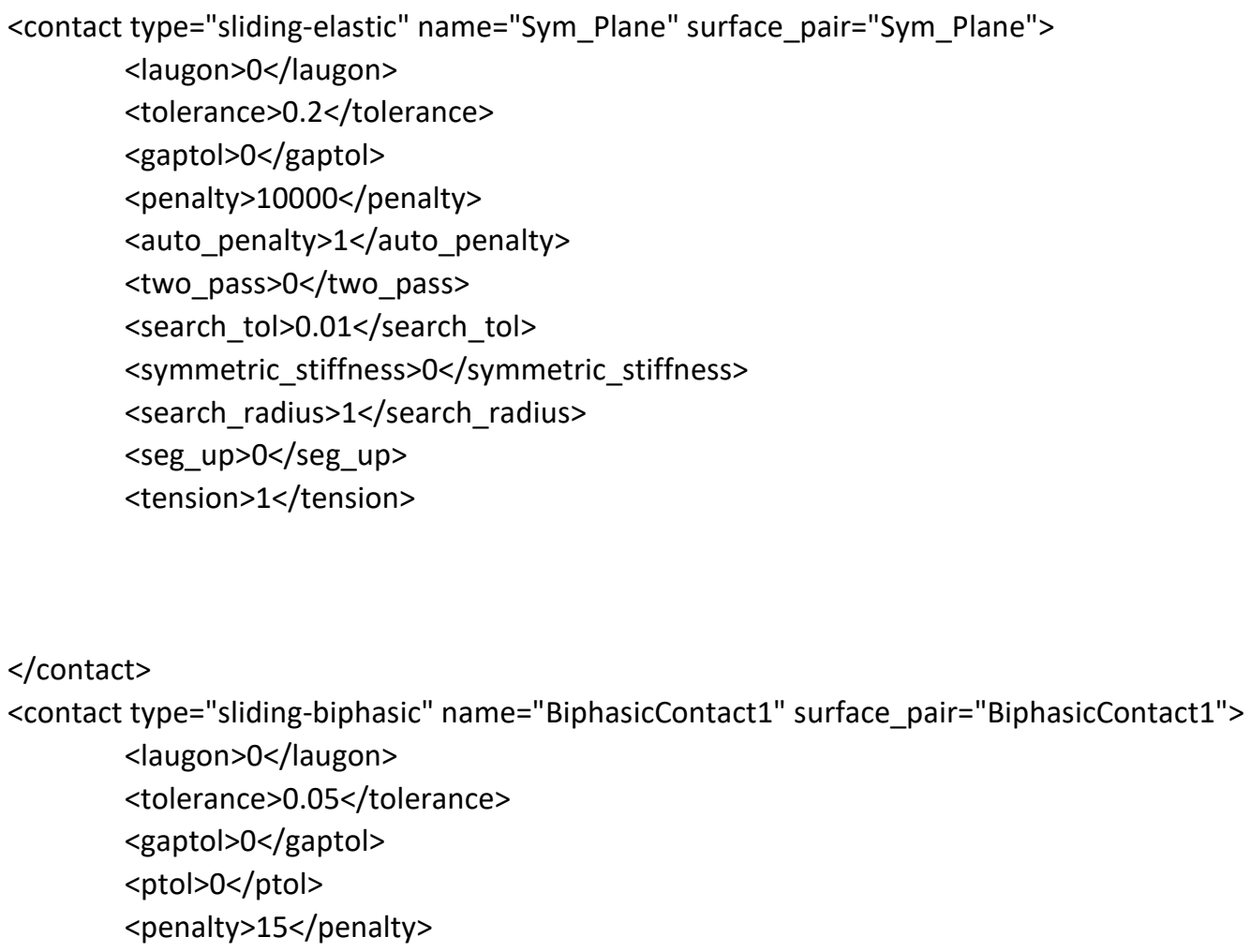




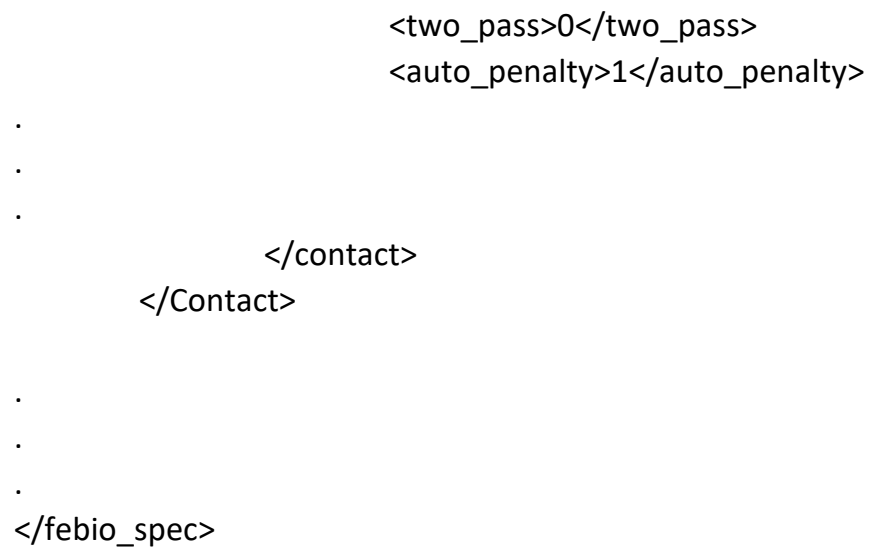

\section{F.4 FEBio XML optimization file}

\% This optimization XML file specifies the material parameters to optimize alongside the indenter reaction force $\%$ recorded during testing. The script finds the material parameters that create a model reaction force best matching $\%$ the experimental input.

$\%$ Specify the optimization version and tolerances.

$<$ ?xml version="1.0"?>

$<$ febio_optimize version="2.0">

$<$ Options>

$<$ obj_tol>1e-6</obj_tol>

$<f$ diff_scale $>1 \mathrm{e}-4</$ f_diff_scale $>$

$</$ Options $>$

$\%$ Specify the optimization parameter boundaries [initial estimate, lower bound, upper bound].

<Parameters>

$<$ param name="fem.material('Cartilage.solid').Escale" $>0.8,0.1,1.5</$ param $>$

$<$ param name="fem.material('Cartilage.solid').ksiscale" $>0.9,0.01,1.5<$ param $>$

$<$ param name="fem.material('Cartilage'). permeability.perm">0.002, 0.0001, 0.01</param>

$<$ Parameters $>$

$\%$ Specify the objective type and function.

$<$ Objective type="data-fit">

$<$ fnc type="parameter" $>$

$<$ param name="fem.rigidbody('Indenter').Fz"/>

$</$ fnc $>$

$<$ data $>$

$\%$ Input the reaction force data for the indenter from stress-relaxation tests.

$</$ data $>$

$<$ /Objective $>$

$</$ febio_optimize $>$ 